\title{
RAMANUJAN'S THEORIES OF ELLIPTIC FUNCTIONS TO ALTERNATIVE BASES
}

\author{
BRUCE C. BERNDT, S. BHARGAVA, AND FRANK G. GARVAN
}

\begin{abstract}
In his famous paper on modular equations and approximations to $\pi$, Ramanujan offers several series representations for $1 / \pi$, which he claims are derived from "corresponding theories" in which the classical base $q$ is replaced by one of three other bases. The formulas for $1 / \pi$ were only recently proved by J. M. and P. B. Borwein in 1987, but these "corresponding theories" have never been heretofore developed. However, on six pages of his notebooks, Ramanujan gives approximately 50 results without proofs in these theories. The purpose of this paper is to prove all of these claims, and several further results are established as well.
\end{abstract}

\section{INTRODUCTION}

In his famous paper [33], [36, pp. 23-39], Ramanujan offers several beautiful series representations for $1 / \pi$. He first states three formulas, one of which is

$$
\frac{4}{\pi}=\sum_{n=0}^{\infty} \frac{(6 n+1)\left(\frac{1}{2}\right)_{n}^{3}}{(n !)^{3} 4^{n}}
$$

where $(a)_{0}=1$ and, for each positive integer $n$,

$$
(a)_{n}=a(a+1)(a+2) \cdots(a+n-1) .
$$

He then remarks that "There are corresponding theories in which $q$ is replaced by one or other of the functions

$$
\begin{gathered}
q_{1}=\exp \left(-\pi \sqrt{2} K_{1}^{\prime} / K_{1}\right), \quad q_{2}=\exp \left(-2 \pi K_{2}^{\prime} /\left(K_{2} \sqrt{3}\right)\right), \quad \text { and } \\
q_{3}=\exp \left(-2 \pi K_{3}^{\prime} / K_{3}\right),
\end{gathered}
$$

where

$$
\begin{aligned}
& K_{1}={ }_{2} F_{1}\left(\frac{1}{4}, \frac{3}{4} ; 1 ; k^{2}\right), \\
& K_{2}={ }_{2} F_{1}\left(\frac{1}{3}, \frac{2}{3} ; 1 ; k^{2}\right),
\end{aligned}
$$

and

Received by the editors April 12, 1994.

1991 Mathematics Subject Classification. Primary 33E05, 33D10, 33C05, 11 F27.

Key words and phrases. $\pi$, elliptic functions, theta-functions, ordinary hypergeometric functions, elliptic integrals, modular equations, Eisenstein series, the Borweins' cubic theta-functions, principle of triplication. 


$$
K_{3}={ }_{2} F_{1}\left(\frac{1}{6}, \frac{5}{6} ; 1 ; k^{2}\right) . "
$$

Here $K_{j}^{\prime}=K_{j}\left(k^{\prime}\right)$, where $1 \leq j \leq 3, \quad k^{\prime}=\sqrt{1-k^{2}}$, and $0<k<1 ; \quad k$ is called the modulus. In the classical theory, the hypergeometric functions above are replaced by ${ }_{2} F_{1}\left(\frac{1}{2}, \frac{1}{2} ; 1 ; k^{2}\right)$. Ramanujan then offers 16 further formulas for $1 / \pi$ that arise from these alternative theories, but he provides no details for his proofs. In an appendix at the end of Ramanujan's Collected Papers, [36, p. 336], the editors, quoting L.J. Mordell, lament "It is unfortunate that Ramanujan has not developed in detail the corresponding theories referred to in $914 . "$

Ramanujan's formulas for $1 / \pi$ were not established until 1987 , when they were first proved by J.M. and P.B. Borwein [13], [14], [12, pp. 177-188]. To prove these formulas, they needed to develop only a very small portion of the "corresponding theories" to which Ramanujan alluded. In particular, the main ingredients in their work are Clausen's formula and identities relating ${ }_{2} F_{1}\left(\frac{1}{2}, \frac{1}{2} ; 1 ; x\right)$, to each of the functions ${ }_{2} F_{1}\left(\frac{1}{4}, \frac{3}{4} ; 1 ; x\right){ }_{2} F_{1}\left(\frac{1}{3}, \frac{2}{3} ; 1 ; x\right)$, and ${ }_{2} F_{1}\left(\frac{1}{6}, \frac{5}{6} ; 1 ; x\right)$. The Borweins [15], [17], further developed their ideas by deriving several additional formulas for $1 / \pi$. Ramanujan's ideas were also greatly extended by D.V. and G.V. Chudnovsky [20] who showed that other transcendental constants could be represented by similar series and that an infinite class of such formulas existed.

Ramanujan's "corresponding theories" have not been heretofore developed. Initial steps were taken by K. Venkatachaliengar [41, pp. 89-95] who examined some of the entries in Ramanujan's notebooks [35] devoted to his alternative theories.

The greatest advances toward establishing Ramanujan's theories have been made by J.M. and P.B. Borwein [16]. In searching for analogues of the classical arithmetic-geometric mean of Gauss, they discovered an elegant cubic analogue. Playing a central role in their work is a cubic transformation formula for ${ }_{2} F_{1}\left(\frac{1}{3}, \frac{2}{3} ; 1 ; x\right)$, which, in fact, is found on page 258 of Ramanujan's second notebook [35], and which was rediscovered by the Borweins. A third major discovery by the Borweins is a beautiful and surprising cubic analogue of a famous theta-function identity of Jacobi for fourth powers. We shall describe these findings in more detail in the sequel.

As alluded in the foregoing paragraphs, Ramanujan had recorded some results in his three alternative theories in his second notebook [35]. In fact, six pages, pp. 257-262, are devoted to these theories. These are the first six pages in the 100 unorganized pages of material that immediately follow the 21 organized chapters in the second notebook. Our objective in this paper is to establish all of these claims. In proving these results, it is very clear to us that Ramanujan had established further results that he unfortunately did not record either in his notebooks and other unpublished papers or in his published papers. Moreover, Ramanujan's work points the way to many additional theorems in these theories, and we hope that others will continue to develop Ramanujan's beautiful ideas.

The most important of the three alternative theories is the one arising from the hypergeometric function ${ }_{2} F_{1}\left(\frac{1}{3}, \frac{2}{3} ; 1 ; x\right)$. The theories in the remaining two cases are more easily extracted from the classical theory and so are of less interest. 
We first review the terminology in the relevant classical theory of elliptic functions, which, for example, can be found in Whittaker and Watson's text [42]. However, since we utilize many results in the classical theory that were initially found and recorded by Ramanujan in his notebooks [35], we employ much of his notation.

The complete elliptic integral of the first kind $K=K(k)$ associated with the modulus $k, 0<k<1$, is defined by

$$
K:=\int_{0}^{\pi / 2} \frac{d \phi}{\sqrt{1-k^{2} \sin ^{2} \phi}}=\frac{\pi}{2}{ }_{2} F_{1}\left(\frac{1}{2}, \frac{1}{2} ; 1 ; k^{2}\right),
$$

where the latter representation is achieved by expanding the integrand in a binomial series and integrating termwise. For brevity, Ramanujan sets

$$
z:=\frac{2}{\pi} K={ }_{2} F_{1}\left(\frac{1}{2}, \frac{1}{2} ; 1 ; k^{2}\right) .
$$

The base (or nome) $q$ is defined by

$$
q:=e^{-\pi K^{\prime} / K}
$$

where $K^{\prime}=K\left(k^{\prime}\right)$. Ramanujan sets $x \quad($ or $\alpha)=k^{2}$.

Let $n$ denote a fixed positive integer, and suppose that

$$
n \frac{{ }_{2} F_{1}\left(\frac{1}{2}, \frac{1}{2} ; 1 ; 1-k^{2}\right)}{{ }_{2} F_{1}\left(\frac{1}{2}, \frac{1}{2} ; 1 ; k^{2}\right)}=\frac{{ }_{2} F_{1}\left(\frac{1}{2}, \frac{1}{2} ; 1 ; 1-\ell^{2}\right)}{{ }_{2} F_{1}\left(\frac{1}{2}, \frac{1}{2} ; 1 ; \ell^{2}\right)},
$$

where $0<k, \ell<1$. Then a modular equation of degree $n$ is a relation between the moduli $k$ and $\ell$ which is implied by (1.4). Following Ramanujan, we put $\alpha=k^{2}$ and $\beta=\ell^{2}$. We often say that $\beta$ has degree $n$, or degree $n$ over $\alpha$. The multiplier $m$ is defined by

$$
m=\frac{{ }_{2} F_{1}\left(\frac{1}{2}, \frac{1}{2} ; 1 ; \alpha\right)}{{ }_{2} F_{1}\left(\frac{1}{2}, \frac{1}{2} ; 1 ; \beta\right)} .
$$

We employ analogous notation for the three alternative systems. The classical terminology described above is represented by the case $r=2$ below. For $r=2,3,4,6$ and $0<x<1$, set

$$
z(r):=z(r ; x):={ }_{2} F_{1}\left(\frac{1}{r}, \frac{r-1}{r} ; 1 ; x\right)
$$

and

In particular,

$$
q_{r}:=q_{r}(x):=\exp \left(-\pi \csc (\pi / r) \frac{{ }_{2} F_{1}\left(\frac{1}{r}, \frac{r-1}{r} ; 1 ; 1-x\right)}{{ }_{2} F_{1}\left(\frac{1}{r}, \frac{r-1}{r} ; 1 ; x\right)}\right) .
$$

$$
\begin{aligned}
& q_{3}=\exp \left(-\frac{2 \pi}{\sqrt{3}} \frac{{ }_{2} F_{1}\left(\frac{1}{3}, \frac{2}{3} ; 1 ; 1-x\right)}{{ }_{2} F_{1}\left(\frac{1}{3}, \frac{2}{3} ; 1 ; x\right)}\right), \\
& q_{4}=\exp \left(-\pi \sqrt{2} \frac{{ }_{2} F_{1}\left(\frac{1}{4}, \frac{3}{4} ; 1 ; 1-x\right)}{{ }_{2} F_{1}\left(\frac{1}{4}, \frac{3}{4} ; 1 ; x\right)}\right),
\end{aligned}
$$

and 


$$
q_{6}=\exp \left(-2 \pi \frac{{ }_{2} F_{1}\left(\frac{1}{6}, \frac{5}{6} ; 1 ; 1-x\right)}{{ }_{2} F_{1}\left(\frac{1}{6}, \frac{5}{6} ; 1 ; x\right)}\right) \text {. }
$$

(We consider the notation (1.7)-(1.9) to be more natural than that of Ramanujan quoted at the beginning of this paper.)

Let $n$ denote a fixed natural number, and assume that

$$
n \frac{{ }_{2} F_{1}\left(\frac{1}{r}, \frac{r-1}{r} ; 1 ; 1-\alpha\right)}{{ }_{2} F_{1}\left(\frac{1}{r}, \frac{r-1}{r} ; 1 ; \alpha\right)}=\frac{{ }_{2} F_{1}\left(\frac{1}{r}, \frac{r-1}{r} ; 1 ; 1-\beta\right)}{{ }_{2} F_{1}\left(\frac{1}{r}, \frac{r-1}{r} ; 1 ; \beta\right)},
$$

where $r=2,3,4$, or 6 . Then a modular equation of degree $n$ is a relation between $\alpha$ and $\beta$ induced by (1.10). The multiplier $m(r)$ is defined by

$$
m(r)=\frac{{ }_{2} F_{1}\left(\frac{1}{r}, \frac{r-1}{r} ; 1 ; \alpha\right)}{{ }_{2} F_{1}\left(\frac{1}{r}, \frac{r-1}{r} ; 1 ; \beta\right)},
$$

for $r=2,3,4$, or 6 . When the context is clear, we omit the argument $r$ in $q_{r}, z(r)$, and $m(r)$.

In the sequel, we say that these theories are of signature $2,3,4$, and 6 , respectively.

Theta-functions are at the focal point in Ramanujan's theories. His general theta-function $f(a, b)$ is defined by

$$
f(a, b):=\sum_{n=-\infty}^{\infty} a^{n(n+1) / 2} b^{n(n-1) / 2}, \quad|a b|<1 .
$$

If we set $a=q e^{2 i z}, b=q e^{-2 i z}$, and $q=e^{\pi i \tau}$, where $z$ is an arbitrary complex number and $\operatorname{Im}(\tau)>0$, then $f(a, b)=\vartheta_{3}(z, \tau)$, in the classical notation of Whittaker and Watson [42, p. 464]. In particular, we utilize three special cases of $f(a, b)$, namely,

$$
\begin{aligned}
& \varphi(q):=f(q, q)=\sum_{n=-\infty}^{\infty} q^{n^{2}}, \\
& \psi(q):=f\left(q, q^{3}\right)=\sum_{n=0}^{\infty} q^{n(n+1) / 2}
\end{aligned}
$$

and

$$
f(-q):=f\left(-q,-q^{2}\right)=\sum_{n=-\infty}^{\infty}(-1)^{n} q^{n(3 n+1) / 2}=\prod_{n=1}^{\infty}\left(1-q^{n}\right),
$$

where $|q|<1$.

One of the fundamental results in the theory of elliptic functions is the inversion formula $[42$, p. 500$],[5$, p. 101 , eq. (6.4)]

$$
z={ }_{2} F_{1}\left(\frac{1}{2}, \frac{1}{2} ; 1 ; x\right)=\varphi^{2}(q) \text {. }
$$

We set

$$
z_{n}=\varphi^{2}\left(q^{n}\right),
$$

for each positive integer $n$, so that $z_{1}=z$. Thus, by (1.5), (1.15), and (1.16),

$$
m=\frac{z_{1}}{z_{n}}=\frac{\varphi^{2}(q)}{\varphi^{2}\left(q^{n}\right)} \text {. }
$$


In the sequel, unattended page numbers, particularly after the statements of theorems, refer to the pagination of the Tata Institute's publication of Ramanujan's second notebook [35]. We employ many results from Ramanujan's second notebook in our proofs, in particular, from Chapters 17, 19, 20, and 21. Proofs of all of the theorems from Chapters 16-21 of Ramanujan's second notebook can be found in [5].

2. RAMANUJAN's CUBIC TRANSFORMATION, THE BORWEINS' CUBIC THETA-FUNCTION IDENTITY, AND THE INVERSION FORMULA

In classical notation, the identity

$$
\vartheta_{3}^{4}(q)=\vartheta_{4}^{4}(q)+\vartheta_{2}^{4}(q)
$$

is Jacobi's famous identity for fourth powers of theta-functions. In Ramanujan's notation (1.12) and (1.13), this identity has the form [35], [5, Chapter 16, Entry 25(vii)]

$$
\varphi^{4}(q)=\varphi^{4}(-q)+16 q \psi^{4}\left(q^{2}\right)
$$

J.M. and P.B. Borwein [16] discovered an elegant cubic analogue which we now relate. For $\omega=\exp (2 \pi i / 3)$, let

$$
\begin{aligned}
& a(q):=\sum_{m, n=-\infty}^{\infty} q^{m^{2}+m n+n^{2}}, \\
& b(q):=\sum_{m, n=-\infty}^{\infty} \omega^{m-n} q^{m^{2}+m n+n^{2}},
\end{aligned}
$$

and

$$
c(q):=\sum_{m, n=-\infty}^{\infty} q^{(m+1 / 3)^{2}+(m+1 / 3)(n+1 / 3)+(n+1 / 3)^{2}} .
$$

Then the Borweins [16] proved that

$$
a^{3}(q)=b^{3}(q)+c^{3}(q)
$$

They also established the alternative representations

$$
a(q)=1+6 \sum_{n=0}^{\infty}\left(\frac{q^{3 n+1}}{1-q^{3 n+1}}-\frac{q^{3 n+2}}{1-q^{3 n+2}}\right)
$$

and

$$
a(q)=\varphi(q) \varphi\left(q^{3}\right)+4 q \psi\left(q^{2}\right) \psi\left(q^{6}\right) .
$$

Formula (2.6) can also be found in one of Ramanujan's letters to Hardy, written from the nursing home, Fitzroy House [37, p. 93], and is proved by one of us in [6]. The identity (2.7) is found on page 328 in the unorganized portions of Ramanujan's second notebook [35], [8, Chapter 25, Entry 27] and was proved by one of us [5, Chapter 21, eq. (3.6)] in the course of proving some related identities in Section 3 of Chapter 21 in Ramanujan's second notebook [35]. Furthermore, the Borweins [16] proved that

$$
b(q)=\frac{1}{2}\left\{3 a\left(q^{3}\right)-a(q)\right\}
$$

and 


$$
c(q)=\frac{1}{2}\left\{a\left(q^{\frac{1}{3}}\right)-a(q)\right\} .
$$

The Borweins' proof of (2.5) employs the theory of modular forms on the group generated by the transformations $t \rightarrow 1 / t$ and $t \rightarrow t+i \sqrt{3}$. Shortly thereafter, J.M. Borwein, P.B. Borwein, and F. Garvan [18] gave a simpler, more elementary proof that does not depend upon the theory of modular forms. Although Ramanujan does not state (2.5) in his notebooks, we shall show that (2.5) may be simply derived from results given by him in his notebooks. Our proof also does not utilize the theory of modular forms.

We first establish parametric representations for $a(q), b(q)$, and $c(q)$.

Lemma 2.1. Let $m=z_{1} / z_{3}$, as in (1.17). Then

$$
\begin{aligned}
& a(q)=\sqrt{z_{1} z_{3}} \frac{m^{2}+6 m-3}{4 m}, \\
& b(q)=\sqrt{z_{1} z_{3}} \frac{(3-m)\left(9-m^{2}\right)^{\frac{1}{3}}}{4 m^{\frac{2}{3}}},
\end{aligned}
$$

and

$$
c(q)=\sqrt{z_{1} z_{3}} \frac{3(m+1)\left(m^{2}-1\right)^{\frac{1}{3}}}{4 m} .
$$

Proof. From Entry 11(iii) of Chapter 17 in Ramanujan's second notebook [35], [5, p. 123],

$$
\psi\left(q^{2}\right)=\frac{1}{2} \sqrt{z_{1}}(\alpha / q)^{\frac{1}{4}} \quad \text { and } \quad \psi\left(q^{6}\right)=\frac{1}{2} \sqrt{z_{3}}\left(\beta / q^{3}\right)^{\frac{1}{4}},
$$

where $\beta$ has degree 3 over $\alpha$. In proving Ramanujan's modular equations of degree 3 in Section 5 of Chapter 19 of Ramanujan's second notebook, Berndt [5, p. 233, eq. (5.2)] derived the parametric representations

$$
\alpha=\frac{(m-1)(3+m)^{3}}{16 m^{3}}
$$

and

$$
\beta=\frac{(m-1)^{3}(3+m)}{16 m} .
$$

Thus, by (1.16), (2.7), (2.13), (2.14), and (2.15),

$$
\begin{aligned}
a(q) & =\sqrt{z_{1} z_{3}}\left\{1+(\alpha \beta)^{\frac{1}{4}}\right\} \\
& =\sqrt{z_{1} z_{3}}\left\{1+\frac{(m-1)(m+3)}{4 m}\right\}=\sqrt{z_{1} z_{3}} \frac{m^{2}+6 m-3}{4 m},
\end{aligned}
$$

and so (2.10) is established. (In fact, (2.10) is proved in [5, p. 462, eq. (3.5)].)

Next, from (2.7) and (2.8),

$$
2 b(q)=\varphi(q) \varphi\left(q^{3}\right)\left(3 \frac{\varphi\left(q^{9}\right)}{\varphi(q)}-1\right)-4 q \psi\left(q^{2}\right) \psi\left(q^{6}\right)\left(1-3 q^{2} \frac{\psi\left(q^{18}\right)}{\psi\left(q^{2}\right)}\right)
$$

and, from (2.7) and (2.9),

$$
2 c(q)=\varphi(q) \varphi\left(q^{3}\right)\left(\frac{\varphi\left(q^{\frac{1}{3}}\right)}{\varphi\left(q^{3}\right)}-1\right)-4 q \psi\left(q^{2}\right) \psi\left(q^{6}\right)\left(1-\frac{\psi\left(q^{\frac{2}{3}}\right)}{q^{\frac{2}{3}} \psi\left(q^{6}\right)}\right) .
$$


By Entry 1(iii) of Chapter 20 [35], [5, p. 345], (1.16), and (1.17),

$$
3 \frac{\varphi\left(q^{9}\right)}{\varphi(q)}-1=\left(9 \frac{\varphi^{4}\left(q^{3}\right)}{\varphi^{4}(q)}-1\right)^{\frac{1}{3}}=\left(\frac{9}{m^{2}}-1\right)^{\frac{1}{3}}
$$

and

$$
\frac{\varphi\left(q^{\frac{1}{3}}\right)}{\varphi\left(q^{3}\right)}-1=\left(\frac{\varphi^{4}(q)}{\varphi^{4}\left(q^{3}\right)}-1\right)^{\frac{1}{3}}=\left(m^{2}-1\right)^{\frac{1}{3}} .
$$

By Entry 1(ii) in Chapter 20 [35], [5, p. 345] and (2.13)-(2.15),

$$
\begin{aligned}
1-3 q^{2} \frac{\psi\left(q^{18}\right)}{\psi\left(q^{2}\right)} & =\left(1-9 q^{2} \frac{\psi^{4}\left(q^{6}\right)}{\psi^{4}\left(q^{2}\right)}\right)^{\frac{1}{3}} \\
& =\left(1-\frac{9}{m^{2}} \frac{\beta}{\alpha}\right)^{\frac{1}{3}}=2 \frac{m^{\frac{1}{3}}(3-m)^{\frac{1}{3}}}{(m+3)^{\frac{2}{3}}}
\end{aligned}
$$

and

$$
\begin{aligned}
1-\frac{\psi\left(q^{\frac{2}{3}}\right)}{q^{\frac{2}{3}} \psi\left(q^{6}\right)} & =\left(1-\frac{\psi^{4}\left(q^{2}\right)}{q^{2} \psi^{4}\left(q^{6}\right)}\right)^{\frac{1}{3}} \\
& =\left(1-m^{2} \frac{\alpha}{\beta}\right)^{\frac{1}{3}}=-2 \frac{(m+1)^{\frac{1}{3}}}{(m-1)^{\frac{2}{3}}} .
\end{aligned}
$$

Using (2.13) and (2.18)-(2.21) and then (2.14) and (2.15) in (2.16) and (2.17), we deduce that, respectively,

$$
\begin{aligned}
2 b(q) & =\sqrt{z_{1} z_{3}}\left\{\left(\frac{9}{m^{2}}-1\right)^{\frac{1}{3}}-(\alpha \beta)^{\frac{1}{4}} \frac{2 m^{\frac{1}{3}}(3-m)^{\frac{1}{3}}}{(m+3)^{\frac{2}{3}}}\right\} \\
& =\sqrt{z_{1} z_{3}}\left\{\frac{\left(9-m^{2}\right)^{\frac{1}{3}}}{m^{\frac{2}{3}}}-\frac{(m-1)(m+3)}{4 m} \frac{2 m^{\frac{1}{3}}(3-m)^{\frac{1}{3}}}{(m+3)^{\frac{2}{3}}}\right\} \\
& =\sqrt{z_{1} z_{3}} \frac{(3-m)\left(9-m^{2}\right)^{\frac{1}{3}}}{2 m^{\frac{2}{3}}}
\end{aligned}
$$

and

$$
\begin{aligned}
2 c(q) & =\sqrt{z_{1} z_{3}}\left\{\left(m^{2}-1\right)^{\frac{1}{3}}+(\alpha \beta)^{\frac{1}{4}} \frac{2(m+1)^{\frac{1}{3}}}{(m-1)^{\frac{2}{3}}}\right\} \\
& =\sqrt{z_{1} z_{3}}\left\{\left(m^{2}-1\right)^{\frac{1}{3}}+\frac{(m-1)(m+3)(m+1)^{\frac{1}{3}}}{2 m(m-1)^{\frac{2}{3}}}\right\} \\
& =\sqrt{z_{1} z_{3}} \frac{3\left(m^{2}-1\right)^{\frac{1}{3}}(m+1)}{2 m} .
\end{aligned}
$$

Hence, (2.11) and (2.12) have been established.

Theorem 2.2. The cubic theta-function identity (2.5) holds.

Proof. From (2.11) and (2.12),

$$
\begin{aligned}
b^{3}(q)+c^{3}(q) & =\frac{\left(z_{1} z_{3}\right)^{\frac{3}{2}}}{64 m^{3}}\left\{m(3-m)^{3}\left(9-m^{2}\right)+27(m+1)^{3}\left(m^{2}-1\right)\right\} \\
& =\frac{\left(z_{1} z_{3}\right)^{\frac{3}{2}}}{64 m^{3}}\left(m^{2}+6 m-3\right)^{3}=a^{3}(q),
\end{aligned}
$$

by (2.10). This completes the proof. 
Our next task is to state a generalization of Ramanujan's beautiful cubic transformation for ${ }_{2} F_{1}\left(\frac{1}{3}, \frac{2}{3} ; 1 ; x\right)$. E. Goursat [29] derived several cubic transformations for hypergeometric series, but Ramanujan's cubic transformation cannot be deduced from Goursat's results.

Theorem 2.3. For $|x|$ sufficiently small,

$$
\begin{aligned}
{ }_{2} F_{1}(c, & \left., c+\frac{1}{3} ; \frac{3 c+1}{2} ; 1-\left(\frac{1-x}{1+2 x}\right)^{3}\right) \\
& =(1+2 x)^{3 c}{ }_{2} F_{1}\left(c, c+\frac{1}{3} ; \frac{3 c+5}{6} ; x^{3}\right) .
\end{aligned}
$$

Proof. Using MAPLE, we have shown that both sides of (2.22) are solutions of the differential equation

$$
\begin{aligned}
2 x(1 & -x)\left(1+x+x^{2}\right)(1+2 x)^{2} y^{\prime \prime} \\
& -(1+2 x)\left[\left(4 x^{3}-1\right)(3 c+2 x+1)+18 c x\right] y^{\prime}-6 c(3 c+1)(1-x)^{2} y=0
\end{aligned}
$$

This equation has a regular singular point at $x=0$, and the roots of the associated indicial equation are 0 and $(3 c-1) / 2$. Thus, in general, to verify that (2.22) holds, we must show that the values at $x=0$ of the functions and their first derivatives on each side are equal. These values are easily seen to be equal, and so the proof is complete.

Corollary 2.4 (p. 258). For $|x|$ sufficiently small,

$$
{ }_{2} F_{1}\left(\frac{1}{3}, \frac{2}{3} ; 1 ; 1-\left(\frac{1-x}{1+2 x}\right)^{3}\right)=(1+2 x){ }_{2} F_{1}\left(\frac{1}{3}, \frac{2}{3} ; 1 ; x^{3}\right) .
$$

Proof. Set $c=\frac{1}{3}$ in Theorem 2.3.

The Borweins [16] deduced Corollary 2.4 in connection with their cubic analogue of the arithmetic-geometric mean. Neither their proof nor our proof is completely satisfactory, and it would be desirable to have a more natural proof.

Our next goal is to prove a cubic analogue of (1.15). We accomplish this through a series of lemmas.

Lemma 2.5. If $n=3^{m}$, where $m$ is a positive integer, then

$$
{ }_{2} F_{1}\left(\frac{1}{3}, \frac{2}{3} ; 1 ; 1-\frac{b^{3}(q)}{a^{3}(q)}\right)=\frac{a(q)}{a\left(q^{n}\right)}{ }_{2} F_{1}\left(\frac{1}{3}, \frac{2}{3} ; 1 ; 1-\frac{b^{3}\left(q^{n}\right)}{a^{3}\left(q^{n}\right)}\right) .
$$

Proof. Replacing $x$ by $(1-x) /(1+2 x)$ in $(2.23)$, we find that

$$
{ }_{2} F_{1}\left(\frac{1}{3}, \frac{2}{3} ; 1 ; 1-x^{3}\right)=\frac{3}{1+2 x}{ }_{2} F_{1}\left(\frac{1}{3}, \frac{2}{3} ; 1 ;\left(\frac{1-x}{1+2 x}\right)^{3}\right)
$$


Setting $x=b(q) / a(q)$ and employing (2.8) and (2.9), we deduce that

$$
\begin{aligned}
{ }_{2} F_{1}\left(\frac{1}{3}, \frac{2}{3} ; 1 ; 1-\frac{b^{3}(q)}{a^{3}(q)}\right) & =\frac{3 a(q)}{a(q)+2 b(q)}{ }_{2} F_{1}\left(\frac{1}{3}, \frac{2}{3} ; 1 ;\left(\frac{a(q)-b(q)}{a(q)+2 b(q)}\right)^{3}\right) \\
& =\frac{a(q)}{a\left(q^{3}\right)}{ }_{2} F_{1}\left(\frac{1}{3}, \frac{2}{3} ; 1 ; \frac{c^{3}\left(q^{3}\right)}{a^{3}\left(q^{3}\right)}\right) \\
& =\frac{a(q)}{a\left(q^{3}\right)}{ }_{2} F_{1}\left(\frac{1}{3}, \frac{2}{3} ; 1 ; 1-\frac{b^{3}\left(q^{3}\right)}{a^{3}\left(q^{3}\right)}\right),
\end{aligned}
$$

by Theorem 2.2. Iterating this identity $m$ times, we complete the proof of (2.24).

The next result is the Borweins' [16] form of the cubic inversion formula.

Lemma 2.6. We have

$$
{ }_{2} F_{1}\left(\frac{1}{3}, \frac{2}{3} ; 1 ; \frac{c^{3}(q)}{a^{3}(q)}\right)=a(q) .
$$

Proof. Letting $m$ tend to $\infty$ in (2.24), noting that, by (2.2) (or (2.6)) and (2.3) (or 2.8), respectively,

$$
\lim _{n \rightarrow \infty} a\left(q^{n}\right)=1=\lim _{n \rightarrow \infty} b\left(q^{n}\right),
$$

and invoking Theorem 2.2, we deduce (2.26) at once.

Lemma 2.7. If $n=3^{m}$, where $m$ is a positive integer, then

$$
{ }_{2} F_{1}\left(\frac{1}{3}, \frac{2}{3} ; 1 ; \frac{b^{3}(q)}{a^{3}(q)}\right)=\frac{a(q)}{n a\left(q^{n}\right)}{ }_{2} F_{1}\left(\frac{1}{3}, \frac{2}{3} ; 1 ; \frac{b^{3}\left(q^{n}\right)}{a^{3}\left(q^{n}\right)}\right) .
$$

Proof. By Theorem 2.2, (2.25) with $x=c(q) / a(q)$, (2.8), and (2.9),

$$
\begin{aligned}
{ }_{2} F_{1}\left(\frac{1}{3}, \frac{2}{3} ; 1 ; \frac{b^{3}(q)}{a^{3}(q)}\right) & ={ }_{2} F_{1}\left(\frac{1}{3}, \frac{2}{3} ; 1 ; 1-\frac{c^{3}(q)}{a^{3}(q)}\right) \\
& =\frac{3 a(q)}{a(q)+2 c(q)}{ }_{2} F_{1}\left(\frac{1}{3}, \frac{2}{3} ; 1 ;\left(\frac{a(q)-c(q)}{a(q)+2 c(q)}\right)^{3}\right) \\
& =\frac{3 a(q)}{a\left(q^{\frac{1}{3}}\right)}{ }_{2} F_{1}\left(\frac{1}{3}, \frac{2}{3} ; 1 ; \frac{b^{3}\left(q^{\frac{1}{3}}\right)}{a^{3}\left(q^{\frac{1}{3}}\right)}\right) .
\end{aligned}
$$

Replacing $q$ by $q^{3}$ in (2.28), and then iterating the resulting equality a total of $m$ times, we deduce (2.27) to complete the proof.

Lemma 2.8. Let $q_{3}$ be defined by (1.7), and put $F(x)=q_{3}$. Let $n=3^{m}$, where $m$ is a positive integer. Then

$$
F\left(\frac{b^{3}(q)}{a^{3}(q)}\right)=F^{n}\left(\frac{b^{3}\left(q^{n}\right)}{a^{3}\left(q^{n}\right)}\right)
$$

and

$$
F^{n}\left(\frac{c^{3}(q)}{a^{3}(q)}\right)=F\left(\frac{c^{3}\left(q^{n}\right)}{a^{3}\left(q^{n}\right)}\right)
$$


Proof. Dividing (2.24) by (2.27), we deduce that

$$
\frac{{ }_{2} F_{1}\left(\frac{1}{3}, \frac{2}{3} ; 1 ; 1-\frac{b^{3}(q)}{a^{3}(q)}\right)}{{ }_{2} F_{1}\left(\frac{1}{3}, \frac{2}{3} ; 1 ; \frac{b^{3}(q)}{a^{3}(q)}\right)}=n \frac{{ }_{2} F_{1}\left(\frac{1}{3}, \frac{2}{3} ; 1 ; 1-\frac{b^{3}\left(q^{n}\right)}{a^{3}\left(q^{n}\right)}\right)}{{ }_{2} F_{1}\left(\frac{1}{3}, \frac{2}{3} ; 1 ; \frac{b^{3}\left(q^{n}\right)}{a^{3}\left(q^{n}\right)}\right)} .
$$

Multiplying both sides of $(2.31)$ by $-2 \pi / \sqrt{3}$ and then taking the exponential of each side, we obtain (2.29).

Multiply both sides of $(2.31)$ by $-\sqrt{3} /(2 \pi n)$, take the reciprocal of each side, use Theorem 2.2, and then take the exponential of each side. We then arrive at (2.30).

We now establish another fundamental inversion formula.

Lemma 2.9. Let $F$ be defined as in Lemma 2.8. Then

$$
F\left(\frac{c^{3}(q)}{a^{3}(q)}\right)=q .
$$

Proof. Letting $n$ tend to $\infty$ in (2.30) and employing Example 2 in Section 27 of Chapter 11 in Ramanujan's second notebook [4, p. 81], we find that

$$
\begin{aligned}
F\left(\frac{c^{3}(q)}{a^{3}(q)}\right) & =\lim _{n \rightarrow \infty} F^{1 / n}\left(\frac{c^{3}\left(q^{n}\right)}{a^{3}\left(q^{n}\right)}\right) \\
& =\lim _{n \rightarrow \infty}\left(\frac{c^{3}\left(q^{n}\right)}{27 a^{3}\left(q^{n}\right)}\left(1+\frac{5}{9} \frac{c^{3}\left(q^{n}\right)}{a^{3}\left(q^{n}\right)}+\cdots\right)\right)^{1 / n} \\
& =\lim _{n \rightarrow \infty}\left(\left(q^{n}+\cdots\right)\left(1+\frac{5}{9}\left(q^{n}+\cdots\right)+\cdots\right)\right)^{1 / n} \\
& =q,
\end{aligned}
$$

where in the penultimate line we used (2.6) and (2.9).

Theorem 2.10 (p. 258). Let $F$ be defined as in Lemma 2.8. Then

$$
z:={ }_{2} F_{1}\left(\frac{1}{3}, \frac{2}{3} ; 1 ; x\right)=a(F(x))=a\left(q_{3}\right) .
$$

Proof. Let $u=u(x)=b^{3}(F(x)) / a^{3}(F(x))$. Then by Lemma 2.6 and Theorem 2.2,

$$
a(F(x))={ }_{2} F_{1}\left(\frac{1}{3}, \frac{2}{3} ; 1 ; 1-u\right) .
$$

On the other hand, by Lemma 2.9,

$$
F(1-u)=F(x)
$$

or

$$
\frac{{ }_{2} F_{1}\left(\frac{1}{3}, \frac{2}{3} ; 1 ; u\right)}{{ }_{2} F_{1}\left(\frac{1}{3}, \frac{2}{3} ; 1 ; 1-u\right)}=\frac{{ }_{2} F_{1}\left(\frac{1}{3}, \frac{2}{3} ; 1 ; 1-x\right)}{{ }_{2} F_{1}\left(\frac{1}{3}, \frac{2}{3} ; 1 ; x\right)} .
$$

By the monotonicity of ${ }_{2} F_{1}\left(\frac{1}{3}, \frac{2}{3} ; 1 ; x\right)$ on $(0,1)$, it follows that, for $0<x<$ 1 ,

$$
{ }_{2} F_{1}\left(\frac{1}{3}, \frac{2}{3} ; 1 ; 1-u\right)={ }_{2} F_{1}\left(\frac{1}{3}, \frac{2}{3} ; 1 ; x\right) \text {. }
$$


(The argument is given in more complete detail in $[5$, p. 101] with ${ }_{2} F_{1}\left(\frac{1}{3}, \frac{2}{3} ; 1 ; x\right)$ replaced by ${ }_{2} F_{1}\left(\frac{1}{2}, \frac{1}{2} ; 1 ; x\right)$.) In conclusion, (2.32) now follows from (2.33) and (2.35).

Theorem 2.10 is an analogue of the classical theorem, (1.15). Our proof followed along lines similar to those in Ramanujan's development of the classical theory, which is presented in [5, Chapter 17, Sections 3-6].

Corollary 2.11 (p. 258). If $z$ is defined by (2.32) and $q_{3}$ is defined by (1.7), then

$$
z^{2}=1+12 \sum_{n=1}^{\infty} \frac{\chi_{3}(n) n q_{3}^{n}}{1-q_{3}^{n}}
$$

where $\chi_{3}$ denotes the principal character modulo 3 .

Proof. In [5, p. 460, Entry 3(i)], it is shown that

$$
1+12 \sum_{n=1}^{\infty} \frac{n q^{n}}{1-q^{n}}-36 \sum_{n=1}^{\infty} \frac{n q^{3 n}}{1-q^{3 n}}=a^{2}(q) .
$$

Since here $q$ is arbitrary, we may replace $q$ by $q_{3}$ in (2.37). Thus, (2.36) can be deduced from Theorem 2.10 and (2.37).

We conclude this section by offering three additional formulas for $z$.

Theorem 2.12 (p. 257). Let $q=q_{3}$ and $z=z(3)$. Then

$$
z=1+6 \sum_{n=1}^{\infty} \frac{q^{n}}{1+q^{n}+q^{2 n}} .
$$

Proof. By Theorem 2.10 and (2.6),

$$
\begin{aligned}
z & =1+6 \sum_{n=0}^{\infty}\left(\frac{q^{3 n+1}}{1-q^{3 n+1}}-\frac{q^{3 n+2}}{1-q^{3 n+2}}\right) \\
& =1+6 \sum_{n=0}^{\infty} \sum_{m=1}^{\infty}\left(q^{(3 n+1) m}-q^{(3 n+2) m}\right) \\
& =1+6 \sum_{m=1}^{\infty}\left(q^{m}-q^{2 m}\right) \sum_{n=0}^{\infty} q^{3 m n} \\
& =1+6 \sum_{m=1}^{\infty} \frac{q^{m}-q^{2 m}}{1-q^{3 m}} \\
& =1+6 \sum_{m=1}^{\infty} \frac{q^{m}}{1+q^{m}+q^{2 m}},
\end{aligned}
$$

and the proof is complete.

In the middle of page 258, Ramanujan offers two representations for $z$, but one of them involves an unidentified parameter $p$. If $q$ is replaced by $-q$ below, then the parameter $p$ becomes identical to the parameter $p$ in Lemma 5.5 below, as can be seen from (5.11). 
Theorem 2.13 (p. 258). Let $z$ and $q$ be as given above. Put $p=(m-1) / 2$, where $m$ is the multiplier of degree 3 in the classical sense. Then

$$
z=\frac{\varphi^{3}\left(q^{3}\right)}{\varphi(q)}\left(1+4 p+p^{2}\right)=4 \frac{\psi^{3}\left(q^{2}\right)}{\psi\left(q^{6}\right)}-3 \frac{\varphi^{3}\left(q^{3}\right)}{\varphi(q)} .
$$

Proof. Our proofs will be effected in the classical base $q$. We first assume that the second equality holds and then solve it for $p$. Let $\beta$ have degree 3. By $(2.13)$,

$$
\begin{aligned}
1+4 p+p^{2} & =4 \frac{\psi^{3}\left(q^{2}\right)}{\psi\left(q^{6}\right)} \frac{\varphi(q)}{\varphi^{3}\left(q^{3}\right)}-3 \\
& =m^{2} \frac{(\alpha / q)^{\frac{3}{4}}}{\left(\beta / q^{3}\right)^{\frac{1}{4}}}-3=m^{2}\left(\frac{3+m}{2 m}\right)^{2}-3,
\end{aligned}
$$

by (2.14) and (2.15). Solving (2.39) for $p$, we easily find that $p=(m-1) / 2$, as claimed.

Secondly, we prove the first equality in (2.38). By the same reasoning as used in (2.39),

$$
\begin{aligned}
4 \frac{\psi^{3}\left(q^{2}\right)}{\psi\left(q^{6}\right)}-3 \frac{\varphi^{3}\left(q^{3}\right)}{\varphi(q)} & =\frac{z_{1}^{\frac{3}{2}}}{z_{3}^{\frac{1}{2}}} \frac{\alpha^{\frac{3}{4}}}{\beta^{\frac{1}{4}}}-3 \frac{z_{3}^{\frac{3}{2}}}{z_{1}^{\frac{1}{2}}} \\
& =\sqrt{z_{1} z_{3}}\left(m\left(\frac{3+m}{2 m}\right)^{2}-\frac{3}{m}\right) \\
& =\sqrt{z_{1} z_{3}}\left(\frac{m^{2}+6 m-3}{4 m}\right) \\
& =a(q),
\end{aligned}
$$

by (2.10). Appealing to Theorem 2.10, we complete the proof.

\section{THE PRINCIPLES OF TRIPLICATION AND TRIMIDIATION}

In Sections 3 and 4 , for brevity, we set $q=q_{3}$, and $z=z(3 ; x)$ (unless otherwise stated).

In the classical theory of elliptic functions, the processes of duplication and dimidiation, which rest upon Landen's transformation, are very useful in obtaining formulas from previously derived formulas when $q$ is replaced by $q^{2}$ or $\sqrt{q}$, respectively. These procedures are described in detail in [5, Chapter 17, Section 13], where many applications are given. We now show that Ramanujan's cubic transformation, Corollary 2.4 , can be employed to devise the new processes of triplication and trimidiation.

Theorem 3.1. Let $x, q_{3}=q=q(x)$, and $z(3 ; x)=z$ be as given in (1.7) and (1.6), respectively. Set $x=t^{3}$. Suppose that a relation of the form

$$
\Omega\left(t^{3}, q, z\right)=0
$$

holds. Then we have the triplication formula

$$
\Omega\left(\left\{\frac{1-\left(1-t^{3}\right)^{\frac{1}{3}}}{1+2\left(1-t^{3}\right)^{\frac{1}{3}}}\right\}^{3}, q^{3}, \frac{1}{3}\left\{1+2\left(1-t^{3}\right)^{\frac{1}{3}}\right\} z\right)=0
$$


and the trimidiation formula

$$
\Omega\left(1-\left(\frac{1-t}{1+2 t}\right)^{3}, q^{\frac{1}{3}},(1+2 t) z\right)=0 .
$$

Proof. Set

$$
t^{\prime 3}=1-\left(\frac{1-t}{1+2 t}\right)^{3}
$$

Therefore,

$$
t=\frac{1-\left(1-t^{\prime 3}\right)^{\frac{1}{3}}}{1+2\left(1-t^{\prime 3}\right)^{\frac{1}{3}}}
$$

By Corollary 2.4,

$$
\begin{aligned}
z^{\prime}:=z\left(t^{\prime 3}\right) & ={ }_{2} F_{1}\left(\frac{1}{3}, \frac{2}{3} ; 1 ; t^{\prime 3}\right) \\
& ={ }_{2} F_{1}\left(\frac{1}{3}, \frac{2}{3} ; 1 ; 1-\left(\frac{1-t}{1+2 t}\right)^{3}\right) \\
& =(1+2 t){ }_{2} F_{1}\left(\frac{1}{3}, \frac{2}{3} ; 1 ; t^{3}\right) \\
& =(1+2 t) z\left(t^{3}\right) .
\end{aligned}
$$

Also, by (1.7), (3.4), (2.25), and (2.23),

$$
\begin{aligned}
q^{\prime}:=q\left(t^{\prime 3}\right) & =\exp \left(-\frac{2 \pi}{\sqrt{3}} \frac{{ }_{2} F_{1}\left(\frac{1}{3}, \frac{2}{3} ; 1 ; 1-t^{\prime 3}\right)}{{ }_{2} F_{1}\left(\frac{1}{3}, \frac{2}{3} ; 1 ; t^{\prime 3}\right)}\right) \\
& =\exp \left(-\frac{2 \pi}{\sqrt{3}} \frac{{ }_{2} F_{1}\left(\frac{1}{3}, \frac{2}{3} ; 1 ;\left(\frac{1-t}{1+2 t}\right)^{3}\right)}{{ }_{1}\left(\frac{1}{3}, \frac{2}{3} ; 1 ; 1-\left(\frac{1-t}{1+2 t}\right)^{3}\right)}\right) \\
& =\exp \left(-\frac{2 \pi}{3 \sqrt{3}} \frac{{ }_{2} F_{1}\left(\frac{1}{3}, \frac{2}{3} ; 1 ; 1-t^{3}\right)}{{ }_{2} F_{1}\left(\frac{1}{3}, \frac{2}{3} ; 1 ; t^{3}\right)}\right) \\
& =q^{\frac{1}{3}}\left(t^{3}\right) .
\end{aligned}
$$

Thus, suppose that (3.1) holds. Then by (3.5)-(3.7), we obtain (3.2), but with $t, q$, and $z$ replaced by $t^{\prime}, q^{\prime}$, and $z^{\prime}$, respectively.

On the other hand, suppose that (3.1) holds with $t, q$, and $z$ replaced by $t^{\prime}, q^{\prime}$, and $z^{\prime}$, respectively. Then by (3.4), (3.6), and (3.7), it follows that (3.3) holds.

Corollary 3.2. With $q$ and $z$ as above,

$$
b(q)=(1-x)^{\frac{1}{3}} z
$$

and

$$
c(q)=x^{\frac{1}{3}} z
$$


Proof. By (2.8), Theorem 2.10, and the process of triplication,

$$
b(q)=\frac{1}{2}\left(3 \cdot \frac{1}{3}\left\{1+2(1-x)^{\frac{1}{3}}\right\} z-z\right)=(1-x)^{\frac{1}{3}} z,
$$

while by (2.9), Theorem 2.10 , and the process of trimidiation,

$$
c(q)=\frac{1}{2}\left(\left(1+2 x^{\frac{1}{3}}\right) z-z\right)=x^{\frac{1}{3}} z .
$$

Theorem 3.3. Recall that $f(-q)$ is defined by (1.14). Then for any base $q$,

$$
q f^{24}(-q)=\frac{1}{27} b^{9}(q) c^{3}(q) \text {. }
$$

Proof. All calculations below pertain to the classical base $q$. 124],

By Entry 12(ii) in Chapter 17 of Ramanujan's second notebook [35], [5, p.

$$
q f^{24}(-q)=\frac{1}{16} z_{1}^{12} \alpha(1-\alpha)^{4} .
$$

It thus suffices to show that the right side of (3.8) is equal to the right side of (3.9). To do this, we use the parameterizations for $b(q)$ and $c(q)$ given by (2.11) and (2.12), respectively. It will then be necessary to express the functions of $m$ arising in (2.11) and (2.12) in terms of $\alpha$ and $\beta$. In addition to (2.14) and $(2.15)$, we need the parameterizations

$$
1-\alpha=\frac{(m+1)(3-m)^{3}}{16 m^{3}}
$$

and

$$
1-\beta=\frac{(m+1)^{3}(3-m)}{16 m},
$$

given in (5.5) of Chapter 19 of [5, p. 233]. Direct calculations yield

$$
\begin{aligned}
& 9-m^{2}=4 m^{2} \frac{\alpha^{\frac{3}{8}}(1-\alpha)^{\frac{3}{8}}}{\beta^{\frac{1}{8}}(1-\beta)^{\frac{1}{8}}}, \\
& 3-m=2 m \frac{(1-\alpha)^{\frac{3}{8}}}{(1-\beta)^{\frac{1}{8}}}, \\
& m+1=2 \frac{(1-\beta)^{\frac{3}{8}}}{(1-\alpha)^{\frac{1}{8}}}
\end{aligned}
$$

and

$$
m^{2}-1=4 \frac{\beta^{\frac{3}{8}}(1-\beta)^{\frac{3}{8}}}{\alpha^{\frac{1}{8}}(1-\alpha)^{\frac{1}{8}}} .
$$

Hence, by (2.11), (3.12), and (3.13),

$$
\begin{aligned}
b(q) & =\frac{z_{1}}{4 m^{\frac{7}{6}}} 2 m \frac{(1-\alpha)^{\frac{3}{8}}}{(1-\beta)^{\frac{1}{8}}}\left(4 m^{2}\right)^{\frac{1}{3}} \frac{\alpha^{\frac{1}{8}}(1-\alpha)^{\frac{1}{8}}}{\beta^{\frac{1}{24}}(1-\beta)^{\frac{1}{24}}} \\
& =\frac{z_{1} m^{\frac{1}{2}} \alpha^{\frac{1}{8}}(1-\alpha)^{\frac{1}{2}}}{2^{\frac{1}{3}} \beta^{\frac{1}{24}}(1-\beta)^{\frac{1}{6}}} .
\end{aligned}
$$


By (2.12), (3.14), and (3.15),

$$
\begin{aligned}
c(q) & =\frac{3 z_{1}}{4 m^{\frac{3}{2}}} \frac{2(1-\beta)^{\frac{3}{8}}}{(1-\alpha)^{\frac{1}{8}}} \frac{4^{\frac{1}{3}} \beta^{\frac{1}{8}}(1-\beta)^{\frac{1}{8}}}{\alpha^{\frac{1}{24}}(1-\alpha)^{\frac{1}{24}}} \\
& =\frac{3 z_{1} \beta^{\frac{1}{8}}(1-\beta)^{\frac{1}{2}}}{2^{\frac{1}{3}} m^{\frac{3}{2}} \alpha^{\frac{1}{24}}(1-\alpha)^{\frac{1}{6}}} .
\end{aligned}
$$

It now follows easily from (3.16) and (3.17) that

$$
\frac{1}{27} b^{9}(q) c^{3}(q)=\frac{1}{16} z_{1}^{12} \alpha(1-\alpha)^{4},
$$

which, by (3.9), completes the proof.

Corollary 3.4 (p. 257). Let $q=q_{3}$, and let $z$ be as in Theorem 2.10. Then

$$
q^{\frac{1}{24}} f(-q)=\sqrt{z} 3^{-\frac{1}{8}} x^{\frac{1}{24}}(1-x)^{\frac{1}{8}} .
$$

Proof. By Theorem 3.3 and Corollary 3.2,

$$
q f^{24}(-q)=\frac{1}{27}(1-x)^{3} x z^{12}
$$

from which (3.18) follows.

Corollary 3.5 (p. 257). With the same notation as in Corollary 3.4,

$$
q^{\frac{1}{8}} f\left(-q^{3}\right)=\sqrt{z} 3^{-\frac{3}{8}} x^{\frac{1}{8}}(1-x)^{\frac{1}{24}} .
$$

Proof. Applying to (3.18) the process of triplication enunciated in Theorem 3.1, we deduce that

$$
\begin{aligned}
q^{\frac{1}{8}} f\left(-q^{3}\right)= & \sqrt{z} \frac{\left\{1+2(1-x)^{\frac{1}{3}}\right\}^{\frac{1}{2}}}{3^{\frac{5}{8}}}\left\{\frac{1-(1-x)^{\frac{1}{3}}}{1+2(1-x)^{\frac{1}{3}}}\right\}^{\frac{1}{8}} \\
& \cdot\left\{\frac{\left(1+2(1-x)^{\frac{1}{3}}\right)^{3}-\left(1-(1-x)^{\frac{1}{3}}\right)^{3}}{\left(1+2(1-x)^{\frac{1}{3}}\right)^{3}}\right. \\
= & \sqrt{z} 3^{-\frac{3}{8}}\left\{1-(1-x)^{\frac{1}{3}}\right\}^{\frac{1}{8}}(1-x)^{\frac{1}{24}}\left\{1+(1-x)^{\frac{1}{3}}+(1-x)^{\frac{2}{3}}\right\}^{\frac{1}{8}} \\
= & \sqrt{z} 3^{-\frac{3}{8}}\{1-(1-x)\}^{\frac{1}{8}}(1-x)^{\frac{1}{24}} \\
= & \sqrt{z} 3^{-\frac{3}{8}}(1-x)^{\frac{1}{24}} x^{\frac{1}{8}},
\end{aligned}
$$

as desired.

4. The Eisenstein SERIES $L, M$, AND $N$

We now recall Ramanujan's definitions of $L, M$, and $N$, first defined in Chapter 15 of his second notebook [35], [4, p. 318] and thoroughly studied by him, especially in his paper [34], [36, pp. 136-162], where the notations $P, Q$, 
and $R$ are used instead of $L, M$, and $N$, respectively. Thus,

$$
\begin{aligned}
L(q) & :=1-24 \sum_{n=1}^{\infty} \frac{n q^{n}}{1-q^{n}}, \\
M(q) & :=1+240 \sum_{n=1}^{\infty} \frac{n^{3} q^{n}}{1-q^{n}},
\end{aligned}
$$

and

$$
N(q):=1-504 \sum_{n=1}^{\infty} \frac{n^{5} q^{n}}{1-q^{n}} .
$$

We first derive an analogue of Entry 9(iv) in Chapter 17 in Ramanujan's second notebook [35], [5, p. 120].

Lemma 4.1. Let $q=q_{3}$ be defined by (1.7), and let $z$ be as in Theorem 2.10. Then

$$
L(q)=(1-4 x) z^{2}+12 x(1-x) z \frac{d z}{d x} .
$$

Proof. By logarithmic differentiation,

$$
\begin{aligned}
q \frac{d}{d q} \log \left(q f^{24}(-q)\right) & =q \frac{d}{d q} \log \left(q \prod_{n=1}^{\infty}\left(1-q^{n}\right)^{24}\right) \\
& =1-24 \sum_{n=1}^{\infty} \frac{n q^{n}}{1-q^{n}} \\
& =L(q) .
\end{aligned}
$$

On the other hand, by Corollary 3.4 ,

$$
\begin{aligned}
q \frac{d}{d q} \log \left(q f^{24}(-q)\right) & =q \frac{d}{d q} \log \left(\frac{1}{27} z^{12} x(1-x)^{3}\right) \\
& =q \frac{d}{d x} \log \left(\frac{1}{27} z^{12} x(1-x)^{3}\right) \frac{d x}{d q}
\end{aligned}
$$

Now by Entry 30 in Chapter 11 of Ramanujan's second notebook [35], [4, p. 87],

$$
\frac{d}{d x}\left(\frac{2 \pi}{\sqrt{3}} \frac{{ }_{2} F_{1}\left(\frac{1}{3}, \frac{2}{3} ; 1 ; 1-x\right)}{{ }_{2} F_{1}\left(\frac{1}{3}, \frac{2}{3} ; 1 ; x\right)}\right)=-\frac{1}{x(1-x) z^{2}} .
$$

Thus,

$$
\frac{d q}{d x}=\frac{q}{x(1-x) z^{2}}
$$

Using (4.4) in (4.3), we deduce that

$$
\begin{aligned}
q \frac{d}{d q} \log \left(q f^{24}(-q)\right) & =x(1-x) z^{2}\left(\frac{12}{z} \frac{d z}{d x}+\frac{1}{x}-\frac{3}{1-x}\right) \\
& =12 x(1-x) z \frac{d z}{d x}+(1-4 x) z^{2}
\end{aligned}
$$

Combining (4.2) and (4.5), we arrive at (4.1) to complete the proof. 
Theorem 4.2 (p. 257). We have

$$
M(q)=z^{4}(1+8 x) .
$$

Proof. From [34], [36, p. 330] or from [4, p. 330, Entry 13],

$$
q \frac{d L}{d q}=\frac{1}{12}\left\{L^{2}(q)-M(q)\right\} .
$$

Thus, by (4.4) and Lemma 4.1,

$$
\begin{aligned}
M(q)= & L^{2}(q)-12 x(1-x) z^{2} \frac{d L}{d x} \\
= & (1-4 x)^{2} z^{4}+24 x(1-x)(1-4 x) z^{3} \frac{d z}{d x}+144 x^{2}(1-x)^{2} z^{2}\left(\frac{d z}{d x}\right)^{2} \\
& -12 x(1-x) z^{2}\left(-4 z^{2}+2(1-4 x) z \frac{d z}{d x}\right. \\
& \left.+12 x(1-x)\left(\frac{d z}{d x}\right)^{2}+12 z \frac{2}{9} z\right),
\end{aligned}
$$

where we have employed the differential equation for $z[2, \mathrm{p} .1]$

$$
\frac{d}{d x}\left\{x(1-x) \frac{d z}{d x}\right\}=\frac{2}{9} z
$$

Upon simplifying the equality above, we deduce (4.6).

Theorem 4.3 (p. 257). We have

$$
N(q)=z^{6}\left(1-20 x-8 x^{2}\right) .
$$

Proof. From [34], [36, p. 142] or from [4, p. 330, Entry 13],

$$
q \frac{d M}{d q}=\frac{1}{3}\{L(q) M(q)-N(q)\} .
$$

Thus, by (4.4), Lemma 4.1, and Theorem 4.2,

$$
\begin{aligned}
N(q)= & L(q) M(q)-3 x(1-x) z^{2} \frac{d M}{d x} \\
= & \left\{(1-4 x) z^{2}+12 x(1-x) z \frac{d z}{d x}\right\} z^{4}(1+8 x) \\
& -3 x(1-x) z^{2}\left\{4 z^{3}(1+8 x) \frac{d z}{d x}+8 z^{4}\right\} \\
= & z^{6}\{(1-4 x)(1+8 x)-24 x(1-x)\} \\
= & z^{6}\left(1-20 x-8 x^{2}\right),
\end{aligned}
$$

and so (4.8) has been proved.

Theorem 4.4 (p. 257). We have

$$
M\left(q^{3}\right)=z^{4}\left(1-\frac{8}{9} x\right) .
$$


Proof. Apply the process of triplication to (4.6). Thus, by Theorem 3.1,

$$
\begin{aligned}
M\left(q^{3}\right) & =\frac{1}{81} z^{4}\left(1+2(1-x)^{\frac{1}{3}}\right)^{4}\left(1+8\left\{\frac{1-(1-x)^{\frac{1}{3}}}{1+2(1-x)^{\frac{1}{3}}}\right\}^{3}\right) \\
& =\frac{1}{9} z^{4}\left(1+2(1-x)^{\frac{1}{3}}\right)\left(1-2(1-x)^{\frac{1}{3}}+4(1-x)^{\frac{2}{3}}\right) \\
& =\frac{1}{9} z^{4}(1+8(1-x)) \\
& =\frac{1}{9} z^{4}(9-8 x),
\end{aligned}
$$

and the proof is complete.

Theorem 4.5 (p. 257). We have

$$
N\left(q^{3}\right)=z^{6}\left(1-\frac{4}{3} x+\frac{8}{27} x^{2}\right) .
$$

Proof. Applying the process of triplication to (4.8), we find that

$$
\begin{aligned}
N\left(q^{3}\right) & =\frac{z^{6}}{3^{6}}\left(1+2(1-x)^{\frac{1}{3}}\right)^{6}\left(1-20\left\{\frac{1-(1-x)^{\frac{1}{3}}}{1+2(1-x)^{\frac{1}{3}}}\right\}^{3}-8\left\{\frac{1-(1-x)^{\frac{1}{3}}}{1+2(1-x)^{\frac{1}{3}}}\right\}^{6}\right) \\
& =\frac{z^{6}}{3^{6}}\left\{\left(1+2(1-x)^{\frac{1}{3}}\right)^{6}-20\left(1+2(1-x)^{\frac{1}{3}}\right)^{3}\left(1-(1-x)^{\frac{1}{3}}\right)^{3}\right. \\
& =\frac{z^{6}}{3^{6}}\left\{-27+540(1-x)+216(1-x)^{2}\right\} \\
& =\frac{z^{6}}{3^{6}}\left(729-972 x+216 x^{2}\right) \\
& =z^{6}\left(1-\frac{4}{3} x+\frac{8}{27} x^{2}\right) .
\end{aligned}
$$

We complete this section by offering a remarkable formula for $z^{4}$ and an identity involving the 6th powers of the Borweins' cubic theta-functions $b(q)$ and $c(q)$.

Corollary 4.6. We have

$$
10 z^{4}=9 M\left(q^{3}\right)+M(q) .
$$

Proof. Using Theorems 4.2 and 4.4 on the right side of (4.9), we easily establish the desired result.

Corollary 4.7. We have

$$
28\left\{b^{6}(q)-c^{6}(q)\right\}=27 N\left(q^{3}\right)+N(q) .
$$

Proof. Using Theorems 4.3 and 4.5 on the right side of (4.10), and also employing Corollary 3.2 , we readily deduce $(4.10)$. 


\section{A HYPERGEOMETRIC TRANSFORMATION AND ASSOCIATED TRANSFER PRINCIPLE}

We shall prove a new transformation formula relating the hypergeometric functions $z(2)$ and $z(3)$ and employ it to establish a means for transforming formulas in the theory of signature 2 to that in signature 3 , and conversely. We first need to establish several formulas relating the functions $\varphi(q), \psi(q)$, and $f(-q)$ with $a(q), b(q)$, and $c(q)$.

Let, as customary,

$$
(a ; q)_{\infty}:=(1-a)(1-a q)\left(1-a q^{2}\right) \cdots, \quad|q|<1 .
$$

For any integer $n$, also set

$$
(a ; q)_{n}=\frac{(a ; q)_{\infty}}{\left(a ; q^{n}\right)_{\infty}}
$$

From the Jacobi triple product identity [5, pp. 36,37],

$$
\begin{aligned}
\varphi(-q) & =(q ; q)_{\infty}\left(q ; q^{2}\right)_{\infty}, \\
\psi(q) & =\frac{\left(q^{2} ; q^{2}\right)_{\infty}}{\left(q ; q^{2}\right)_{\infty}},
\end{aligned}
$$

and

$$
f(-q)=(q ; q)_{\infty}
$$

Lemma 5.1. We have

$$
\begin{aligned}
b(q) & =\frac{f^{3}(-q)}{f\left(-q^{3}\right)}, \\
c(q) & =3 q^{\frac{1}{3}} \frac{f^{3}\left(-q^{3}\right)}{f(-q)}, \\
3 \frac{c\left(q^{2}\right)}{c^{2}(q)} & =\frac{\varphi(-q)}{\varphi^{3}\left(-q^{3}\right)}, \\
\frac{c^{2}\left(q^{4}\right)}{3 c\left(q^{2}\right)} & =q^{2} \frac{\psi^{3}\left(q^{6}\right)}{\psi\left(q^{2}\right)},
\end{aligned}
$$

and

$$
\frac{c^{2}\left(q^{4}\right)}{c^{2}(q)}=q^{2} \frac{\psi^{3}\left(q^{6}\right) \varphi(-q)}{\psi\left(q^{2}\right) \varphi^{3}\left(-q^{3}\right)} .
$$

Proof. First, (5.4) and (5.5) follow directly from Corollaries 3.2, 3.4, and 3.5.

Next, from (5.5) and (5.3),

$$
3 \frac{c\left(q^{2}\right)}{c^{2}(q)}=\frac{\left(q^{6} ; q^{6}\right)_{\infty}^{3}(q ; q)_{\infty}^{2}}{\left(q^{2} ; q^{2}\right)_{\infty}\left(q^{3} ; q^{3}\right)_{\infty}^{6}}
$$

Using (5.1), we readily find that the right side above equals $\varphi(-q) / \varphi^{3}\left(-q^{3}\right)$, and the proof of $(5.6)$ is complete.

Again, from (5.5) and (5.3),

$$
\frac{c^{2}\left(q^{4}\right)}{3 c\left(q^{2}\right)}=q^{2} \frac{\left(q^{12} ; q^{12}\right)_{\infty}^{6}\left(q^{2} ; q^{2}\right)_{\infty}}{\left(q^{4} ; q^{4}\right)_{\infty}^{2}\left(q^{6} ; q^{6}\right)_{\infty}^{3}}
$$

which, by (5.2), is seen to equal $q^{2} \psi^{3}\left(q^{6}\right) / \psi\left(q^{2}\right)$. Thus, (5.7) is proved. 
Lastly, (5.8) follows from combining (5.6) and (5.7).

Lemma 5.2. We have

$$
1-\frac{\varphi^{2}(-q)}{\varphi^{2}\left(-q^{3}\right)}=4 \frac{c\left(q^{4}\right)}{c(q)}
$$

Proof. By (5.8), we want to prove that

$$
\varphi^{2}\left(-q^{3}\right)-\varphi^{2}(-q)=4 \varphi^{2}\left(-q^{3}\right)\left(q^{2} \frac{\psi^{3}\left(q^{6}\right) \varphi(-q)}{\psi\left(q^{2}\right) \varphi^{3}\left(-q^{3}\right)}\right)^{\frac{1}{2}} .
$$

By Entry 10(ii) of Chapter 17 of the second notebook [5, p. 122],

$$
\varphi(-q)=\sqrt{z_{1}}(1-\alpha)^{\frac{1}{4}} \quad \text { and } \quad \varphi\left(-q^{3}\right)=\sqrt{z_{3}}(1-\beta)^{\frac{1}{4}},
$$

where $\beta$ has degree 3 over $\alpha$. Thus, by (2.13) and (5.9), it suffices to prove that

$$
z_{3}(1-\beta)^{\frac{1}{2}}-z_{1}(1-\alpha)^{\frac{1}{2}}=2 z_{3}(1-\beta)^{\frac{1}{2}}\left(\frac{\beta^{3}}{\alpha}\right)^{\frac{1}{8}}\left(\frac{1-\alpha}{(1-\beta)^{3}}\right)^{\frac{1}{8}}
$$

Since $m=z_{1} / z_{3}$, the last equality is equivalent to the equality,

$$
1-m\left(\frac{1-\alpha}{1-\beta}\right)^{\frac{1}{2}}=2\left(\frac{\beta^{3}}{\alpha}\right)^{\frac{1}{8}}\left(\frac{1-\alpha}{(1-\beta)^{3}}\right)^{\frac{1}{8}} \text {. }
$$

By (2.14), (2.15), (3.10), and (3.11), the last equality can be written entirely in terms of $m$, namely,

$$
1-m \frac{3-m}{m(m+1)}=2 \frac{m-1}{2} \frac{2}{m+1} .
$$

This equality is trivially verified, and so the proof is complete.

Lemma 5.3. We have

$$
1+\frac{\psi^{2}\left(q^{2}\right)}{q \psi^{2}\left(q^{6}\right)}=\frac{c(q)}{c\left(q^{4}\right)} .
$$

Proof. By (5.8), the proposed identity is equivalent to the identity

$$
q \psi^{2}\left(q^{6}\right)+\psi^{2}\left(q^{2}\right)=\left(\frac{\psi\left(q^{2}\right) \psi\left(q^{6}\right) \varphi^{3}\left(-q^{3}\right)}{\varphi(-q)}\right)^{\frac{1}{2}} .
$$

By (2.13) and (5.9), the previous identity is equivalent to the identity,

$$
1+m\left(\frac{\alpha}{\beta}\right)^{\frac{1}{2}}=2\left(\frac{\alpha}{\beta^{3}}\right)^{\frac{1}{8}}\left(\frac{(1-\beta)^{3}}{1-\alpha}\right)^{\frac{1}{8}} .
$$

By (2.14), (2.15), (3.10), and (3.11), the last equality can be expressed completely in terms of $m$ as

$$
1+m \frac{3+m}{m(m-1)}=2 \frac{2}{m-1} \frac{m+1}{2} .
$$

Since this last equality is trivially verified, the proof is complete.

Lemma 5.4. We have

$$
a(q)=\frac{\varphi^{3}\left(-q^{3}\right)}{\varphi(-q)}+4 q \frac{\psi^{3}\left(q^{3}\right)}{\psi(q)}
$$


Proof. By Entry 11(i) in Chapter 17 [5, p. 123],

$$
\psi(q)=\sqrt{\frac{1}{2} z_{1}}(\alpha / q)^{\frac{1}{8}} \quad \text { and } \quad \psi\left(q^{3}\right)=\sqrt{\frac{1}{2} z_{3}}\left(\beta / q^{3}\right)^{\frac{1}{8}}
$$

Thus, by Entry 3(i) in Chapter 21 of Ramanujan's second notebook [5, p. 460], (5.10), (2.14), (2.15), (3.10), and (3.11),

$$
\begin{aligned}
a(q) & =\frac{\psi^{3}(q)}{\psi\left(q^{3}\right)}+3 q \frac{\psi^{3}\left(q^{3}\right)}{\psi(q)} \\
& =\frac{z_{1}^{\frac{3}{2}}}{2 z_{3}^{\frac{1}{2}}}\left(\frac{\alpha^{3}}{\beta}\right)^{\frac{1}{8}}+3 q \frac{\psi^{3}\left(q^{3}\right)}{\psi(q)} \\
& =\frac{z_{3}^{\frac{3}{2}}}{z_{1}^{\frac{1}{2}}} \frac{m^{2}+3 m}{4}+3 q \frac{\psi^{3}\left(q^{3}\right)}{\psi(q)} \\
& =\frac{z_{3}^{\frac{3}{2}}}{z_{1}^{\frac{1}{2}}}\left(\frac{(m+1)^{2}}{4}+\frac{m-1}{4}\right)+3 q \frac{\psi^{3}\left(q^{3}\right)}{\psi(q)} \\
& =\frac{z_{3}^{\frac{3}{2}}}{z_{1}^{\frac{1}{2}}}\left(\left(\frac{(1-\beta)^{3}}{1-\alpha}\right)^{\frac{1}{4}}+\frac{1}{2}\left(\frac{\beta^{3}}{\alpha}\right)^{\frac{1}{8}}\right)+3 q \frac{\psi^{3}\left(q^{3}\right)}{\psi(q)} .
\end{aligned}
$$

By (5.9) and (5.10), the right-hand side above equals

$$
\frac{\varphi^{3}\left(-q^{3}\right)}{\varphi(-q)}+q \frac{\psi^{3}\left(q^{3}\right)}{\psi(q)}+3 q \frac{\psi^{3}\left(q^{3}\right)}{\psi(q)} .
$$

The desired result now follows.

Lemma 5.5. If $p:=p(q):=-2 c\left(q^{4}\right) / c(q)$, then

$$
\begin{aligned}
1+2 p & =\frac{\varphi^{2}(-q)}{\varphi^{2}\left(-q^{3}\right)} \\
2+p & =2 \frac{c\left(q^{4}\right)}{c(q)} \frac{\psi^{2}\left(q^{2}\right)}{q \psi^{2}\left(q^{6}\right)} \\
1+p & =-\frac{c(-q)}{c(q)}=\frac{c^{3}\left(q^{2}\right)}{c^{2}(q) c\left(q^{4}\right)}
\end{aligned}
$$

and

$$
1+p+p^{2}=3 \frac{a\left(q^{2}\right) c\left(q^{2}\right)}{c^{2}(q)} .
$$

Proof. Equations (5.11) and (5.12) follow from Lemmas 5.2 and 5.3, respectively.

As observed in [18], it follows from the definition (2.4) of $c(q)$ that

$$
c(q)+c(-q)=2 c\left(q^{4}\right) .
$$


4184

B. C. BERNDT, S. BHARGAVA, AND F. G. GARVAN

Thus, by (5.15), (5.5), and (5.3),

$$
\begin{aligned}
1+p=-\frac{c(-q)}{c(q)} & =\frac{\left(-q^{3} ;-q^{3}\right)_{\infty}^{3}(q ; q)_{\infty}}{(-q ;-q)_{\infty}\left(q^{3} ; q^{3}\right)_{\infty}^{3}} \\
& =\frac{\left(-q^{3} ; q^{6}\right)_{\infty}^{3}\left(q^{6} ; q^{6}\right)_{\infty}^{3}(q ; q)_{\infty}}{\left(-q ; q^{2}\right)_{\infty}\left(q^{2} ; q^{2}\right)_{\infty}\left(q^{3} ; q^{3}\right)_{\infty}^{3}} \\
& =\frac{\left(q^{6} ; q^{12}\right)_{\infty}^{3}\left(q^{6} ; q^{6}\right)_{\infty}^{3}(q ; q)_{\infty}\left(q ; q^{2}\right)_{\infty}}{\left(q^{3} ; q^{6}\right)_{\infty}^{3}\left(q^{2} ; q^{4}\right)_{\infty}\left(q^{2} ; q^{2}\right)_{\infty}\left(q^{3} ; q^{3}\right)_{\infty}^{3}} \\
& =\frac{\left(q^{6} ; q^{6}\right)_{\infty}^{9}(q ; q)_{\infty}^{2}\left(q^{4} ; q^{4}\right)_{\infty}}{\left(q^{2} ; q^{2}\right)_{\infty}^{3}\left(q^{3} ; q^{3}\right)_{\infty}^{6}\left(q^{12} ; q^{12}\right)_{\infty}^{3}} \\
& =\frac{c^{3}\left(q^{2}\right)}{c^{2}(q) c\left(q^{4}\right)}
\end{aligned}
$$

Thus, (5.13) has been verified.

Lastly, by Lemma 5.4, (5.6), and (5.7),

$$
a\left(q^{2}\right)=\frac{1}{3}\left(\frac{c^{2}\left(q^{2}\right)}{c\left(q^{4}\right)}+4 \frac{c^{2}\left(q^{4}\right)}{c\left(q^{2}\right)}\right) .
$$

Hence, by (5.13) and (5.16),

$$
\begin{aligned}
1+p+p^{2} & =\frac{c^{3}\left(q^{2}\right)}{c^{2}(q) c\left(q^{4}\right)}+4 \frac{c^{2}\left(q^{4}\right)}{c^{2}(q)} \\
& =\frac{c\left(q^{2}\right)}{c^{2}(q)}\left(\frac{c^{2}\left(q^{2}\right)}{c\left(q^{4}\right)}+4 \frac{c^{2}\left(q^{4}\right)}{c\left(q^{2}\right)}\right) \\
& =\frac{3 c\left(q^{2}\right) a\left(q^{2}\right)}{c^{2}(q)}
\end{aligned}
$$

which proves (5.14).

Theorem 5.6 (p. 258). If

$$
\alpha=\frac{p^{3}(2+p)}{1+2 p} \quad \text { and } \quad \beta=\frac{27 p^{2}(1+p)^{2}}{4\left(1+p+p^{2}\right)^{3}},
$$

then, for $0 \leq p<1$,

$$
\left(1+p+p^{2}\right)_{2} F_{1}\left(\frac{1}{2}, \frac{1}{2} ; 1 ; \alpha\right)=\sqrt{1+2 p}_{2} F_{1}\left(\frac{1}{3}, \frac{2}{3} ; 1 ; \beta\right) .
$$

Proof. By Lemma 5.5 and (5.8),

$$
\begin{aligned}
\alpha & =-16 \frac{c^{4}\left(q^{4}\right)}{c^{4}(q)} \frac{\varphi^{2}\left(-q^{3}\right)}{\varphi^{2}(-q)} \frac{\psi^{2}\left(q^{2}\right)}{q \psi^{2}\left(q^{6}\right)} \\
& =-16 q^{3} \frac{\psi^{4}\left(q^{6}\right)}{\varphi^{4}\left(-q^{3}\right)}=1-\frac{\varphi^{4}\left(q^{3}\right)}{\varphi^{4}\left(-q^{3}\right)}
\end{aligned}
$$

by Jacobi's identity for fourth powers, (2.1), with $q$ replaced by $q^{3}$. Thus [5, p. 98, Entry 3], with $q$ replaced by $-q^{3}$,

$$
{ }_{2} F_{1}\left(\frac{1}{2}, \frac{1}{2} ; 1 ; \alpha\right)=\varphi^{2}\left(-q^{3}\right) \text {. }
$$


Also, by Lemma 5.5,

$$
\beta=\frac{c^{3}\left(q^{2}\right)}{a^{3}\left(q^{2}\right)}
$$

Thus, by Lemma 2.6,

$$
{ }_{2} F_{1}\left(\frac{1}{3}, \frac{2}{3} ; 1 ; \beta\right)=a\left(q^{2}\right) .
$$

By Lemma 5.5, (5.19), and (5.6),

$$
\begin{aligned}
\left(1+p+p^{2}\right){ }_{2} F_{1}\left(\frac{1}{2}, \frac{1}{2} ; 1 ; \alpha\right) & =3 \frac{a\left(q^{2}\right) c\left(q^{2}\right) \varphi^{2}\left(-q^{3}\right)}{c^{2}(q)} \\
& =\frac{\varphi(-q)}{\varphi\left(-q^{3}\right)} a\left(q^{2}\right) \\
& =\sqrt{1+2 p} a\left(q^{2}\right) \\
& =\sqrt{1+2 p}{ }_{2} F_{1}\left(\frac{1}{3}, \frac{2}{3} ; 1 ; \beta\right)
\end{aligned}
$$

by $(5.20)$.

Lastly, we show that our proof above of (5.17) is valid for $0<p<1$.

Observe that

$$
\frac{d \alpha}{d p}=\frac{6 p^{2}(1+p)^{2}}{(1+2 p)^{2}} \quad \text { and } \quad \frac{d \beta}{d p}=\frac{27 p\left(1-p^{2}\right)(1+2 p)(2+p)}{4\left(1+p+p^{2}\right)^{4}} .
$$

Hence, $\alpha(p)$ and $\beta(p)$ are monotonically increasing on $(0,1)$. Since $\alpha(0)=0=$ $\beta(0)$ and $\alpha(1)=1=\beta(1)$, it follows that Theorem 5.6 is valid for $0<p<1$.

We now prove a corresponding theorem with $\alpha$ and $\beta$ replaced by $1-\alpha$ and $1-\beta$, respectively.

Corollary 5.7. Let $\alpha$ and $\beta$ be as defined in Theorem 5.6. Then, for $0<p \leq$ 1 ,

$$
\text { (5.21) }\left(1+p+p^{2}\right)_{2} F_{1}\left(\frac{1}{2}, \frac{1}{2} ; 1 ; 1-\alpha\right)=\sqrt{3+6 p_{2}} F_{1}\left(\frac{1}{3}, \frac{2}{3} ; 1 ; 1-\beta\right) \text {. }
$$

Proof. By (5.19) and (5.20), with $q$ replaced by $-q$,

$$
\frac{a\left(q^{2}\right)}{\varphi^{2}\left(q^{3}\right)}=\frac{{ }_{2} F_{1}\left(\frac{1}{3}, \frac{2}{3} ; 1 ; \frac{c^{3}\left(q^{2}\right)}{a^{3}\left(q^{2}\right)}\right)}{{ }_{2} F_{1}\left(\frac{1}{2}, \frac{1}{2} ; 1 ; 1-\frac{\varphi^{4}\left(-q^{3}\right)}{\varphi^{4}\left(q^{3}\right)}\right)} .
$$

Thus, by (5.21) and (5.22), it suffices to prove that

$$
\frac{a\left(q^{2}\right)}{\varphi^{2}\left(q^{3}\right)}=\sqrt{3} \frac{{ }_{2} F_{1}\left(\frac{1}{3}, \frac{2}{3} ; 1 ; 1-\frac{c^{3}\left(q^{2}\right)}{a^{3}\left(q^{2}\right)}\right)}{{ }_{2} F_{1}\left(\frac{1}{2}, \frac{1}{2} ; 1 ; \frac{\varphi^{4}\left(-q^{3}\right)}{\varphi^{4}\left(q^{3}\right)}\right)} .
$$

By Lemma 2.9,

$$
q^{2}=\exp \left(-\frac{2 \pi}{\sqrt{3}} \frac{2 F_{1}\left(\frac{1}{3}, \frac{2}{3} ; 1 ; 1-\frac{c^{3}\left(q^{2}\right)}{a^{3}\left(q^{2}\right)}\right)}{{ }_{2} F_{1}\left(\frac{1}{3}, \frac{2}{3} ; 1 ; \frac{c^{3}\left(q^{2}\right)}{a^{3}\left(q^{2}\right)}\right)}\right)
$$


and by Entry 5 of Chapter 17 in Ramanujan's second notebook [5, p. 100],

$$
q^{3}=\exp \left(-\pi \frac{{ }_{2} F_{1}\left(\frac{1}{2}, \frac{1}{2} ; 1 ; \frac{\varphi^{4}\left(-q^{3}\right)}{\varphi^{4}\left(q^{3}\right)}\right)}{{ }_{2} F_{1}\left(\frac{1}{2}, \frac{1}{2} ; 1 ; 1-\frac{\varphi^{4}\left(-q^{3}\right)}{\varphi^{4}\left(q^{3}\right)}\right)}\right) .
$$

Combining (5.24) and (5.25), we find that

$$
\sqrt{3} \frac{{ }_{2} F_{1}\left(\frac{1}{3}, \frac{2}{3} ; 1 ; 1-\frac{c^{3}\left(q^{2}\right)}{a^{3}\left(q^{2}\right)}\right)}{{ }_{2} F_{1}\left(\frac{1}{3}, \frac{2}{3} ; 1 ; \frac{c^{3}\left(q^{2}\right)}{a^{3}\left(q^{2}\right)}\right)}=\frac{{ }_{2} F_{1}\left(\frac{1}{2}, \frac{1}{2} ; 1 ; \frac{\varphi^{4}\left(-q^{3}\right)}{\varphi^{4}\left(q^{3}\right)}\right)}{{ }_{2} F_{1}\left(\frac{1}{2}, \frac{1}{2} ; 1 ; 1-\frac{\varphi^{4}\left(-q^{3}\right)}{\varphi^{4}\left(q^{3}\right)}\right)} .
$$

We now see that (5.23) follows from combining (5.22) and (5.26).

Corollary 5.8. With $\alpha$ and $\beta$ as above, for $0<p<1$,

$$
\frac{{ }_{2} F_{1}\left(\frac{1}{2}, \frac{1}{2} ; 1 ; 1-\alpha\right)}{\sqrt{3}{ }_{2} F_{1}\left(\frac{1}{2}, \frac{1}{2} ; 1 ; \alpha\right)}=\frac{{ }_{2} F_{1}\left(\frac{1}{3}, \frac{2}{3} ; 1 ; 1-\beta\right)}{{ }_{2} F_{1}\left(\frac{1}{3}, \frac{2}{3} ; 1 ; \beta\right)} .
$$

Proof. Divide (5.21) by (5.17).

The authors' first proof of Corollary 5.7 employed Theorem 5.6 and a lemma arising from the hypergeometric differential equation satisfied by ${ }_{2} F_{1}(a, b ; c ; x)$ and ${ }_{2} F_{1}(a, b ; c ; 1-x)$. We are grateful to Heng Huat Chan for providing the proof that is given above.

Corollary 5.8 is important, for from (5.27) and (1.7),

$$
q_{3}^{3}=: q_{3}^{3}(\beta)=q^{2}(\alpha):=q^{2},
$$

where $q=q(\alpha)$ denotes the classical base. Thus, from Theorem 5.6 and (5.28), we can deduce the following theorem.

Theorem 5.9 (Transfer Principle). Suppose that we have a formula

$$
\Omega\left(q^{2}(\alpha), z(2 ; \alpha(p))\right)=0
$$

in the classical situation. Then

$$
\Omega\left(q_{3}^{3}(\beta), \frac{\sqrt{1+2 p}}{1+p+p^{2}} z(3 ; \beta(p))\right)=0,
$$

in the theory of signature 3.

If the formula (5.29) involves $\alpha$, in addition to its appearance through $q$ and $z$, it may be possible to convert (5.29) into a formula (5.30) involving only $\beta, q_{3}$, and $z(3)$. This good fortune is manifest in the next three instances, as we offer alternative proofs of Corollary 3.5, Theorem 4.4, and Theorem 4.5.

Second proof of Corollary 3.5. By elementary calculations,

(5.31) $1-\alpha=\frac{(1-p)(1+p)^{3}}{1+2 p} \quad$ and $\quad 1-\beta=\frac{(2+p)^{2}(1+2 p)^{2}(1-p)^{2}}{4\left(1+p+p^{2}\right)^{3}}$. 
From Entry 12(iii) of Chapter 17 [5, p. 124], Theorem 5.6, (5.28), and (5.30),

$$
\begin{aligned}
f\left(-q_{3}^{3}\right) & =f\left(-q^{2}\right)=\sqrt{z} 2^{-\frac{1}{3}}\{\alpha(1-\alpha) / q\}^{\frac{1}{12}} \\
& =\frac{(1+2 p)^{\frac{1}{4}}}{\left(1+p+p^{2}\right)^{\frac{1}{2}}} \sqrt{z(3)} 2^{-\frac{1}{3}}\left(\frac{p^{3}(2+p)(1-p)(1+p)^{3}}{(1+2 p)^{2}}\right)^{\frac{1}{12}} \frac{1}{q_{3}^{\frac{1}{8}}} \\
& =\frac{\sqrt{z(3)}}{q_{3}^{\frac{1}{8}} 3^{\frac{3}{8}}}\left(\frac{27 p^{2}(1+p)^{2}}{4\left(1+p+p^{2}\right)^{3}}\right)^{\frac{1}{8}}\left(\frac{(2+p)^{2}(1+2 p)^{2}(1-p)^{2}}{4\left(1+p+p^{2}\right)^{3}}\right)^{\frac{1}{24}} \\
& =\frac{\sqrt{z(3)}}{q_{3}^{\frac{1}{8}} 3^{\frac{3}{8}}} \beta^{\frac{1}{8}}(1-\beta)^{\frac{1}{24}},
\end{aligned}
$$

by Theorem 5.6 and (5.31). This completes our second proof of Corollary 3.5. Second proof of Theorem 4.4. By Entry 13(i) of Chapter 17 [5, p. 126], Theorem 5.6, (5.28), and (5.30),

$$
\begin{aligned}
M\left(q_{3}^{3}\right) & =M\left(q^{2}\right)=z^{4}\left(1-\alpha+\alpha^{2}\right) \\
& =\frac{(1+2 p)^{2}}{\left(1+p+p^{2}\right)^{4}} z^{4}(3)\left(1-\frac{p^{3}(2+p)}{1+2 p}+\frac{p^{6}(2+p)^{2}}{(1+2 p)^{2}}\right) \\
& =z^{4}(3) \frac{1+3 p-5 p^{3}+3 p^{5}+p^{6}}{\left(1+p+p^{2}\right)^{3}} \\
& =z^{4}(3) \frac{\left\{\left(1+p+p^{2}\right)^{3}-6 p^{2}(1+p)^{2}\right\}}{\left(1+p+p^{2}\right)^{3}} \\
& =z^{4}(3)\left(1-\frac{8}{9} \beta\right) .
\end{aligned}
$$

Second proof of Theorem 4.5. By Entry 13(ii) of Chapter 17 [5, p. 126], Theorem $5.6,(5.28)$, and (5.30),

$$
\begin{aligned}
N\left(q_{3}^{3}\right)= & N\left(q^{2}\right)=z^{6}(1+\alpha)\left(1-\frac{1}{2} \alpha\right)(1-2 \alpha) \\
= & \frac{z^{6}(3)}{2\left(1+p+p^{2}\right)^{6}}\left(1+2 p+p^{3}(2+p)\right)\left(2(1+2 p)-p^{3}(2+p)\right) \\
& \cdot\left(1+2 p-2 p^{3}(2+p)\right) \\
= & \frac{z^{6}(3)}{2\left(1+p+p^{2}\right)^{6}}\left\{2\left(1+p+p^{2}\right)^{6}-18 p^{2}(1+p)^{2}\left(1+p+p^{2}\right)^{3}\right. \\
= & z^{6}(3)\left(1-\frac{4}{3} \beta+\frac{8}{27} \beta^{2}\right) .
\end{aligned}
$$

Having thus proved Corollary 3.5, Theorem 4.4, and Theorem 4.5, we may use the process of trimidiation to reprove Corollary 3.4, Theorem 4.2, and Theorem 4.3.

6. MORE HIGHER ORDER TRANSFORMATIONS FOR HYPERGEOMETRIC SERIES

The first theorem will be used to prove Ramanujan's modular equations of degree 2 in the theory of signature 3 . 
Theorem 6.1 (p. 258). If

$$
\alpha:=\alpha(p):=\frac{p(3+p)^{2}}{2(1+p)^{3}} \quad \text { and } \quad \beta:=\beta(p):=\frac{p^{2}(3+p)}{4},
$$

then, for $0 \leq p<1$,

$$
{ }_{2} F_{1}\left(\frac{1}{3}, \frac{2}{3} ; 1 ; \alpha\right)=(1+p){ }_{2} F_{1}\left(\frac{1}{3}, \frac{2}{3} ; 1 ; \beta\right) .
$$

Proof. We first prove that

$$
a(q)-a\left(q^{2}\right)=2 \frac{c^{2}\left(q^{2}\right)}{c(q)} .
$$

From Entry 3(i),(ii) in Chapter 21 of Ramanujan's second notebook [5, p. 460],

$$
a\left(q^{2}\right)=\frac{\varphi^{3}(q)}{4 \varphi\left(q^{3}\right)}+\frac{3 \varphi^{3}\left(q^{3}\right)}{4 \varphi(q)} .
$$

Thus, by (6.4), Theorem 2.13, (1.17), (2.13), (2.14), (2.15), (5.10), and (5.7),

$$
\begin{aligned}
a(q)-a\left(q^{2}\right) & =4 \frac{\psi^{3}\left(q^{2}\right)}{\psi\left(q^{6}\right)}-\frac{15 \varphi^{3}\left(q^{3}\right)}{4 \varphi(q)}-\frac{\varphi^{3}(q)}{4 \varphi\left(q^{3}\right)} \\
& =\frac{z_{1}^{\frac{3}{2}}}{z_{3}^{\frac{1}{2}}}\left(\frac{\alpha^{3}}{\beta}\right)^{\frac{1}{4}}-\frac{15}{4} \frac{z_{3}^{\frac{3}{2}}}{z_{1}^{\frac{1}{2}}}-\frac{z_{1}^{\frac{3}{2}}}{4 z_{3}^{\frac{1}{2}}} \\
& =\frac{z_{3}^{\frac{3}{2}}}{z_{1}^{\frac{1}{2}}}\left(m^{2} \frac{(3+m)^{2}}{4 m^{2}}-\frac{15}{4}-\frac{1}{4} m^{2}\right) \\
& =\frac{3 z_{3}^{\frac{3}{2}}}{2 z_{1}^{\frac{1}{2}}}(m-1) \\
& =3 \frac{z_{3}^{\frac{3}{2}}}{z_{1}^{\frac{1}{2}}}\left(\frac{\beta^{3}}{\alpha}\right)^{\frac{1}{8}} \\
& =6 q \frac{\psi^{3}\left(q^{3}\right)}{\psi(q)} \\
& =2 \frac{c^{2}\left(q^{2}\right)}{c(q)}
\end{aligned}
$$

which completes the proof of $(6.3)$.

Secondly, we prove that

$$
a(q)+2 a\left(q^{2}\right)=\frac{c^{2}(q)}{c\left(q^{2}\right)} .
$$

By (6.4), Theorem 2.13, (1.17), (2.13), (2.14), (2.15), (3.10), (3.11), (5.9), 
and (5.6),

$$
\begin{aligned}
a(q)+2 a\left(q^{2}\right) & =4 \frac{\psi^{3}\left(q^{2}\right)}{\psi\left(q^{6}\right)}-\frac{3 \varphi^{3}\left(q^{3}\right)}{2 \varphi(q)}+\frac{\varphi^{3}(q)}{2 \varphi\left(q^{3}\right)} \\
& =\frac{z_{1}^{\frac{3}{2}}}{z_{3}^{\frac{1}{2}}}\left(\frac{\alpha^{3}}{\beta}\right)^{\frac{1}{4}}-\frac{3}{2} \frac{z_{3}^{\frac{3}{2}}}{z_{1}^{\frac{1}{2}}}+\frac{z_{1}^{\frac{3}{2}}}{2 z_{3}^{\frac{1}{2}}} \\
& =\frac{z_{3}^{\frac{3}{2}}}{z_{1}^{\frac{1}{2}}}\left(\frac{(3+m)^{2}}{4}-\frac{3}{2}+\frac{1}{2} m^{2}\right) \\
& =\frac{3 z_{3}^{\frac{3}{2}}}{4 z_{1}^{\frac{1}{2}}}(m+1)^{2} \\
& =3 \frac{z_{3}^{\frac{3}{2}}}{z_{1}^{\frac{1}{2}}}\left(\frac{(1-\beta)^{3}}{1-\alpha}\right)^{\frac{1}{4}} \\
& =3 \frac{\varphi^{3}\left(-q^{3}\right)}{\varphi(-q)} \\
& =\frac{c^{2}(q)}{c\left(q^{2}\right)}
\end{aligned}
$$

which proves $(6.5)$.

Now let

$$
p:=p(q):=\frac{a(q)}{a\left(q^{2}\right)}-1 \text {. }
$$

(Note that $p$ tends to 0 as $q$ tends to 0 , by (2.2).) Then by (6.6), (6.3), and (6.5),

$$
\begin{aligned}
\alpha=\frac{p(3+p)^{2}}{2(1+p)^{3}} & =\frac{1}{2}\left(\frac{a(q)}{a\left(q^{2}\right)}-1\right)\left(\frac{a(q)}{a\left(q^{2}\right)}+2\right)^{2} \frac{a^{3}\left(q^{2}\right)}{a^{3}(q)} \\
& =\frac{\left(a(q)-a\left(q^{2}\right)\right)\left(a(q)+2 a\left(q^{2}\right)\right)^{2}}{2 a^{3}(q)} \\
& =2 \frac{c^{2}\left(q^{2}\right)}{c(q)} \frac{c^{4}(q)}{c^{2}\left(q^{2}\right)} \frac{1}{2 a^{3}(q)} \\
& =\frac{c^{3}(q)}{a^{3}(q)} .
\end{aligned}
$$

Also, by (6.6), (6.3), and (6.5),

$$
\begin{aligned}
\beta=\frac{p^{2}(3+p)}{4} & =\frac{1}{4}\left(\frac{a(q)}{a\left(q^{2}\right)}-1\right)^{2}\left(\frac{a(q)}{a\left(q^{2}\right)}+2\right) \\
& =\frac{1}{4 a^{3}\left(q^{2}\right)} \frac{4 c^{4}\left(q^{2}\right)}{c^{2}(q)} \frac{c^{2}(q)}{c\left(q^{2}\right)} \\
& =\frac{c^{3}\left(q^{2}\right)}{a^{3}\left(q^{2}\right)} .
\end{aligned}
$$

The desired result now follows immediately from Lemma 2.6, (6.6), (6.7), and (6.8). 
We now determine those values of $p$ for which our proof of (6.2) above holds.

By (6.1),

$$
\frac{d \alpha}{d p}=\frac{3(3+p)(1-p)}{2(1+p)^{4}} \quad \text { and } \quad \frac{d \beta}{d p}=\frac{3 p(2+p)}{4}
$$

Thus, $\alpha(p)$ and $\beta(p)$ are monotonically increasing on $(0,1)$. Since $\alpha(0)=0=$ $\beta(0)$ and $\alpha(1)=1=\beta(1)$, it follows that (6.2) holds for $0 \leq p<1$.

As functions of $p$, the left and right sides of (6.2) are solutions of the differential equation, $p(1-p)(1+p)^{2}(2+p)(3+p) u^{\prime \prime}+2(1+p)\left(3-4 p-6 p^{2}-4 p^{3}-p^{4}\right) u^{\prime}=2(1-p)(3+p) u$.

Corollary 6.2. Let $\alpha$ and $\beta$ be defined by (6.1). Then, for $0<p \leq 1$,

$$
{ }_{2} F_{1}\left(\frac{1}{3}, \frac{2}{3} ; 1 ; 1-\alpha\right)=\frac{1}{2}(1+p){ }_{2} F_{1}\left(\frac{1}{3}, \frac{2}{3} ; 1 ; 1-\beta\right) .
$$

Proof. By Lemma 2.9 and (6.7) and (6.8), respectively,

$$
q=\exp \left(-\frac{2 \pi}{\sqrt{3}} \frac{{ }_{2} F_{1}\left(\frac{1}{3}, \frac{2}{3} ; 1 ; 1-\alpha\right)}{{ }_{2} F_{1}\left(\frac{1}{3}, \frac{2}{3} ; 1 ; \alpha\right)}\right)
$$

and

$$
q^{2}=\exp \left(-\frac{2 \pi}{\sqrt{3}} \frac{{ }_{2} F_{1}\left(\frac{1}{3}, \frac{2}{3} ; 1 ; 1-\beta\right)}{{ }_{2} F_{1}\left(\frac{1}{3}, \frac{2}{3} ; 1 ; \beta\right)}\right) .
$$

The desired result now follows easily from (6.2), (6.10), and (6.11).

Corollary 6.3. Let $\alpha(p)$ and $\beta(p)$ be given by (6.1). Then, for $0<p<1$,

$$
2 \frac{{ }_{2} F_{1}\left(\frac{1}{3}, \frac{2}{3} ; 1 ; 1-\alpha\right)}{{ }_{2} F_{1}\left(\frac{1}{3}, \frac{2}{3} ; 1 ; \alpha\right)}=\frac{{ }_{2} F_{1}\left(\frac{1}{3}, \frac{2}{3} ; 1 ; 1-\beta\right)}{{ }_{2} F_{1}\left(\frac{1}{3}, \frac{2}{3} ; 1 ; \beta\right)}
$$

and

$$
m(3)=1+p
$$

where $m(3)$ is the multiplier of degree 2 for the theory of signature 3.

Proof. Divide (6.9) by (6.2).

Since (6.12) is the defining relation for a modular equation of degree 2 in the theory of signature $3,(6.13)$ follows from (1.10) and (6.2).

The next transformation is useful in establishing Ramanujan's modular equation of degree 4 .

Theorem 6.4 (p. 258). Let

$$
\alpha:=\alpha(p):=\frac{27 p(1+p)^{4}}{2\left(1+4 p+p^{2}\right)^{3}} \quad \text { and } \quad \beta:=\beta(p):=\frac{27 p^{4}(1+p)}{2\left(2+2 p-p^{2}\right)^{3}} \text {. }
$$

Then, for $0 \leq p<1$,

$$
\left(2+2 p-p^{2}\right)_{2} F_{1}\left(\frac{1}{3}, \frac{2}{3} ; 1 ; \alpha\right)=2\left(1+4 p+p^{2}\right){ }_{2} F_{1}\left(\frac{1}{3}, \frac{2}{3} ; 1 ; \beta\right) \text {. }
$$


Proof. For brevity, set $z(x)={ }_{2} F_{1}\left(\frac{1}{3}, \frac{2}{3} ; 1 ; x\right)$. In view of Theorem 6.1, we want to find $x$ and $y$ so that

$$
\begin{aligned}
z\left(\frac{y(3+y)^{2}}{2(1+y)^{3}}\right) & =(1+y) z\left(\frac{y^{2}(3+y)}{4}\right) \\
& =(1+y) z\left(\frac{x(3+x)^{2}}{2(1+x)^{3}}\right) \\
& =(1+y)(1+x) z\left(\frac{x^{2}(3+x)}{4}\right),
\end{aligned}
$$

$$
\begin{aligned}
& \frac{y(3+y)^{2}}{2(1+y)^{3}}=\frac{27 p(1+p)^{4}}{2\left(1+4 p+p^{2}\right)^{3}} \\
& \frac{x^{2}(3+x)}{4}=\frac{27 p^{4}(1+p)}{2\left(2+2 p-p^{2}\right)^{3}}
\end{aligned}
$$

and

$$
(1+y)(1+x)=\frac{2\left(1+4 p+p^{2}\right)}{2+2 p-p^{2}} .
$$

Solving (6.18) for $x$, or judiciously guessing the solution with the help of (6.19), we find that

$$
x=\frac{3 p^{2}}{2+2 p-p^{2}} .
$$

An elementary calculation shows that (6.18) then holds. Substituting (6.20) into (6.19) and solving for $y$, we find that

$$
y=\frac{3 p}{1+p+p^{2}} \text {. }
$$

Substituting (6.21) into the left side of (6.17), we see that, indeed, (6.17) holds. Lastly, it is easily checked that with $x$ and $y$ as chosen above,

$$
\frac{x(3+x)^{2}}{2(1+x)^{3}}=\frac{y^{2}(3+y)}{4}
$$

i.e., the middle equality of (6.16) holds. Hence, $(6.15)$ is valid, and the interval of validity, $0 \leq p<1$, follows by an elementary argument like that in the proof of Theorem 6.1.

Corollary 6.5. Let $\alpha$ and $\beta$ be defined by (6.14). Then, for $0<p \leq 1$, (6.22)

$$
2\left(2+2 p-p^{2}\right){ }_{2} F_{1}\left(\frac{1}{3}, \frac{2}{3} ; 1 ; 1-\alpha\right)=\left(1+4 p+p^{2}\right){ }_{2} F_{1}\left(\frac{1}{3}, \frac{2}{3} ; 1 ; 1-\beta\right) .
$$

Proof. The proof is analogous to that for Corollary 6.2.

Corollary 6.6. Let $\alpha$ and $\beta$ be defined by (6.14). Then, for $0<p<1$,

$$
4 \frac{{ }_{2} F_{1}\left(\frac{1}{3}, \frac{2}{3} ; 1 ; 1-\alpha\right)}{{ }_{2} F_{1}\left(\frac{1}{3}, \frac{2}{3} ; 1 ; \alpha\right)}=\frac{{ }_{2} F_{1}\left(\frac{1}{3}, \frac{2}{3} ; 1 ; 1-\beta\right)}{{ }_{2} F_{1}\left(\frac{1}{3}, \frac{2}{3} ; 1 ; \beta\right)} .
$$

Proof. Divide (6.22) by (6.15). 


\section{Modular equations in the Theory of Signature 3}

We first show that Corollary 6.3 can be utilized to prove five modular equations of degree 2 offered by Ramanujan.

Theorem 7.1 (p. 259). If $\beta$ has degree 2 in the theory of signature 3 , then

$$
\begin{gathered}
(\alpha \beta)^{\frac{1}{3}}+\{(1-\alpha)(1-\beta)\}^{\frac{1}{3}}=1, \\
\left(\frac{\alpha^{2}}{\beta}\right)^{\frac{1}{3}}-\left\{\frac{(1-\alpha)^{2}}{1-\beta}\right\}^{\frac{1}{3}}=\frac{2}{1+p}=\frac{2}{m},
\end{gathered}
$$

$$
\left\{\frac{(1-\beta)^{2}}{1-\alpha}\right\}^{\frac{1}{3}}-\left(\frac{\beta^{2}}{\alpha}\right)^{\frac{1}{3}}=m
$$

$$
\left(\frac{\alpha^{2}}{\beta}\right)^{\frac{1}{3}}+\left\{\frac{(1-\alpha)^{2}}{1-\beta}\right\}^{\frac{1}{3}}=\frac{4}{m^{2}},
$$

and

$$
\left\{\frac{(1-\beta)^{2}}{1-\alpha}\right\}^{\frac{1}{3}}+\left(\frac{\beta^{2}}{\alpha}\right)^{\frac{1}{3}}=m^{2}
$$

Proof. From (6.1), by elementary calculations,

$$
1-\alpha=\frac{(1-p)^{2}(2+p)}{2(1+p)^{3}} \quad \text { and } \quad 1-\beta=\frac{(1-p)(2+p)^{2}}{4} .
$$

Thus, from (6.1) and (7.1), respectively,

$$
(\alpha \beta)^{\frac{1}{3}}=\frac{p(3+p)}{2(1+p)} \quad \text { and } \quad\{(1-\alpha)(1-\beta)\}^{\frac{1}{3}}=\frac{(1-p)(2+p)}{2(1+p)} .
$$

Hence,

$$
(\alpha \beta)^{\frac{1}{3}}+\{(1-\alpha)(1-\beta)\}^{\frac{1}{3}}=\frac{3 p+p^{2}+2-p-p^{2}}{2(1+p)}=\frac{2 p+2}{2(1+p)}=1 .
$$

Similarly, by (6.1), (7.1), and (6.13),

$$
\begin{gathered}
\left(\frac{\alpha^{2}}{\beta}\right)^{\frac{1}{3}}-\left\{\frac{(1-\alpha)^{2}}{1-\beta}\right\}^{\frac{1}{3}}=\frac{3+p}{(1+p)^{2}}-\frac{1-p}{(1+p)^{2}}=\frac{2}{1+p}=\frac{2}{m} \\
\left\{\frac{(1-\beta)^{2}}{1-\alpha}\right\}^{\frac{1}{3}}-\left(\frac{\beta^{2}}{\alpha}\right)^{\frac{1}{3}}=\frac{(2+p)(1+p)}{2}-\frac{p(1+p)}{2}=1+p=m \\
\left(\frac{\alpha^{2}}{\beta}\right)^{\frac{1}{3}}+\left\{\frac{(1-\alpha)^{2}}{1-\beta}\right\}^{\frac{1}{3}}=\frac{3+p}{(1+p)^{2}}+\frac{1-p}{(1+p)^{2}}=\frac{4}{(1+p)^{2}}=\frac{4}{m^{2}}
\end{gathered}
$$

and

$$
\left\{\frac{(1-\beta)^{2}}{1-\alpha}\right\}^{\frac{1}{3}}+\left(\frac{\beta^{2}}{\alpha}\right)^{\frac{1}{3}}=\frac{(2+p)(1+p)}{2}+\frac{p(1+p)}{2}=(1+p)^{2}=m^{2} .
$$

Thus, the proofs of (i)-(v) have been completed. 
Theorem 7.2 (p. 259). Let $\beta$ be of degree 4 , and let $m$ be the associated multiplier in the theory of signature 3 . Then

$$
m=\left(\frac{\beta}{\alpha}\right)^{\frac{1}{3}}+\left(\frac{1-\beta}{1-\alpha}\right)^{\frac{1}{3}}-\frac{4}{m}\left(\frac{\beta(1-\beta)}{\alpha(1-\alpha)}\right)^{\frac{1}{3}} .
$$

Proof. From (6.14), we easily find that

$$
1-\alpha=\frac{(1-p)^{4}(2+p)(1+2 p)}{2\left(1+4 p+p^{2}\right)^{3}} \quad \text { and } \quad 1-\beta=\frac{(1-p)(2+p)^{4}(1+2 p)}{2\left(2+2 p-p^{2}\right)^{3}} \text {. }
$$

Thus, from (6.14) and (7.2),

$$
\begin{aligned}
\left(\frac{\beta}{\alpha}\right)^{\frac{1}{3}}+\left(\frac{1-\beta}{1-\alpha}\right)^{\frac{1}{3}} & =\frac{p\left(1+4 p+p^{2}\right)}{(1+p)\left(2+2 p-p^{2}\right)}+\frac{(2+p)\left(1+4 p+p^{2}\right)}{(1-p)\left(2+2 p-p^{2}\right)} \\
& =\frac{2\left(1+4 p+p^{2}\right)(1+2 p)}{\left(2+2 p-p^{2}\right)(1+p)(1-p)}
\end{aligned}
$$

From Theorem 6.4,

$$
m=\frac{2\left(1+4 p+p^{2}\right)}{2+2 p-p^{2}}
$$

Hence, by (7.2) and (7.4),

$$
\begin{aligned}
\frac{4}{m}\left(\frac{\beta(1-\beta)}{\alpha(1-\alpha)}\right)^{\frac{1}{3}} & =\frac{2\left(2+2 p-p^{2}\right)}{1+4 p+p^{2}} \frac{p(2+p)\left(1+4 p+p^{2}\right)^{2}}{(1+p)(1-p)\left(2+2 p-p^{2}\right)^{2}} \\
& =\frac{2 p(2+p)\left(1+4 p+p^{2}\right)}{(1+p)(1-p)\left(2+2 p-p^{2}\right)} .
\end{aligned}
$$

Therefore, combining (7.3) and (7.5), we deduce that

$$
\begin{aligned}
\left(\frac{\beta}{\alpha}\right)^{\frac{1}{3}} & +\left(\frac{1-\beta}{1-\alpha}\right)^{\frac{1}{3}}-\frac{4}{m}\left(\frac{\beta(1-\beta)}{\alpha(1-\alpha)}\right)^{\frac{1}{3}} \\
& =\frac{2\left(1+4 p+p^{2}\right)(1+2 p)}{\left(2+2 p-p^{2}\right)(1+p)(1-p)}-\frac{2 p(2+p)\left(1+4 p+p^{2}\right)}{(1+p)(1-p)\left(2+2 p-p^{2}\right)} \\
& =\frac{2\left(1+4 p+p^{2}\right)}{2+2 p-p^{2}}=m,
\end{aligned}
$$

by (7.4), and the proof is complete.

Theorem 7.3 (p. 204, first notebook). Let $\alpha, \beta$, and $\gamma$ have degrees 1, 2, and 4 , respectively. Let $m_{1}$ and $m_{2}$ denote the multipliers associated with the pairs $\alpha, \beta$ and $\beta, \gamma$, respectively. Then

$$
\frac{\sqrt{3}\{\beta(1-\beta)\}^{\frac{1}{6}}}{\{\alpha(1-\gamma)\}^{\frac{1}{3}}-\{\gamma(1-\alpha)\}^{\frac{1}{3}}}=\frac{m_{1}}{m_{2}} .
$$

Proof. In (6.14), replace $\beta$ by $\gamma$, so that for $0 \leq p<1$,

$$
\alpha=\frac{27 p(1+p)^{4}}{2\left(1+4 p+p^{2}\right)^{3}} \quad \text { and } \quad \gamma=\frac{27 p^{4}(1+p)}{2\left(2+2 p-p^{2}\right)^{3}} .
$$

From the proof of Theorem $6.4, \beta$ has the representations

$$
\frac{y^{2}(3+y)}{4} \text { and } \frac{x(3+x)^{2}}{2(1+x)^{3}}
$$


where $x$ and $y$ are given by (6.20) and (6.21), respectively. In either case, a short calculation shows that

$$
\beta=\frac{27 p^{2}(1+p)^{2}}{4\left(1+p+p^{2}\right)^{3}} .
$$

Using (7.6) and (7.7), we find that

$$
\begin{aligned}
1-\alpha & =\frac{2 p^{6}-3 p^{5}-6 p^{4}+14 p^{3}-6 p^{2}-3 p+2}{2\left(1+4 p+p^{2}\right)^{3}} \\
& =\frac{(p-1)^{4}\left(2 p^{2}+5 p+2\right)}{2\left(1+4 p+p^{2}\right)^{3}}, \\
1-\beta & =\frac{4 p^{6}+12 p^{5}-3 p^{4}-26 p^{3}-3 p^{2}+12 p+4}{4\left(1+p+p^{2}\right)^{3}} \\
& =\frac{(p-1)^{2}\left(2 p^{2}+5 p+2\right)^{2}}{4\left(1+p+p^{2}\right)^{3}},
\end{aligned}
$$

and

$$
\begin{aligned}
1-\gamma & =\frac{-2 p^{6}-15 p^{5}-39 p^{4}-32 p^{3}+24 p^{2}+48 p+16}{2\left(2+2 p-p^{2}\right)^{3}} \\
& =-\frac{(p-1)(p+2)^{3}\left(2 p^{2}+5 p+2\right)}{2\left(2+2 p-p^{2}\right)^{3}}
\end{aligned}
$$

Hence, from (7.6)-(7.10),

$$
\begin{aligned}
\frac{\sqrt{3}\{\beta(1-\beta)\}^{\frac{1}{6}}}{\{\alpha(1-\gamma)\}^{\frac{1}{3}}-\{\gamma(1-\alpha)\}^{\frac{1}{3}}} & \frac{\sqrt{3}\left(\frac{27 p^{2}(1+p)^{2}}{4\left(1+p+p^{2}\right)^{3}} \frac{(p-1)^{2}\left(2 p^{2}+5 p+2\right)^{2}}{4\left(1+p+p^{2}\right)^{3}}\right)^{\frac{1}{6}}}{\left\{\left(\frac{27 p(1+p)^{4}}{2\left(1+4 p+p^{2}\right)^{3}} \frac{(1-p)(p+2)^{3}\left(2 p^{2}+5 p+2\right)}{2\left(2+2 p-p^{2}\right)^{3}}\right)^{\frac{1}{3}}\right.} \\
= & \left.\left.\frac{\left(1+4 p+p^{2}\right)\left(2+2 p-p^{2}\right)}{2\left(2+2 p-p^{2}\right)^{3}} \frac{(p-1)^{4}\left(2 p^{2}+5 p+2\right)}{2\left(1+4 p+p^{2}\right)^{3}}\right)^{\frac{1}{3}}\right\} \\
= & \frac{\left(1+4 p+p^{2}\right)\left(2+2 p-p^{2}\right)}{\left(1+p+p^{2}\right)\{(1+p)(2+p)-p(1-p)\}} \\
= & \frac{\left(1+p+p^{2}\right)^{2}}{2(1+4)}
\end{aligned}
$$

On the other hand, from the proof of Theorem 6.4, and from (6.20) and (6.21),

$$
\frac{m_{1}}{m_{2}}=\frac{1+y}{1+x}=\frac{\left(1+4 p+p^{2}\right)\left(2+2 p-p^{2}\right)}{2\left(1+p+p^{2}\right)^{2}} .
$$

Combining (7.11) and (7.12), we complete the proof.

Next, we show that Ramanujan's beautiful cubic transformation in Corollary 2.4 yields the defining relation for modular equations of degree 3 . We then iterate the transformation in order to derive Ramanujan's modular equation of degree 9 . 
Lemma 7.4. If

$$
\alpha=1-\left(\frac{1-\beta^{\frac{1}{3}}}{1+2 \beta^{\frac{1}{3}}}\right)^{3}
$$

then

$$
\frac{{ }_{2} F_{1}\left(\frac{1}{3}, \frac{2}{3} ; 1 ; 1-\beta\right)}{{ }_{2} F_{1}\left(\frac{1}{3}, \frac{2}{3} ; 1 ; \beta\right)}=3 \frac{{ }_{2} F_{1}\left(\frac{1}{3}, \frac{2}{3} ; 1 ; 1-\alpha\right)}{{ }_{2} F_{1}\left(\frac{1}{3}, \frac{2}{3} ; 1 ; \alpha\right)} .
$$

Furthermore, the multiplier $m$ is equal to $1+2 \beta^{\frac{1}{3}}$.

Proof. In (2.23) and (2.25), set $x=\beta^{\frac{1}{3}}$. Dividing (2.25) by (2.23), we deduce (7.14). The formula $m=1+2 \beta^{\frac{1}{3}}$ is an immediate consequence of (2.23).

Theorem 7.5 (p. 259). If $m$ is the multiplier for modular equations of degree 9 , then

$$
m=3 \frac{1+2 \beta^{\frac{1}{3}}}{1+2(1-\alpha)^{\frac{1}{3}}},
$$

where $\beta$ has degree 9.

Proof. Let $\alpha$ be given by (7.13), but with $\beta$ replaced by

$$
t:=1-\left(\frac{1-\beta^{\frac{1}{3}}}{1+2 \beta^{\frac{1}{3}}}\right)^{3} \text {. }
$$

Applying (2.23) twice, we find that

$$
\begin{aligned}
{ }_{2} F_{1}\left(\frac{1}{3}, \frac{2}{3} ; 1 ; \alpha\right) & =\left(1+2 t^{\frac{1}{3}}\right){ }_{2} F_{1}\left(\frac{1}{3}, \frac{2}{3} ; 1 ; t\right) \\
& =\left(1+2 t^{\frac{1}{3}}\right)\left(1+2 \beta^{\frac{1}{3}}\right){ }_{2} F_{1}\left(\frac{1}{3}, \frac{2}{3} ; 1 ; \beta\right) .
\end{aligned}
$$

We want to express the multiplier

$$
m=\left(1+2 t^{\frac{1}{3}}\right)\left(1+2 \beta^{\frac{1}{3}}\right)
$$

entirely in terms of $\alpha$ and $\beta$. Solving for $\beta^{\frac{1}{3}}$ in (7.13) and then replacing $\beta$ by $t$, we find that

$$
t^{\frac{1}{3}}=\frac{1-(1-\alpha)^{\frac{1}{3}}}{2(1-\alpha)^{\frac{1}{3}}+1} .
$$

Thus,

$$
1+2 t^{\frac{1}{3}}=\frac{3}{2(1-\alpha)^{\frac{1}{3}}+1} .
$$

Using this in (7.16), we deduce (7.15) to complete the proof.

Theorem 7.6 (p. 259). If $\beta$ has degree 5, then

$$
(\alpha \beta)^{\frac{1}{3}}+\{(1-\alpha)(1-\beta)\}^{\frac{1}{3}}+3\{\alpha \beta(1-\alpha)(1-\beta)\}^{\frac{1}{6}}=1 .
$$

Proof. By Corollary 3.2, we may rewrite (7.17) in the form

$$
b(q) b\left(q^{5}\right)+c(q) c\left(q^{5}\right)+3 \sqrt{b(q) c(q) b\left(q^{5}\right) c\left(q^{5}\right)}=a(q) a\left(q^{5}\right) .
$$


From Theorem 2.2 and (5.4) and (5.5) of Lemma 5.1, we find that

$$
a(q)=\left\{\frac{f^{12}(-q)+27 q f^{12}\left(-q^{3}\right)}{f^{3}(-q) f^{3}\left(-q^{3}\right)}\right\}^{\frac{1}{3}} .
$$

(In fact, (7.19) is given by Ramanujan in his second notebook [5, p. 460, Entry 3 (i)].) Thus, by (5.4), (5.5), and (7.19), (7.18) is equivalent to the eta-function identity

$$
\begin{gathered}
\frac{f^{3}(-q) f^{3}\left(-q^{5}\right)}{f\left(-q^{3}\right) f\left(-q^{15}\right)}+9 q^{2} \frac{f^{3}\left(-q^{3}\right) f^{3}\left(-q^{15}\right)}{f(-q) f\left(-q^{5}\right)}+9 q f(-q) f\left(-q^{3}\right) f\left(-q^{5}\right) f\left(-q^{15}\right) \\
=\frac{\left\{f^{12}(-q)+27 q f^{12}\left(-q^{3}\right)\right\}^{\frac{1}{3}}\left\{f^{12}\left(-q^{5}\right)+27 q^{5} f^{12}\left(-q^{15}\right)\right\}^{\frac{1}{3}}}{f(-q) f\left(-q^{3}\right) f\left(-q^{5}\right) f\left(-q^{15}\right)} .
\end{gathered}
$$

Cubing both sides of (7.20), simplifying, and setting

$$
A=f(-q), \quad B=f\left(-q^{3}\right), \quad C=f\left(-q^{5}\right), \quad \text { and } \quad D=f\left(-q^{15}\right),
$$

we deduce that (7.13) is equivalent to the proposed identity

$$
\begin{aligned}
45 q^{2} A^{6} B^{6} C^{6} D^{6}+10 q A^{8} C^{8} B^{4} D^{4}+90 q^{3} A^{4} C^{4} B^{8} D^{8} \\
+A^{10} C^{10} B^{2} D^{2}+81 q^{4} A^{2} C^{2} B^{10} D^{10}=q^{4} A^{12} D^{12}+B^{12} C^{12} .
\end{aligned}
$$

Setting

$$
P=\frac{f(-q)}{q^{\frac{1}{12}} f\left(-q^{3}\right)} \quad \text { and } \quad Q=\frac{f\left(-q^{5}\right)}{q^{\frac{5}{12}} f\left(-q^{15}\right)}
$$

and dividing both sides of $(7.21)$ by $q^{2}(A B C D)^{6}$, we find that (7.21) can be written in the equivalent form

$$
45+10 P^{2} Q^{2}+\frac{90}{P^{2} Q^{2}}+P^{4} Q^{4}+\frac{81}{P^{4} Q^{4}}=\frac{P^{6}}{Q^{6}}+\frac{Q^{6}}{P^{6}},
$$

or

$$
\left\{(P Q)^{2}+5+\frac{9}{(P Q)^{2}}\right\}^{2}=\left\{\left(\frac{Q}{P}\right)^{3}-\left(\frac{P}{Q}\right)^{3}\right\}^{2} .
$$

By examining $P$ and $Q$ in a neighborhood of $q=0$, so that the proper square root can be taken on the right side of (7.22), we find that (7.22) is equivalent to the identity

$$
(P Q)^{2}+5+\frac{9}{(P Q)^{2}}=\left(\frac{Q}{P}\right)^{3}-\left(\frac{P}{Q}\right)^{3} .
$$

Now (7.23) is stated by Ramanujan on page 324 of his second notebook [35] and has been proved by Berndt in his book [8, Chapter 25, Entry 62]. (See also a paper by Berndt and L.-C. Zhang [10], where several of Ramanujan's eta-function identities similar to (7.23) are proved.) This therefore completes the proof of (7.17).

Theorem 7.7 (p. 259). If $\beta$ has degree 7 , then

$$
m=\left(\frac{\beta}{\alpha}\right)^{\frac{1}{3}}+\left(\frac{1-\beta}{1-\alpha}\right)^{\frac{1}{3}}-\frac{7}{m}\left(\frac{\beta(1-\beta)}{\alpha(1-\alpha)}\right)^{\frac{1}{3}}-3\left(\frac{\beta(1-\beta)}{\alpha(1-\alpha)}\right)^{\frac{1}{6}} .
$$


Proof. Using Corollary 3.2 and recalling that $m=z_{1} / z_{7}$, we find that (7.24) is equivalent to the equality

$$
1=\frac{b\left(q^{7}\right)}{b(q)}+\frac{c\left(q^{7}\right)}{c(q)}-7 \frac{b\left(q^{7}\right) c\left(q^{7}\right)}{b(q) c(q)}-3 \sqrt{\frac{b\left(q^{7}\right) c\left(q^{7}\right)}{b(q) c(q)}}
$$

Employing (5.4) and (5.5) in (7.25) and then multiplying both sides of the resulting equality by $f(-q) f\left(-q^{3}\right) /\left(q f\left(-q^{7}\right) f\left(-q^{21}\right)\right)$, we find that (7.25) may be written in the equivalent form

$$
\begin{aligned}
\frac{f(-q) f\left(-q^{3}\right)}{q f\left(-q^{7}\right) f\left(-q^{21}\right)}= & \frac{q f^{2}(-q) f^{2}\left(-q^{21}\right)}{f^{2}\left(-q^{3}\right) f^{2}\left(-q^{7}\right)}+\frac{f^{2}\left(-q^{3}\right) f^{2}\left(-q^{7}\right)}{q f^{2}(-q) f^{2}\left(-q^{21}\right)} \\
& -7 \frac{q f\left(-q^{7}\right) f\left(-q^{21}\right)}{f(-q) f\left(-q^{3}\right)}-3 .
\end{aligned}
$$

If we set

$$
P=\frac{f(-q)}{q^{\frac{1}{4}} f\left(-q^{7}\right)} \quad \text { and } \quad Q=\frac{f\left(-q^{3}\right)}{q^{\frac{3}{4}} f\left(-q^{21}\right)},
$$

we deduce that (7.26) is equivalent to the identity

$$
P Q+\frac{7}{P Q}=\frac{P^{2}}{Q^{2}}+\frac{Q^{2}}{P^{2}}-3
$$

However, (7.27) can be found on page 323 of Ramanujan's second notebook [35] and has been proved by Berndt and Zhang [10, Theorem 4]. See also [8, Chapter 25, Entry 68]. This completes the proof of (7.24).

Theorem 7.8 (p. 259). If $\beta$ has degree 11 , then

$$
\begin{aligned}
& (\alpha \beta)^{\frac{1}{3}}+\{(1-\alpha)(1-\beta)\}^{\frac{1}{3}}+6\{\alpha \beta(1-\alpha)(1-\beta)\}^{\frac{1}{6}} \\
& \quad+3 \sqrt{3}\{\alpha \beta(1-\alpha)(1-\beta)\}^{\frac{1}{12}}\left\{(\alpha \beta)^{\frac{1}{6}}+\{(1-\alpha)(1-\beta)\}^{\frac{1}{6}}\right\}=1 .
\end{aligned}
$$

Proof. Employing Corollary 3.2, we find that (7.28) is equivalent to the identity

$$
\begin{aligned}
c(q) & c\left(q^{11}\right)+b(q) b\left(q^{11}\right)+6 \sqrt{b(q) b\left(q^{11}\right) c(q) c\left(q^{11}\right)} \\
& +3 \sqrt{3}\left\{b(q) b\left(q^{11}\right) c(q) c\left(q^{11}\right)\right\}^{\frac{1}{4}} \\
& \cdot\left\{\sqrt{c(q) c\left(q^{11}\right)}+\sqrt{b(q) b\left(q^{11}\right)}\right\}=a(q) a\left(q^{11}\right) .
\end{aligned}
$$

By (5.4), (5.5), and (7.19), (7.29) can be transformed into the equivalent identity

$$
\begin{aligned}
& 9 \frac{q^{4} f^{3}\left(-q^{3}\right) f^{3}\left(-q^{33}\right)}{f(-q) f\left(-q^{11}\right)}+\frac{f^{3}(-q) f^{3}\left(-q^{11}\right)}{f\left(-q^{3}\right) f\left(-q^{33}\right)} \\
& +18 q^{2} f(-q) f\left(-q^{3}\right) f\left(-q^{11}\right) f\left(-q^{33}\right) \\
& +9 q \sqrt{f(-q) f\left(-q^{3}\right) f\left(-q^{11}\right) f\left(-q^{33}\right)} \\
& \quad \cdot\left\{3 q^{2} \sqrt{\frac{f^{3}\left(-q^{3}\right) f^{3}\left(-q^{33}\right)}{f(-q) f\left(-q^{11}\right)}}+\sqrt{\frac{f^{3}(-q) f^{3}\left(-q^{11}\right)}{f\left(-q^{3}\right) f\left(-q^{33}\right)}}\right\} \\
& =\frac{\left\{f^{12}(-q)+27 q f^{12}\left(-q^{3}\right)\right\}^{\frac{1}{3}}\left\{f^{12}\left(-q^{11}\right)+27 q^{11} f^{12}\left(-q^{33}\right)\right\}^{\frac{1}{3}}}{f(-q) f\left(-q^{3}\right) f\left(-q^{11}\right) f\left(-q^{33}\right)} .
\end{aligned}
$$




\section{Setting}

$$
A=f(-q), \quad B=f\left(-q^{3}\right), \quad C=f\left(-q^{11}\right), \quad \text { and } \quad D=f\left(-q^{33}\right),
$$

and multiplying both sides of (7.30) by $A B C D$, we find that it suffices to prove that

$$
\begin{gathered}
9 q^{4} B^{4} D^{4}+A^{4} C^{4}+18 q^{2} A^{2} B^{2} C^{2} D^{2}+27 q^{3} A C B^{3} D^{3}+9 q A^{3} C^{3} B D \\
=\left\{A^{12}+27 q B^{12}\right\}^{\frac{1}{3}}\left\{C^{12}+27 q^{11} D^{12}\right\}^{\frac{1}{3}} .
\end{gathered}
$$

We next cube both sides of (7.31), simplify, and divide both sides of the resulting equality by $27(q A B C D)^{6}$. After considerable algebra, we deduce that

$$
\begin{aligned}
& \left(\frac{A C}{q B D}\right)^{5}+\left(\frac{3 q B D}{A C}\right)^{5}+\left(3^{2}+2\right)\left\{\left(\frac{A C}{q B D}\right)^{4}+\left(\frac{3 q B D}{A C}\right)^{4}\right\} \\
& +\left(3^{3}+4 \cdot 3^{2}+3\right)\left\{\left(\frac{A C}{q B D}\right)^{3}+\left(\frac{3 q B D}{A C}\right)^{3}\right\} \\
& +\left(2 \cdot 3^{4}+2 \cdot 3^{3}+4 \cdot 3^{2}+1\right)\left\{\left(\frac{A C}{q B D}\right)^{2}+\left(\frac{3 q B D}{A C}\right)^{2}\right\} \\
& +\left(3^{5}+4 \cdot 3^{4}+4 \cdot 3^{3}+2 \cdot 3^{2}\right)\left\{\frac{A C}{q B D}+\frac{3 q B D}{A C}\right\} \\
& +\left(4 \cdot 3^{5}+2 \cdot 3^{4}+8 \cdot 3^{3}+4 \cdot 3^{2}\right)=q^{5}\left(\frac{A D}{B C}\right)^{6}+\frac{1}{q^{5}}\left(\frac{B C}{A D}\right)^{6} .
\end{aligned}
$$

Now set

$$
P=\frac{A}{q^{\frac{1}{12}} B}=\frac{\eta(z)}{\eta(3 z)} \quad \text { and } \quad Q=\frac{C}{q^{\frac{11}{12}} D}=\frac{\eta(11 z)}{\eta(33 z)},
$$

where $\eta(z)$ denotes the Dedekind eta-function, $q=\exp (2 \pi i z)$, and $\operatorname{Im}(z)>$ 0 . Then (7.32) is equivalent to the identity

$$
\begin{aligned}
& (P Q)^{5}+\left(\frac{3}{P Q}\right)^{5}+11\left\{(P Q)^{4}+\left(\frac{3}{P Q}\right)^{4}\right\} \\
& +66\left\{(P Q)^{3}+\left(\frac{3}{P Q}\right)^{3}\right\}+253\left\{(P Q)^{2}+\left(\frac{3}{P Q}\right)^{2}\right\} \\
& +693\left\{P Q+\frac{3}{P Q}\right\}+1386=\left(\frac{P}{Q}\right)^{6}+\left(\frac{Q}{P}\right)^{6} .
\end{aligned}
$$

The beautiful eta-function identity (7.33) has the same shape as many etafunction identities found in Ramanujan's notebooks, but is apparently not in Ramanujan's work. In contrast to our proofs of most of these identities, we shall invoke the theory of modular forms to prove (7.33). All of the necessary theory is found in [10] or [8, Chapter 25].

Let $\Gamma(1)$ denote the full modular group, and let $M(\Gamma, r, v)$ denote the space of modular forms of weight $r$ and multiplier system $v$ on $\Gamma$, where $\Gamma$ is a subgroup of finite index in $\Gamma(1)$. As usual, let

$$
\Gamma_{0}(N)=\left\{\left(\begin{array}{ll}
a & b \\
c & d
\end{array}\right) \in \Gamma(1): c \equiv 0(\bmod N)\right\} .
$$


Then by [10, Lemma 1], $P Q \in M\left(\Gamma_{0}(33), 0,1\right)$, and by [10, Lemma 2], $(P / Q)^{6} \in M\left(\Gamma_{0}(33), 0,1\right)$. Let ord $(g ; z)$ denote the invariant order of a modular form $g$ at $z$. Let $r / s,(r, s)=1$, denote a cusp for the group $\Gamma$. Then, for any pair of positive integers $m, n$,

$$
\operatorname{ord}\left(\eta(m n z) ; \frac{r}{s}\right)=\frac{(m n, s)^{2}}{24 m n}
$$

where $\eta$ denotes the Dedekind eta-function.

A complete set of cusps for $\Gamma_{0}(33)$ is $\left\{0, \frac{1}{3}, \frac{1}{11}, \infty\right\}$. Using (7.34), we calculate the orders of $P Q$ and $P / Q$ at each finite cusp. The following table summarizes these calculations:

$\begin{array}{ccc}\text { function } g & \text { cusp } \zeta & \operatorname{ord}(g ; \zeta) \\ P Q & 0 & \frac{1}{33} \\ & \frac{1}{3} & -\frac{1}{11} \\ & \frac{1}{11} & \frac{1}{3} \\ P / Q & 0 & \frac{5}{198} \\ & \frac{1}{3} & -\frac{5}{66} \\ & \frac{1}{11} & -\frac{5}{18}\end{array}$

Let $L$ and $R$ denote the left and right sides, respectively, of (7.33). Using the valence formula (Rankin [38, p. 98, Theorem 4.1.4]) and the table above, we find that

$$
\begin{aligned}
0 & \geq \operatorname{ord}(L ; \infty)+\operatorname{ord}(L ; 0)+\operatorname{ord}\left(L ; \frac{1}{3}\right)+\operatorname{ord}\left(L ; \frac{1}{11}\right) \\
& =\operatorname{ord}(L ; \infty)-\frac{5}{33}-\frac{5}{11}-\frac{5}{3} \\
& =\operatorname{ord}(L ; \infty)-\frac{25}{11}
\end{aligned}
$$

and

$$
0 \geq \operatorname{ord}(R ; \infty)-\frac{5}{33}-\frac{5}{11}-\frac{5}{3}=\operatorname{ord}(R ; \infty)-\frac{25}{11}
$$

By (7.35) and (7.36), if we can show that

$$
L-R=O\left(q^{3}\right),
$$

as $q$ tends to 0 , then we shall have completed the proof of (7.33). Using Mathematica, we find that

$L=q^{-5}+6 q^{-4}+27 q^{-3}+92 q^{-2}+279 q^{-1}+756+1913 q+4536 q^{2}+O\left(q^{3}\right)=R$.

Thus, the proof of (7.37), and hence also of (7.28), is complete.

At the bottom of page 259 [35], Ramanujan records three modular equations of composite degrees. Unfortunately, we have been unable to prove them by methods familiar to Ramanujan and so have resorted to the theory of modular forms for our proofs. It would be of considerable interest if more instructive proofs could be found. Because the proofs are similar, we give the three together. 
Theorem 7.9 (p. 259). If $\beta, \gamma$, and $\delta$ are of degrees 2,4 , and 8 , respectively, and if $m_{1}$ and $m_{2}$ are the multipliers associated with the pairs $\alpha, \beta$ and $\gamma, \delta$, respectively, then

$$
\frac{1-(\alpha \delta)^{\frac{1}{3}}-\{(1-\alpha)(1-\delta)\}^{\frac{1}{3}}}{3\{\beta \gamma(1-\beta)(1-\gamma)\}^{\frac{1}{6}}}=\frac{m_{2}}{m_{1}} .
$$

Theorem 7.10 (p. 259). If $\beta, \gamma$, and $\delta$ have degrees 2,7 , and 14 or 4,5 , and 20 , respectively, and if $m_{1}$ and $m_{2}$ are as in the previous theorem, then

$$
\frac{1+2\left((\alpha \delta)^{\frac{1}{3}}+\{(1-\alpha)(1-\delta)\}^{\frac{1}{3}}\right)}{1+2\left((\beta \gamma)^{\frac{1}{3}}+\{(1-\beta)(1-\gamma)\}^{\frac{1}{3}}\right)}=\frac{m_{2}}{m_{1}} .
$$

Proofs of Theorems 7.9 and 7.10. Transcribing (7.38) and (7.39) via Corollary 3.2 , we see that it suffices to prove that

$$
\begin{gathered}
a(q) a\left(q^{8}\right)-c(q) c\left(q^{8}\right)-b(q) b\left(q^{8}\right)=3 \sqrt{c\left(q^{2}\right) c\left(q^{4}\right) b\left(q^{2}\right) b\left(q^{4}\right)} \\
a(q) a\left(q^{14}\right)+2 c(q) c\left(q^{14}\right)+2 b(q) b\left(q^{14}\right) \\
=a\left(q^{2}\right) a\left(q^{7}\right)+2 c\left(q^{2}\right) c\left(q^{7}\right)+2 b\left(q^{2}\right) b\left(q^{7}\right)
\end{gathered}
$$

and

$$
\begin{aligned}
& a(q) a\left(q^{20}\right)+2 c(q) c\left(q^{20}\right)+2 b(q) b\left(q^{20}\right) \\
& \quad=a\left(q^{4}\right) a\left(q^{5}\right)+2 c\left(q^{4}\right) c\left(q^{5}\right)+2 b\left(q^{4}\right) b\left(q^{5}\right) .
\end{aligned}
$$

Next, employing (5.4), (5.5), and (7.12), we translate (7.40)-(7.42) into the equivalent eta-function identities,

$$
\begin{aligned}
& \frac{\left\{f^{12}(-q)+27 q f^{12}\left(-q^{3}\right)\right\}^{\frac{1}{3}}\left\{f^{12}\left(-q^{8}\right)+27 q^{8} f^{12}\left(-q^{24}\right)\right\}^{\frac{1}{3}}}{f(-q) f\left(-q^{3}\right) f\left(-q^{8}\right) f\left(-q^{24}\right)} \\
& \quad-9 q^{3} \frac{f^{3}\left(-q^{3}\right) f^{3}\left(-q^{24}\right)}{f(-q) f\left(-q^{8}\right)}-\frac{f^{3}(-q) f^{3}\left(-q^{8}\right)}{f\left(-q^{3}\right) f\left(-q^{24}\right)} \\
& =9 q f\left(-q^{2}\right) f\left(-q^{4}\right) f\left(-q^{6}\right) f\left(-q^{12}\right), \\
& \frac{\left\{f^{12}(-q)+27 q f^{12}\left(-q^{3}\right)\right\}^{\frac{1}{3}}\left\{f^{12}\left(-q^{14}\right)+27 q^{14} f^{12}\left(-q^{42}\right)\right\}^{\frac{1}{3}}}{f(-q) f\left(-q^{3}\right) f\left(-q^{14}\right) f\left(-q^{42}\right)} \\
& \quad+18 q^{5} \frac{f^{3}\left(-q^{3}\right) f^{3}\left(-q^{42}\right)}{f(-q) f\left(-q^{14}\right)}+2 \frac{f^{3}(-q) f^{3}\left(-q^{14}\right)}{f\left(-q^{3}\right) f\left(-q^{42}\right)} \\
& =\frac{\left\{f^{12}\left(-q^{2}\right)+27 q^{2} f^{12}\left(-q^{6}\right)\right\}^{\frac{1}{3}}\left\{f^{12}\left(-q^{7}\right)+27 q^{7} f^{12}\left(-q^{21}\right)\right\}^{\frac{1}{3}}}{f\left(-q^{2}\right) f\left(-q^{6}\right) f\left(-q^{7}\right) f\left(-q^{21}\right)} \\
& \quad+18 q^{3} \frac{f^{3}\left(-q^{6}\right) f^{3}\left(-q^{21}\right)}{f\left(-q^{2}\right) f\left(-q^{7}\right)}+2 \frac{f^{3}\left(-q^{2}\right) f^{3}\left(-q^{7}\right)}{f\left(-q^{6}\right) f\left(-q^{21}\right)},
\end{aligned}
$$


and

$$
\begin{aligned}
& \frac{\left\{f^{12}(-q)+27 q f^{12}\left(-q^{3}\right)\right\}^{\frac{1}{3}}\left\{f^{12}\left(-q^{20}\right)+27 q^{20} f^{12}\left(-q^{60}\right)\right\}^{\frac{1}{3}}}{f(-q) f\left(-q^{3}\right) f\left(-q^{20}\right) f\left(-q^{60}\right)} \\
& \quad+18 q^{7} \frac{f^{3}\left(-q^{3}\right) f^{3}\left(-q^{60}\right)}{f(-q) f\left(-q^{20}\right)}+2 \frac{f^{3}(-q) f^{3}\left(-q^{20}\right)}{f\left(-q^{3}\right) f\left(-q^{60}\right)} \\
& =\frac{\left\{f^{12}\left(-q^{4}\right)+27 q^{4} f^{12}\left(-q^{12}\right)\right\}^{\frac{1}{3}}\left\{f^{12}\left(-q^{5}\right)+27 q^{5} f^{12}\left(-q^{15}\right)\right\}^{\frac{1}{3}}}{f\left(-q^{4}\right) f\left(-q^{12}\right) f\left(-q^{5}\right) f\left(-q^{15}\right)} \\
& +18 q^{3} \frac{f^{3}\left(-q^{12}\right) f^{3}\left(-q^{15}\right)}{f\left(-q^{4}\right) f\left(-q^{5}\right)}+2 \frac{f^{3}\left(-q^{4}\right) f^{3}\left(-q^{5}\right)}{f\left(-q^{12}\right) f\left(-q^{15}\right)} .
\end{aligned}
$$

Recall that if $q=\exp (2 \pi i z)$, where $\operatorname{Im}(z)>0$, then $\eta(z)=q^{\frac{1}{24}} f(-q)$ is a modular form on $\Gamma(1)$ of weight $\frac{1}{2}$. If $\left(\begin{array}{ll}a & b \\ c & d\end{array}\right) \in \Gamma(1)$ and $d$ is odd, the multiplier system $v_{\eta}\left(\begin{array}{ll}a & b \\ c & d\end{array}\right)$ is given by [32, p. 51]

$$
v_{\eta}\left(\begin{array}{ll}
a & b \\
c & d
\end{array}\right)= \pm\left(\frac{c}{|d|}\right) e^{2 \pi i\left\{a c\left(1-d^{2}\right)+d(b-c)+3(d-1)\right\} / 24}
$$

where $\left(\frac{c}{|d|}\right)$ denotes the Legendre symbol, the plus sign is taken if $c \geq 0$ or $d \geq 0$, and the minus sign is chosen if $c<0$ and $d<0$. Using (7.46), we find that each of the four expressions in (7.43) and each of the six expressions in both (7.44) and (7.45) has a multiplier system identically equal to 1 , provided, of course, that $c$ is divisible by 24,42 , and 60 , respectively. Hence, both sides of (7.43)-(7.45) belong to $M\left(\Gamma_{0}(n), 2,1\right)$, where $n=24,42$, and 60 , respectively.

If $\sigma_{\infty}$ denotes the number of inequivalent cusps of a fundamental region for $\Gamma_{0}(n)$, then [39, p. 102]

$$
\sigma_{\infty}=\sum_{d \mid n} \phi((d, n / d))
$$

where $\phi$ denotes Euler's $\phi$-function and $(a, b)$ denotes the greatest common divisor of $a$ and $b$. Thus, for $n=24,42$, and 60, there are 8, 8, and 12 cusps, respectively. Using a procedure found in Schoeneberg's book [39, pp. 86-87], we find that $\left\{0, \frac{1}{2}, \frac{1}{3}, \frac{1}{4}, \frac{1}{6}, \frac{1}{8}, \frac{1}{12}, \infty\right\} ;\left\{0, \frac{1}{2}, \frac{1}{3}, \frac{1}{6}, \frac{1}{7}, \frac{1}{14}, \frac{1}{21}, \infty\right\} ;$ and $\left\{0, \frac{1}{2}, \frac{1}{3}, \frac{1}{4}, \frac{1}{5}, \frac{1}{6}, \frac{1}{10}, \frac{1}{12}, \frac{1}{15}, \frac{1}{20}, \frac{1}{30}, \infty\right\}$ constitute complete sets of inequivalent cusps for $\Gamma_{0}(24), \Gamma_{0}(42)$, and $\Gamma_{0}(60)$, respectively. Employing (7.34), we calculate the order of each expression in $(7.43)-(7.45)$ at each finite cusp. In each instance, we find that each order is nonnegative.

Let $F_{24}, F_{42}$, and $F_{60}$ denote the differences of the left and right sides of (7.43)-(7.45), respectively. Since the order of $F_{24}, F_{42}$, and $F_{60}$ at each point of a fundamental set is nonnegative, we deduce from the valence formula that

$$
r \rho_{\Gamma_{0}(n)} \geq \operatorname{ord}\left(F_{n} ; \infty\right), \quad n=24,42,60,
$$

provided that $F_{n}$ is not constant, where $r$ is the weight of $F_{n}$ and

$$
\rho_{\Gamma_{0}(n)}:=\frac{1}{12}\left[\Gamma(1): \Gamma_{0}(n)\right]
$$


Now (Schoeneberg [39, p. 79]),

$$
\left[\Gamma(1): \Gamma_{0}(n)\right]=n \prod_{p \mid n}\left(1+\frac{1}{p}\right),
$$

where the product is over all primes $p$ dividing $n$. Thus, by (7.48) and (7.49), $\rho_{\Gamma_{0}(24)}=4, \rho_{\Gamma_{0}(42)}=8$, and $\rho_{\Gamma_{0}(60)}=12$. Since $r=2$ in each case, by (7.47), (7.50) $\quad \operatorname{ord}\left(F_{24} ; \infty\right) \leq 8, \quad \operatorname{ord}\left(F_{42} ; \infty\right) \leq 16, \quad \operatorname{ord}\left(F_{60} ; \infty\right) \leq 24$, unless $F_{24}, F_{42}$, or $F_{60}$, respectively, is constant.

Using the pentagonal number theorem, (1.14), i.e.,

$$
f(-q)=\sum_{n=-\infty}^{\infty}(-1)^{n} q^{n(3 n-1) / 2}=1-q-q^{2}+q^{5}+q^{7}-q^{12}-q^{15}+q^{22}+\cdots
$$

and Mathematica, we expanded the left and right sides of (7.43)-(7.45) about the cusp $\infty(q=0)$. We found that the left and right sides of (7.43)-(7.45) are equal to, respectively,

$$
\begin{aligned}
9 q-9 q^{3}-18 q^{5}+O\left(q^{9}\right) \\
3+18 q^{3}+18 q^{5}+18 q^{6}+36 q^{7}+54 q^{9}+18 q^{10}+36 q^{11} \\
+18 q^{12}+18 q^{13}+36 q^{14}+90 q^{15}+O\left(q^{17}\right)
\end{aligned}
$$

and

$$
\begin{aligned}
3+18 q^{3} & +18 q^{7}+18 q^{8}+54 q^{9}+36 q^{11}+36 q^{12}+36 q^{13}+18 q^{15} \\
& +36 q^{16}+36 q^{17}+36 q^{19}+126 q^{21}+54 q^{23}+90 q^{24}+O\left(q^{25}\right) .
\end{aligned}
$$

Thus, $F_{24}=O\left(q^{9}\right), F_{42}=O\left(q^{17}\right)$, and $F_{60}=O\left(q^{25}\right)$, which contradicts $(7.50)$ unless $F_{24}, F_{42}$, and $F_{60}$ are each constant. These constants are obviously equal to 0 , and hence (7.43)-(7.45) are established. This completes the proofs of Theorems 7.9 and 7.10.

From (7.51) and (7.52), we are led to several interesting conjectures about the coefficients in the expansions of the left and right sides of (7.44) and (7.45). We will not further pursue this here.

8. The inversion of an analogue of $K(k)$ in Signature 3

Theorem 8.1 (p. 257). Let $q=q_{3}$ be defined by (1.7), and let $z$ be defined by (1.6) with $r=3$. For $0 \leq \phi \leq \pi / 2$, define $\theta=\theta(\phi)$ by

$$
\theta z=\int_{0}^{\phi}{ }_{2} F_{1}\left(\frac{1}{3}, \frac{2}{3} ; \frac{1}{2} ; x \sin ^{2} t\right) d t .
$$

Then, for $0 \leq \theta \leq \pi / 2$,

$$
\phi=\theta+3 \sum_{n=1}^{\infty} \frac{\sin (2 n \theta)}{n(1+2 \cosh (n y))}=\theta+3 \sum_{n=1}^{\infty} \frac{\sin (2 n \theta) q^{n}}{n\left(1+q^{n}+q^{2 n}\right)}=: \Phi(\theta),
$$

where $q:=e^{-y}$,

Recall from Entry 35(iii) of Chapter 11 [4, p. 99] that

$$
{ }_{2} F_{1}\left(\frac{1}{2}+n, \frac{1}{2}-n ; \frac{1}{2} ; x^{2}\right)=\left(1-x^{2}\right)^{-\frac{1}{2}} \cos \left(2 n \sin ^{-1} x\right),
$$


where $n$ is arbitrary. With $n=\frac{1}{6}$ in (8.3), we see that the integral in (8.1) is an analogue of the incomplete integral of the first kind, which arises from the case $n=0$ in $(8.3)$. Since ${ }_{2} F_{1}\left(\frac{1}{3}, \frac{2}{3} ; \frac{1}{2} ; x \sin ^{2} t\right)$ is a nonnegative, monotonically increasing function on $[0, \pi / 2]$, there exists a unique inverse function $\phi=\phi(\theta)$. Thus, (8.2) gives the "Fourier series" of this inverse function and is analogous to familiar Fourier series for the Jacobian elliptic functions [42, pp. 511-512]. The function $\phi$ may therefore be considered a cubic analogue of the Jacobian functions. Theorem 8.1 is also remindful of some new inversion formulas in the classical setting which are found on pages 283, 285, and 286 in Ramanujan's second notebook [35] and which have been proved by Berndt and Bhargava [9].

When $\phi=0=\theta,(8.2)$ is trivial. When $\phi=\pi / 2$,

$$
\begin{aligned}
\int_{0}^{\phi}{ }_{2} F_{1}\left(\frac{1}{3}, \frac{2}{3} ; \frac{1}{2} ; x \sin ^{2} t\right) d t & =\sum_{n=0}^{\infty} \frac{\left(\frac{1}{3}\right)_{n}\left(\frac{2}{3}\right)_{n}}{\left(\frac{1}{2}\right)_{n} n !} x^{n} \int_{0}^{\pi / 2} \sin ^{2 n} t d t \\
& =\sum_{n=0}^{\infty} \frac{\left(\frac{1}{3}\right)_{n}\left(\frac{2}{3}\right)_{n}}{\left(\frac{1}{2}\right)_{n} n !} x^{n} \frac{\left(\frac{1}{2}\right)_{n}}{n !} \frac{\pi}{2} \\
& =\frac{\pi}{2}{ }_{2} F_{1}\left(\frac{1}{3}, \frac{2}{3} ; 1 ; x\right) .
\end{aligned}
$$

Thus, $\theta=\pi / 2$, which is implicit in our statement of Theorem 8.1.

We now give an outline of the proof of Theorem 8.1. Returning to (8.3), we observe that

$$
S(x):={ }_{2} F_{1}\left(\frac{1}{3}, \frac{2}{3} ; \frac{1}{2} ; x^{2}\right)=\left(1-x^{2}\right)^{-\frac{1}{2}} \cos \left(\frac{1}{3} \sin ^{-1} x\right), \quad|x|<1,
$$

is that unique, real valued function on $(-1,1)$ satisfying the properties

$$
S(x) \text { is continuous on }(-1,1) \text {, }
$$

and

$$
S(0)=1
$$

Properties (8.5) and (8.6) are obvious, and (8.7) follows from the elementary identity $4 \cos ^{3} \theta=3 \cos \theta+\cos (3 \theta)$. To see that $S(x)$ is unique, set $y=S(x)$ in (8.7) and solve for $x^{2}$. Thus,

$$
x^{2}=\frac{(y-1)(1+2 y)^{2}}{4 y^{3}} .
$$

Since $S(x)$ is real valued, either $y<0$ or $y \geq 1$. Since $S(0)=1$ and $S$ is continuous, we conclude that $y \geq 1$. Hence

$$
x= \pm g(y)
$$

where

$$
g(y):=\frac{\sqrt{1-1 / y}(2 y+1)}{2 y} .
$$


Now, $g(y)$ is monotonically increasing on $[1, \infty)$. Thus, $g^{-1}(x)$ exists, and if $0 \leq x<1, y=g^{-1}(x)$, while if $-1<x<0$, we have $y=g^{-1}(-x)$. Thus, $S(x)$ is uniquely determined.

We fix $q, 0<q<1$. Set $x=c^{3}(q) / a^{3}(q)$, so by (2.5), $0<x<1$. Then, by Lemma $2.6, Z:={ }_{2} F_{1}\left(\frac{1}{3}, \frac{2}{3} ; 1 ; x\right)=a(q)$. (We use the notation $Z$ instead of $z=z(3)$, because in this section $z$ will denote a complex variable.) With $\Phi(\theta)$ defined in (8.2), we shall prove that

$$
\frac{d \Phi(\theta)}{d \theta}>0, \quad 0<\theta<\pi / 2
$$

and

$$
4 x \sin ^{2}(\Phi(\theta))=4-\frac{1}{Z^{3}}\left(\frac{d \Phi}{d \theta}\right)^{3}-\frac{3}{Z^{2}}\left(\frac{d \Phi}{d \theta}\right)^{2} .
$$

By $(8.8)$, we may define $\Theta:=\Phi^{-1}:[0, \pi / 2] \rightarrow[0, \pi / 2]$. Setting $S:=$ $Z d \Theta / d \phi$, we see from (8.9) that

$$
4 S^{3}\left(1-x \sin ^{2} \phi\right)-3 S-1=0 .
$$

Hence, (8.7) holds with $x^{2}$ replaced by $x \sin ^{2} \phi$. Now, from (8.2),

$$
\Phi^{\prime}(0)=1+6 \sum_{n=1}^{\infty} \frac{q^{n}}{1+q^{n}+q^{2 n}}=Z,
$$

by Theorem 2.12. Thus,

$$
S(0)=Z / \Phi^{\prime}(0)=1 \text {. }
$$

Hence, by $(8.4)-(8.7)$, we conclude that

$$
Z \frac{d \Theta}{d \phi}={ }_{2} F_{1}\left(\frac{1}{3}, \frac{2}{3} ; \frac{1}{2} ; x \sin ^{2} \phi\right), \quad 0<\phi<\pi / 2,
$$

and so

$$
Z \Theta(\phi)=\int_{0}^{\phi}{ }_{2} F_{1}\left(\frac{1}{3}, \frac{2}{3} ; \frac{1}{2} ; x \sin ^{2} t\right) d t, \quad 0 \leq \phi \leq \pi / 2,
$$

since $\Theta(0)=0$. It follows that $\Theta(\phi)=\theta(\phi)$ and that $\phi=\Phi(\theta)$. Hence, the desired result (8.2) holds.

Proof of Theorem 8.1. For $|q|<|z|<1 /|q|$, define

$$
v(z, q):=1+3 \sum_{n=1}^{\infty} \frac{\left(z^{n}+z^{-n}\right) q^{n}}{1+q^{n}+q^{2 n}} .
$$

We note, by Theorem 2.12 , that $v(1, q)=a(q)$, and, by $(8.2)$, that

$$
V(\theta):=v\left(e^{2 i \theta}, q\right)=\frac{d \Phi}{d \theta} .
$$

By expanding $1 /\left(1-q^{3 n}\right)$ in a geometric series and inverting the order of summation, we find that

$$
\begin{aligned}
v(z, q)=1+3 \sum_{n=0}^{\infty}\left\{\frac{z q^{3 n+1}}{1-z q^{3 n+1}}-\frac{z q^{3 n+2}}{1-z q^{3 n+2}}\right. & \\
& \left.\quad+\frac{z^{-1} q^{3 n+1}}{1-z^{-1} q^{3 n+1}}-\frac{z^{-1} q^{3 n+2}}{1-z^{-1} q^{3 n+2}}\right\} .
\end{aligned}
$$


As a function of $z, v(z, q)$ can be analytically continued to $C \backslash\{0\}$, where the analytic continuation $v(z, q)$ is analytic except for simple poles at $z=q^{m}$, where $m$ is an integer such that $m \not \equiv 0(\bmod 3)$. Using $(8.12)$, we find, by a straightforward calculation, that

$$
v\left(z q^{3}, q\right)=v(z, q) \text {. }
$$

Hirschhorn, Garvan, and J. Borwein [31] have studied generalizations of $a(q), b(q)$, and $c(q)$ in two variables. In particular, they defined

$$
b(z, q):=\sum_{m, n=-\infty}^{\infty} \omega^{m-n} q^{m^{2}+m n+n^{2}} z^{n}
$$

and showed that $[31$, eq. $(1.22)]$

$$
\begin{aligned}
b(z, q) & =(q ; q)_{\infty}\left(q^{3} ; q^{3}\right)_{\infty} \frac{(z q ; q ;)_{\infty}\left(z^{-1} q ; q\right)_{\infty}}{\left(z q^{3} ; q^{3}\right)_{\infty}\left(z^{-1} q^{3} ; q^{3}\right)_{\infty}} \\
& =\prod_{n=1}^{\infty} \frac{\left(1-q^{n}\right)^{3}}{1-q^{3 n}} \prod_{3 \nmid n}^{\infty} \frac{\left(1-z q^{n}\right)\left(1-z^{-1} q^{n}\right)}{\left(1-q^{n}\right)^{2}}
\end{aligned}
$$

and $[31$, eq. $(1.17)]$

$$
b\left(z q^{3}, q\right)=z^{-2} q^{-3} b(z, q) .
$$

We next show that $v(z, q)$ can be written in terms of $b(z, q)$ and $b(-z, q)$.

Lemma 8.2. If

$$
\alpha(q):=\frac{(q ; q)_{\infty}^{2}\left(q^{3} ; q^{3}\right)_{\infty}^{2}}{\left(q^{2} ; q^{2}\right)_{\infty}\left(q^{6} ; q^{6}\right)_{\infty}}
$$

and

$$
\beta(q):=\frac{(q ; q)_{\infty}^{6}\left(q^{6} ; q^{6}\right)_{\infty}}{\left(q^{2} ; q^{2}\right)_{\infty}^{3}\left(q^{3} ; q^{3}\right)_{\infty}^{2}}
$$

then

$$
v(z, q)=\frac{3}{2} \alpha(q) \frac{b(-z, q)}{b(z, q)}-\frac{1}{2} \beta(q) .
$$

The case $z=1$ of (8.16) follows from [31, eq. (1.29)]. To prove Lemma 8.2, we employ the following lemma due to A.O.L. Atkin and P. Swinnerton-Dyer [1].

Lemma 8.3. Let $q, 0<q<1$, be fuxed. Suppose that $f(z)$ is an analytic function of $z$, except for possibly a finite number of poles, in every region, $0<z_{1} \leq|z| \leq z_{2}$. If

$$
f(z q)=A z^{k} f(z)
$$

for some integer $k$ (positive, zero, or negative) and some constant $A$, then either $f(z)$ has $k$ more poles than zeros in the region $|q|<|z| \leq 1$, or $f(z)$ vanishes identically.

Proof of Lemma 8.2. Define

$$
F(z):=b(z, q) v(z, q)-\frac{3}{2} \alpha(q) b(-z, q)+\frac{1}{2} \beta(q) b(z, q) .
$$


Examining (8.16), we see that our goal is to prove that $F(z) \equiv 0$. From $(8.13)$ and (8.15),

$$
F\left(z q^{3}\right)=z^{-2} q^{-3} F(z) .
$$

From our previous identification of the poles of $v(z, q)$ and from the definition (8.14) of $b(z, q)$, we see that the singularities of $F(z)$ are removable. Thus, by Lemma 8.3 , to show that $F(z) \equiv 0$, we need only show that $F(z)=0$ for three distinct values of $z$ in the region $|q|^{3}<|z| \leq 1$. We choose the values $z=-1, \omega, \omega^{2}$, where $\omega=\exp (2 \pi i / 3)$.

For $z=-1$, by $(8.17)$ and $(8.14)$, we want to prove that

$$
v(-1, q)=\frac{3}{2} \alpha(q) \frac{(q ; q)_{\infty}^{4}\left(q^{6} ; q^{6}\right)_{\infty}^{2}}{\left(q^{3} ; q^{3}\right)_{\infty}^{4}\left(q^{2} ; q^{2}\right)_{\infty}^{2}}-\frac{1}{2} \beta(q)=\beta(q)
$$

But this has been proved by N. Fine [24, p. 84, eq. (32.64)].

For the values $z=\omega, \omega^{2}$, we need the evaluations

$$
v(\omega, q)=v\left(\omega^{2}, q\right)=b(q)
$$

The first equality follows from the representation of $v(z, q)$ in (8.12). To prove the second, we first find from (8.10) that

$$
\frac{1}{2} v(1, q)+v(\omega, q)=\frac{3}{2}+9 \sum_{n=1}^{\infty} \frac{q^{3 n}}{1+q^{3 n}+q^{6 n}}=\frac{3}{2} a\left(q^{3}\right)
$$

by Theorem 2.12 . Since $v(1, q)=a(q)$, by Theorem 2.12 , we deduce that

$$
v(\omega, q)=\frac{3}{2} a\left(q^{3}\right)-\frac{1}{2} a(q)
$$

By (2.8), we conclude that $b(q)=v(\omega, q)$ to complete the proof of (8.19).

Now setting $z=\omega$, we see from (8.17) that we are required to prove that

$$
\begin{aligned}
b(q) & =\frac{3}{2} \alpha(q) \frac{b(-\omega, q)}{b(\omega, q)}-\frac{1}{2} \beta(q) \\
& =\frac{3}{2} \frac{(q ; q)_{\infty}^{4}\left(q^{6} ; q^{6}\right)_{\infty}\left(q^{9} ; q^{9}\right)_{\infty}^{2}}{\left(q^{2} ; q^{2}\right)_{\infty}^{2}\left(q^{3} ; q^{3}\right)_{\infty}^{2}\left(q^{18} ; q^{18}\right)_{\infty}}-\frac{1}{2} \beta(q) \\
& =\frac{\varphi^{2}(-q)}{2 \varphi\left(-q^{3}\right)}\left(3 \varphi\left(-q^{9}\right)-\varphi(-q)\right)
\end{aligned}
$$

where we employed (8.14), much simplification, and (5.1). From Entry 1(iii) in Chapter 20 of Ramanujan's second notebook [5, p. 345],

$$
3 \frac{\varphi\left(-q^{9}\right)}{\varphi(-q)}-1=\left(9 \frac{\varphi^{4}\left(-q^{3}\right)}{\varphi^{4}(-q)}-1\right)^{\frac{1}{3}} .
$$


Thus, by (5.9), (1.17), (3.10), and (3.11),

$$
\begin{aligned}
\frac{\varphi^{3}(-q)}{2 \varphi\left(-q^{3}\right)}\left(3 \frac{\varphi\left(-q^{9}\right)}{\varphi(-q)}-1\right) & =\frac{\varphi^{3}(-q)}{2 \varphi\left(-q^{3}\right)}\left(9 \frac{\varphi^{4}\left(-q^{3}\right)}{\varphi^{4}(-q)}-1\right)^{\frac{1}{3}} \\
& =\frac{z_{1}^{\frac{3}{2}}(1-\alpha)^{\frac{3}{4}}}{2 z_{3}^{\frac{1}{2}}(1-\beta)^{\frac{1}{4}}}\left(\frac{9}{m^{2}} \frac{1-\beta}{1-\alpha}-1\right)^{\frac{1}{3}} \\
& =\frac{z_{1}^{\frac{3}{2}}(3-m)^{2}}{8 z_{3}^{\frac{1}{2}} m^{2}}\left(\frac{9}{m^{2}} \frac{m^{2}(m+1)^{2}}{(3-m)^{2}}-1\right)^{\frac{1}{3}} \\
& =\frac{(3-m)^{\frac{4}{3}} \sqrt{z_{1} z_{3}}}{4 m}\left(m^{2}+3 m\right)^{\frac{1}{3}} \\
& =b(q),
\end{aligned}
$$

by (2.11). (See also [12, p. 143, Theorem 4.11(b)].) Thus, (8.20) has been proved. (Note that in (8.21) $\alpha$ and $\beta$ are squares of moduli and are not to be confused with the definitions of $\alpha(q)$ and $\beta(q)$ in Lemma 8.2.)

In conclusion, we have shown that $F(z)=0$ for $z=-1, \omega, \omega^{2}$, and so the proof of Lemma 8.2 is complete.

We shall need some further relations among $a(q), c(q), \alpha(q)$, and $\beta(q)$. First, from (8.21),

$$
b^{3}(q)=\frac{\varphi^{9}(-q)}{8 \varphi^{3}\left(-q^{3}\right)}\left(9 \frac{\varphi^{4}\left(-q^{3}\right)}{\varphi^{4}(-q)}-1\right)=\frac{1}{8} \beta\left(9 \alpha^{2}-\beta^{2}\right) .
$$

Since, from (8.14) and (8.16), with $z=1$,

$$
a(q)=\frac{3 \alpha^{2}}{2 \beta}-\frac{1}{2} \beta
$$

it follows from (2.5) that

$$
c^{3}(q)=\frac{27 \alpha^{4}}{8 \beta^{3}}\left(\alpha^{2}-\beta^{2}\right) .
$$

Recall from (8.11) that $V(\theta)=d \Phi / d \theta$, where $\Phi(\theta)$ is defined by (8.2). Our next task is to derive an infinite product representation for $d V / d \theta$. To do this, we employ Bailey's ${ }_{6} \psi_{6}$ summation [28, p. 239].

Lemma 8.4. Let

and, for $|z|<1$,

$$
\prod\left[\begin{array}{c}
a_{1}, \ldots, a_{m} \\
b_{1}, \ldots, b_{n}
\end{array}\right]:=\frac{\left(a_{1} ; q\right)_{\infty} \cdots\left(a_{m} ; q\right)_{\infty}}{\left(b_{1} ; q\right)_{\infty} \cdots\left(b_{n} ; q\right)_{\infty}}
$$

$$
{ }_{6} \psi_{6}\left[\begin{array}{l}
a_{1}, \ldots, a_{6} \\
b_{1}, \ldots, b_{6}
\end{array} ; ; z\right]:=\sum_{n=-\infty}^{\infty} \frac{\left(a_{1} ; q\right)_{n} \cdots\left(a_{6} ; q\right)_{n}}{\left(b_{1} ; q\right)_{n} \cdots\left(b_{6} ; q\right)_{n}} z^{n} .
$$

Then, for $\left|a^{2} q /(b c d e)\right|<1$,

(8.24)

$$
\begin{aligned}
& { }_{6} \psi_{6}\left[\begin{array}{c}
q \sqrt{a},-q \sqrt{a}, b, c, d, e \\
\sqrt{a},-\sqrt{a}, q a / b, q a / c, q a / d, q a / e
\end{array} ; ; \frac{a^{2} q}{b c d e}\right] \\
& =\prod\left[\begin{array}{c}
a q, a q /(b c), a q /(b d), a q /(b e), a q /(c d), a q /(c e), a q /(d e), q, q / a \\
q / b, q / c, q / d, q / e, a q / b, a q / c, a q / d, a q / e, a^{2} q /(b c d e)
\end{array}\right] .
\end{aligned}
$$


Lemma 8.5. With $z=e^{2 i \theta}$, we have

$$
\begin{aligned}
\frac{d V}{d \theta}= & \frac{d^{2} \Phi}{d \theta^{2}}=-12 \sum_{n=1}^{\infty} \frac{n \sin (2 n \theta) q^{n}}{1+q^{n}+q^{2 n}} \\
= & q(z-1 / z) \frac{\left(z^{2} q^{3} ; q^{3}\right)_{\infty}\left(z^{-2} q^{3} ; q^{3}\right)_{\infty}\left(q ; q^{3}\right)_{\infty}\left(q^{2} ; q^{3}\right)_{\infty}\left(q^{3} ; q^{3}\right)_{\infty}^{4}}{\left(z q ; q^{3}\right)_{\infty}^{2}\left(z^{-1} q ; q^{3}\right)_{\infty}^{2}\left(z q^{2} ; q^{3}\right)_{\infty}^{2}\left(z^{-1} q^{2} ; q^{3}\right)_{\infty}^{2}} \\
= & -12 q \sin (2 \theta) \\
& \cdot \prod_{n=1}^{\infty} \frac{\left(1-2 \cos (4 \theta) q^{3 n}+q^{6 n}\right)\left(1-q^{n}\right)\left(1-q^{3 n}\right)^{3}}{\left(1-2 \cos (2 \theta) q^{3 n-1}+q^{6 n-2}\right)^{2}\left(1-2 \cos (2 \theta) q^{3 n-2}+q^{6 n-4}\right)^{2}}
\end{aligned}
$$

Proof. From (8.10),

$$
\frac{z}{3} \frac{d v}{d z}=\sum_{n=1}^{\infty} \frac{n\left(z^{n}-z^{-n}\right) q^{n}}{1+q^{n}+q^{2 n}} .
$$

By expanding $1 /\left(1-q^{3 n}\right)$ in a geometric series and inverting the order of summation, we find that

$$
\begin{aligned}
\frac{z}{3} \frac{d v}{d z}=\sum_{n=0}^{\infty}\left(\frac{z q^{3 n+1}}{\left(1-z q^{3 n+1}\right)^{2}}\right. & -\frac{z q^{3 n+2}}{\left(1-z q^{3 n+2}\right)^{2}} \\
& \left.-\frac{z^{-1} q^{3 n+1}}{\left(1-z^{-1} q^{3 n+1}\right)^{2}}+\frac{z^{-1} q^{3 n+2}}{\left(1-z^{-1} q^{3 n+2}\right)^{2}}\right) .
\end{aligned}
$$

Replacing $n$ by $-n-1$ in the second and fourth sums and then combining the first and fourth sums and the second and third sums, we find that

$$
\begin{aligned}
& \frac{z}{3} \frac{d v}{d z}=\sum_{n=-\infty}^{\infty}\left(\frac{z q^{3 n+1}}{\left(1-z q^{3 n+1}\right)^{2}}-\frac{z^{-1} q^{3 n+1}}{\left(1-z^{-1} q^{3 n+1}\right)^{2}}\right) \\
& =q(z-1 / z) \sum_{n=-\infty}^{\infty} \frac{\left(1-q^{3 n+1}\right)\left(1+q^{3 n+1}\right)}{\left(1-z q^{3 n+1}\right)^{2}\left(1-z^{-1} q^{3 n+1}\right)^{2}} q^{3 n} \\
& =\frac{q(z-1 / z)\left(1-q^{2}\right)}{(1-z q)^{2}\left(1-z^{-1} q\right)^{2}}{ }_{6} \psi_{6}\left[\begin{array}{c}
q^{4},-q^{4}, z q, z q, z^{-1} q, z^{-1} q \\
q,-q, z q^{4}, z q^{4}, z^{-1} q^{4}, z^{-1} q^{4} ; q^{3} ; q^{3}
\end{array}\right] \\
& =\frac{q(z-1 / z)\left(1-q^{2}\right)}{(1-z q)^{2}\left(1-z^{-1} q\right)^{2}} \\
& \cdot \prod\left[\begin{array}{c}
q^{5}, q^{3} / z^{2}, q^{3}, q^{3}, q^{3}, q^{3}, z^{2} q^{3}, q^{3}, q \\
q^{2} / z, q^{2} / z, q^{2} z, q^{2} z, q^{4} / z, q^{4} / z, q^{4} z, q^{4} z, q^{3}
\end{array}\right] \\
& =q(z-1 / z) \frac{\left(z^{2} q^{3} ; q^{3}\right)_{\infty}\left(z^{-2} q^{3} ; q^{3}\right)_{\infty}\left(q ; q^{3}\right)_{\infty}\left(q^{2} ; q^{3}\right)_{\infty}\left(q^{3} ; q^{3}\right)_{\infty}^{4}}{\left(z q ; q^{3}\right)_{\infty}^{2}\left(z^{-1} q ; q^{3}\right)_{\infty}^{2}\left(z q^{2} ; q^{3}\right)_{\infty}^{2}\left(z^{-1} q^{2} ; q^{3}\right)_{\infty}^{2}},
\end{aligned}
$$

by (8.24). With $z=e^{2 i \theta}$, we thus have shown, by (8.26) and (8.27), that

$$
\begin{aligned}
\frac{d V}{d \theta}= & 2 i z \frac{d v}{d z}=-12 \sum_{n=1}^{\infty} \frac{n \sin (2 n \theta) q^{n}}{1+q^{n}+q^{2 n}} \\
= & -12 q \sin (2 \theta) \\
& \cdot \prod_{n=1}^{\infty} \frac{\left(1-2 \cos (4 \theta) q^{3 n}+q^{6 n}\right)\left(1-q^{n}\right)\left(1-q^{3 n}\right)^{3}}{\left(1-2 \cos (2 \theta) q^{3 n-1}+q^{6 n-2}\right)^{2}\left(1-2 \cos (2 \theta) q^{3 n-2}+q^{6 n-4}\right)^{2}}
\end{aligned}
$$

which is $(8.25)$. 
Next, define

$$
\Psi(\theta)=\frac{1}{4 x}\left(4-\left(\frac{V(\theta)}{Z}\right)^{3}-3\left(\frac{V(\theta)}{Z}\right)^{2}\right)=\frac{1}{4 x Z^{3}}(Z-V)(2 Z+V)^{2}
$$

Thus, (8.9) is equivalent to the identity

$$
\Psi(\theta)=\sin ^{2}(\Phi(\theta)) \text {. }
$$

As a first step in proving (8.29), we establish the following lemma.

Lemma 8.6. With $\Psi$ defined by (8.28),

$$
\left(\frac{d \Psi}{d \theta}\right)^{2}=4 \Psi(1-\Psi)\left(\frac{d \Phi}{d \theta}\right)^{2}
$$

Proof. Differentiating (8.28), we find that

$$
\frac{d \Psi}{d \theta}=-\frac{3 V}{4 x Z^{3}}(V+2 Z) \frac{d V}{d \theta} .
$$

Putting (8.31) in (8.30), recalling that $V(\theta)=d \Phi / d \theta$, and simplifying, we see that it suffices to prove that

$$
9\left(\frac{d V}{d \theta}\right)^{2}=4(Z-V)\left(4 x Z^{3}-(Z-V)(V+2 Z)^{2}\right) .
$$

Employing (8.25) above and using (8.14), we now find that it suffices to prove that

$$
\begin{gathered}
81 q^{2}(z-1 / z)^{2}\left(z^{2} q^{3} ; q^{3}\right)_{\infty}^{2}\left(z^{-2} q^{3} ; q^{3}\right)_{\infty}^{2}(q ; q)_{\infty}^{6}\left(q^{3} ; q^{3}\right)_{\infty}^{10} \\
=b^{4}(z, q)(v-Z)\left(4 x Z^{3}-(Z-v)(v+2 Z)^{2}\right)
\end{gathered}
$$

where $v=v(z, q)$. By a direct calculation, it can be shown that the left side of (8.33) satisfies the functional equation

$$
F\left(z q^{3}, q\right)=z^{-8} q^{-12} F(z, q) .
$$

By (8.13) and (8.15), it is obvious that the right side of (8.33) is also a solution of (8.34). As observed in (8.12), $v(z, q)$ has simple poles at $z=q^{m}$, where $m$ is an integer such that $m \not \equiv 0(\bmod 3)$. From $(8.14)$, we see that $b(z, q)$ has simple zeros at these same points. Thus, the singularities on the right side of (8.33) are removable. In view of Lemma 8.3, it suffices to show that (8.33) is valid for at least nine values of $z$ in the region $\left|q^{3}\right|<|z| \leq 1$.

It is clear that (8.33) holds for $z=1$, since $v(1, q)=a(q)=Z$. In fact, this zero is of order at least 2 on each side, since, by $(8.10), \partial v(z, q) / \partial z$ vanishes at $z=1$.

Next, we show that $(8.33)$ holds for $z=q$. Since $b(z, q)$ has a simple zero at $z=q$, and $v(z, q)$ has a simple pole at $z=q$, we see that we must show that

$$
\begin{aligned}
\lim _{z \rightarrow q}(b(z, q) v(z, q))^{4} & =81 q^{2}(q-1 / q)^{2}\left(q^{5} ; q^{3}\right)_{\infty}^{2}\left(q ; q^{3}\right)_{\infty}^{2}(q ; q)_{\infty}^{6}\left(q^{3} ; q^{3}\right)_{\infty}^{10} \\
& =\left(3(q ; q)_{\infty}^{2}\left(q^{3} ; q^{3}\right)_{\infty}^{2}\right)^{4}
\end{aligned}
$$


But, by (8.12) and (8.14),

$$
\begin{aligned}
\lim _{z \rightarrow q} b(z, q) v(z, q) & =3 \frac{z^{-1} q(q ; q)_{\infty}\left(q^{3} ; q^{3}\right)_{\infty}(z q ; q)_{\infty}\left(z^{-1} q ; q\right)_{\infty}}{\left(1-z^{-1} q\right)\left(z q^{3} ; q^{3}\right)_{\infty}\left(z^{-1} q^{3} ; q^{3}\right)_{\infty}} \\
& =3(q ; q)_{\infty}^{2}\left(q^{3} ; q^{3}\right)_{\infty}^{2}
\end{aligned}
$$

which establishes (8.35). A similar argument shows that (8.33) holds for $z=$ $q^{2}$.

Next, we examine the case $z=\omega$. We shall need to use the equality

$$
a(q)-b(q)=3 c\left(q^{3}\right)
$$

which is a consequence of (2.8) and (2.9). We also shall need the equalities

$$
b(\omega, q)=b\left(q^{3}\right)=b\left(\omega^{2}, q\right),
$$

which are readily verified by means of (8.14).

Recall that $x=c^{3}(q) / a^{3}(q)$ (see the beginning of the paragraph immmediately prior to the proof of Theorem 8.1) and that $Z=a(q)$. Also, by (8.19), $v(\omega, q)=b(q)$. Hence, by $(2.5)$,

$$
\begin{aligned}
4 x Z^{3}-(Z-v(\omega, q))(2 Z+v(\omega, q))^{2} & =4 c^{3}-(a-b)(2 a+b)^{2} \\
& =4\left(a^{3}-b^{3}\right)-(a-b)(2 a+b)^{2} \\
& =3 b^{2}(a-b) .
\end{aligned}
$$

Thus, by (8.36) and (8.37), the proposed equality (8.33) for $z=\omega$ reduces to the equality

$$
-243 q^{2}\left(q^{9} ; q^{9}\right)_{\infty}^{2}(q ; q)_{\infty}^{6}\left(q^{3} ; q^{3}\right)_{\infty}^{8}=-27 b^{4}\left(q^{3}\right) c^{2}\left(q^{3}\right) b^{2}(q)
$$

But this equality is readily verified by using (5.4) and (5.5).

An almost identical argument shows that (8.33) also holds for $z=\omega^{2}$.

From (8.16), (8.22), (8.23), and considerable algebra, we find that

$$
\begin{aligned}
b^{4}(z, q) & (v(z, q)-Z)\left(4 c^{3}(q)-(Z-v(z, q))(2 Z+v(z, q))^{2}\right) \\
= & -\frac{81 \alpha^{2}}{16 \beta^{2}}(\alpha b(z, q)-\beta b(-z, q))(\alpha b(-z, q)-\beta b(z, q)) \\
& \times\left(b(z, q) b(-z, q)\left\{3 \alpha^{2}-\beta^{2}\right\}+\alpha \beta\left\{b^{2}(z, q)+b^{2}(-z, q)\right\}\right) .
\end{aligned}
$$

Hence, both sides of (8.33) are even functions of $z$. Therefore, we have shown that (8.33) holds for 12 values of $z$, namely, \pm 1 (with multiplicities 2) $\pm q, \pm q^{2}, \pm \omega$, and $\pm \omega^{2}$. Thus, (8.33) holds for all values of $z$, and the proof of Lemma 8.6 is complete.

We are now ready to complete the proof of Theorem 8.1.

Proof of Theorem 8.1. From (8.25),

$$
\frac{d V}{d \theta}<0, \quad 0<\theta<\pi / 2 .
$$

From (8.18),

$$
V(\pi / 2)=v(-1, q)=\beta(q)
$$


It follows from (8.38) and (8.39) that

$$
0<\beta(q)=\dot{V}(\pi / 2)<V(\theta)<V(0)=v(1, q)=Z, \quad 0<\theta<\pi / 2 .
$$

Observe that (8.8) follows from (8.11) and (8.40).

Combining (8.31) and (8.38), we find that

$$
0<\Psi(\theta)<\Psi(\pi / 2) \text {. }
$$

We now calculate $\Psi\left(\frac{1}{2} \pi\right)$. By (8.39) and (8.22),

$$
Z-V(\pi / 2)=a(q)-\beta(q)=\frac{3 \alpha^{2}}{2 \beta}-\frac{3}{2} \beta
$$

and

$$
V(\pi / 2)+2 Z=\frac{3 \alpha^{2}}{\beta} .
$$

Thus, by (8.28), (8.42), and (8.43),

$$
\Psi(\pi / 2)=\frac{1}{4 c^{3}(q)}\left(\frac{3 \alpha^{2}}{2 \beta}-\frac{3 \beta}{2}\right) \frac{9 \alpha^{4}}{\beta^{2}}=\frac{27 \alpha^{4}}{8 c^{3}(q) \beta^{3}}\left(\alpha^{2}-\beta^{2}\right)=1,
$$

by (8.23). So, from (8.41),

$$
0<\Psi(\theta)<1, \quad 0<\theta<\pi / 2 .
$$

Now, by (8.38) and (8.31), $d \Psi / d \theta>0$, and again by (8.11) and (8.40), $d \Phi / d \theta>0$. Also noting (8.44), we conclude from (8.30) that

$$
\frac{1}{2 \sqrt{\Psi(\theta)} \sqrt{1-\Psi(\theta)}} \frac{d \Psi}{d \theta}=\frac{d \Phi}{d \theta}, \quad 0<\theta<\pi / 2 .
$$

Since $V(0)=Z$, from $(8.28)$, we see that $\Psi(0)=0$. By definition, $\Phi(0)=0$. Hence, by (8.45),

$$
\begin{aligned}
\Phi(\theta)=\int_{0}^{\theta} \frac{d \Phi}{d t} d t & =\frac{1}{2} \int_{0}^{\theta} \frac{1}{\sqrt{\Psi(t)} \sqrt{1-\Psi(t)}} \frac{d \Psi}{d t} d t \\
& =\frac{1}{2} \int_{0}^{\Psi(\theta)} \frac{d u}{\sqrt{u} \sqrt{1-u}} \\
& =\int_{0}^{\sqrt{\Psi(\theta)}} \frac{d x}{\sqrt{1-x^{2}}}=\arcsin (\sqrt{\Psi(\theta)}),
\end{aligned}
$$

i.e.,

$$
\Psi(\theta)=\sin ^{2}(\Phi(\theta)), \quad 0 \leq \theta \leq \pi / 2 .
$$

This proves (8.29). Thus, (8.8) and (8.9) have been proved, and this finally completes the proof of Theorem 8.1 .

Theorem 8.1 will be used to prove Ramanujan's next result, Theorem 8.7 below.

For each positive integer $r$, define

$$
S_{2 r}:=\sum_{n=1}^{\infty} \frac{n^{2 r} q^{n}}{1+q^{n}+q^{2 n}} .
$$


Ramanujan evaluated $S_{2 r}$ in closed form for $r=1,2,3,4$. Ramanujan's claimed value for $S_{8}$, namely,

$$
S_{8}=\frac{1}{27} x(1+8 x) z^{9}
$$

is actually incorrect. We shall prove a general formula for $S_{2 r}$ from which the values for $S_{2}, S_{4}, S_{6}$, and $S_{8}$ follow. J.M. and P.B. Borwein [16] have evaluated $S_{2}$ but give no details.

Theorem 8.7 (p. 257). For $r \geq 1$,

$$
S_{2 r}=\frac{(-1)^{r}}{6 \cdot 2^{2 r}} s_{2 r}(Z, x, Z),
$$

where the polynomials $s_{2 r}(V, x, Z)$ are defined by (8.49)-(8.52) below. In particular,

$$
\begin{aligned}
& S_{2}=\frac{x}{27} Z^{3}, \\
& S_{4}=\frac{x}{27} Z^{5}, \\
& S_{6}=\frac{x(3+4 x)}{81} Z^{7},
\end{aligned}
$$

and

Proof. Define

$$
S_{8}=\frac{x\left(81+648 x+80 x^{2}\right)}{3^{7}} Z^{9}
$$

$$
q(V, x, Z):=-\frac{4}{9}\left(4 x Z^{3}+(V-Z)(V+2 Z)^{2}\right)(V-Z) .
$$

Then, by (8.32),

$$
q(V, x, Z)=\left(\frac{d V}{d \theta}\right)^{2} .
$$

By a straightforward calculation,

$$
\begin{aligned}
p(V, x, Z) & :=\frac{1}{2} \frac{\partial}{\partial V} q(V, x, Z) \\
& =-\frac{8}{9} V^{3}-\frac{4}{3} V^{2} Z+\frac{4}{3} V Z^{2}-\frac{8}{9} x Z^{3}+\frac{8}{9} Z^{3} \\
& =-\frac{4}{9}\left(2 x Z^{3}+(V-Z)(V+2 Z)(2 V+Z)\right) .
\end{aligned}
$$

From (8.46),

and so, from (8.47),

$$
2 \frac{d V}{d \theta} \frac{d^{2} V}{d \theta^{2}}=\frac{\partial q(V, x, Z)}{\partial V} \frac{d V}{d \theta},
$$

$$
\frac{d^{2} V}{d \theta^{2}}=p(V, x, Z)
$$

For $n \geq 2$, define two sequences of polynomials $s_{n}(V, x, Z)$ and $t_{n}(V, x, Z)$ by

$$
\begin{aligned}
& s_{2}(V, x, Z):=p(V, x, Z), \\
& t_{2}(V, x, Z):=0,
\end{aligned}
$$


and, for $n \geq 3$,

$$
s_{n}(V, x, Z):=t_{n-1}(V, x, Z) p(V, x, Z)+\frac{\partial}{\partial V} \beta_{n-1}(V, x, Z) q(V, x, Z)
$$

and

$$
t_{n}(V, x, Z):=\frac{\partial}{\partial V} s_{n-1}(V, x, Z) .
$$

By using (8.48)-(8.52), we may prove by induction that

$$
\frac{d^{n} V}{d \theta^{n}}=s_{n}(V, x, Z)+t_{n}(V, x, Z) \frac{d V}{d \theta}, \quad n \geq 2 .
$$

Now, from (8.2), (8.11), and the definition of $S_{2 r}$, we readily see by induction on $r$ that

$$
\left.\frac{d^{2 r} V(\theta)}{d \theta^{2 r}}\right|_{\theta=0}=6(-1)^{r} 2^{2 r} S_{2 r}
$$

Since $V^{\prime}(0)=0$ and $V(0)=Z$, we conclude from (8.53) and (8.54) that

$$
S_{2 r}=\frac{(-1)^{r}}{6 \cdot 2^{2 r}} s_{2 r}(Z, x, Z)
$$

A program was devised in MAPLE to calculate $S_{2 r}$. This completes the proof. 102],

We also calculated $S_{2 r}$ by using Theorem 8.1 in another way. By [30, p.

$$
\phi=\sum_{n=1}^{\infty} \frac{1}{n} \operatorname{Res}\left(\Phi^{-1}\right)^{-n} \theta^{n}
$$

where $\operatorname{Res}\left(\Phi^{-1}\right)^{-n}$ denotes the residue of $\left(\Phi^{-1}\right)^{-n}$ at $\phi=0$. By using (8.2), it is easy to prove that

$$
\phi=\theta+3 \sum_{r=0}^{\infty} \frac{(-1)^{r}(2 \theta)^{2 r+1}}{(2 r+1) !} S_{2 r}
$$

Equating coefficients of $\theta^{2 r+1}$ in (8.55) and (8.56), we find that

$$
S_{2 r}=\frac{(-1)^{r}(2 r) ! \operatorname{Res}\left(\Phi^{-1}\right)^{-2 r-1}}{3 \cdot 2^{2 r+1}} \text {. }
$$

We then used Mathematica to expand $\left(Z \Phi^{-1}(\phi)\right)^{-2 r-1}$ in powers of $\phi$ so that the desired residues could be calculated.

After our proof of Theorem 8.1 was completed, L. Shen [40] found another proof based on the classical theory of elliptic functions.

\section{THE THEORY FOR SIGNATURE 4}

The theory for signature 4 is simpler than that for signature 3 , primarily because of Theorem 9.3 below.

Theorem 9.1 (p. 260). For $0<x<1$,

$$
{ }_{2} F_{1}\left(\frac{1}{2}, \frac{1}{2} ; 1 ; \frac{2 x}{1+x}\right)=\sqrt{1+x}{ }_{2} F_{1}\left(\frac{1}{4}, \frac{3}{4} ; 1 ; x^{2}\right) .
$$

Theorem 9.1 is precisely Entry 33(i) in Chapter 11 of Ramanujan's second notebook [35], [4, p. 94]. 
Theorem 9.2. For $0<x<1$,

$$
{ }_{2} F_{1}\left(\frac{1}{2}, \frac{1}{2} ; 1 ; \frac{1-x}{1+x}\right)=\sqrt{\frac{1}{2}(1+x)}{ }_{2} F_{1}\left(\frac{1}{4}, \frac{3}{4} ; 1 ; 1-x^{2}\right) .
$$

Proof. With $x$ complex and $|x|$ sufficiently small, by Entry 33(iv) in Chapter 11 [35], [4, p. 95],

$$
{ }_{2} F_{1}\left(\frac{1}{2}, \frac{1}{2} ; 1 ; x\right)=\frac{1}{\sqrt{1+x}}{ }_{2} F_{1}\left(\frac{1}{4}, \frac{3}{4} ; 1 ; \frac{4 x}{(1+x)^{2}}\right) .
$$

Replacing $x$ by $(1-x) /(1+x)$ in $(9.3)$, we find that, for $|1-x|$ sufficiently small, (9.2) holds. By analytic continuation, (9.2) holds for $0<x<1$.

Theorem 9.3 (p. 260). Let $q=: q(x)$ denote the classical base, and let $q_{4}=$ : $q_{4}(x)$ be defined by (1.8). Then, for $0<x<1$,

$$
q_{4}(x)=q^{2}\left(\frac{2 \sqrt{x}}{1+\sqrt{x}}\right) .
$$

Proof. Dividing (9.2) by (9.1), we find that, for $0<x<1$,

$$
\sqrt{2} \frac{{ }_{2} F_{1}\left(\frac{1}{4}, \frac{3}{4} ; 1 ; 1-x^{2}\right)}{{ }_{2} F_{1}\left(\frac{1}{4}, \frac{3}{4} ; 1 ; x^{2}\right)}=2 \frac{{ }_{2} F_{1}\left(\frac{1}{2}, \frac{1}{2} ; 1 ; 1-\frac{2 x}{1+x}\right)}{{ }_{2} F_{1}\left(\frac{1}{2}, \frac{1}{2} ; 1 ; \frac{2 x}{1+x}\right)} \text {. }
$$

Replacing $x$ by $\sqrt{x}$ and recalling the definitions of $q$ and $q_{4}$, we see that (9.4) follows.

For further consequences of Entries 33(i), (iv) in Chapter 11, see the examples in Section 33 of Chapter 11 [4, pp. 95-96].

Theorem 9.4 (p. 260). For $0<x<1$,

$$
{ }_{2} F_{1}\left(\frac{1}{4}, \frac{3}{4} ; 1 ; 1-\left(\frac{1-x}{1+3 x}\right)^{2}\right)=\sqrt{1+3 x}{ }_{2} F_{1}\left(\frac{1}{4}, \frac{3}{4} ; 1 ; x^{2}\right) .
$$

Proof. Applying (9.1) and then (9.3) with $x$ replaced by $2 x /(1+x)$, we find that, for $x$ complex and $|x|$ sufficiently small,

$$
\begin{aligned}
{ }_{2} F_{1}\left(\frac{1}{4}, \frac{3}{4} ; 1 ; x^{2}\right) & =(1+x)^{-\frac{1}{2}}{ }_{2} F_{1}\left(\frac{1}{2}, \frac{1}{2} ; 1 ; \frac{2 x}{1+x}\right) \\
& =(1+x)^{-\frac{1}{2}}\left(\frac{1+3 x}{1+x}\right)^{-\frac{1}{2}}{ }_{2} F_{1}\left(\frac{1}{4}, \frac{3}{4} ; 1 ; \frac{8 x(1+x)}{(1+3 x)^{2}}\right) \\
& =(1+3 x)^{-\frac{1}{2}}{ }_{2} F_{1}\left(\frac{1}{4}, \frac{3}{4} ; 1 ; 1-\left(\frac{1-x}{1+3 x}\right)^{2}\right) .
\end{aligned}
$$

Thus, (9.5) has been established for $|x|$ sufficiently small, and by analytic continuation, (9.5) is valid for $0<x<1$.

We now describe a process of deducing formulas in the theory of signature 4 from corresponding formulas in the classical setting. Suppose that we have a formula

$$
\Omega\left(x, q^{2}, z\right)=0 .
$$


Let us replace $x$ by $2 \sqrt{x} /(1+\sqrt{x})$. Then by Theorem $9.3, q^{2}$ is replaced by $q_{4}$. By $(9.1)$,

$$
{ }_{2} F_{1}\left(\frac{1}{2}, \frac{1}{2} ; 1 ; \frac{2 \sqrt{x}}{1+\sqrt{x}}\right)=\sqrt{1+\sqrt{x}}{ }_{2} F_{1}\left(\frac{1}{4}, \frac{3}{4} ; 1 ; x\right),
$$

i.e., $z$ is replaced by $\sqrt{1+\sqrt{x}} z(4)$. Hence, from (9.6), we deduce the formula,

$$
\Omega\left(\frac{2 \sqrt{x}}{1+\sqrt{x}}, q_{4}, \sqrt{1+\sqrt{x}} z(4)\right)=0 .
$$

In each proof below, we apply (9.7) to results from Chapter 17 of Ramanujan's second notebook [35], proofs of which are given in [5].

Theorem 9.5 (p. 260). We have

$$
M\left(q_{4}\right)=z^{4}(4)(1+3 x) .
$$

Proof. By Entry 13(i) in Chapter 17 [5, p. 126],

$$
M\left(q^{2}\right)=z^{4}\left(1-x+x^{2}\right) .
$$

Thus, by (9.7),

$$
\begin{aligned}
M\left(q_{4}\right) & =\{\sqrt{1+\sqrt{x}}\}^{4} z^{4}(4)\left(1-\frac{2 \sqrt{x}}{1+\sqrt{x}}+\left(\frac{2 \sqrt{x}}{1+\sqrt{x}}\right)^{2}\right) \\
& =z^{4}(4)\left\{(1+\sqrt{x})^{2}-2 \sqrt{x}(1+\sqrt{x})+4 x\right\} \\
& =z^{4}(4)(1+3 x) .
\end{aligned}
$$

Theorem 9.6 (p. 260). We have

$$
N\left(q_{4}\right)=z^{6}(4)(1-9 x) .
$$

Proof. By Entry 13(ii) in Chapter 17 [5, p. 126],

$$
N\left(q^{2}\right)=z^{6}(1+x)\left(1-\frac{1}{2} x\right)(1-2 x) .
$$

Using (9.7), we deduce that

$$
\begin{aligned}
N\left(q_{4}\right) & =(1+\sqrt{x})^{3} z^{6}(4)\left(1+\frac{2 \sqrt{x}}{1+\sqrt{x}}\right)\left(1-\frac{\sqrt{x}}{1+\sqrt{x}}\right)\left(1-\frac{4 \sqrt{x}}{1+\sqrt{x}}\right) \\
& =z^{6}(4)(1+3 \sqrt{x})(1-3 \sqrt{x}) \\
& =z^{6}(4)(1-9 x) .
\end{aligned}
$$

Theorem 9.7 (p. 260). We have

$$
M\left(q_{4}^{2}\right)=z^{4}(4)\left(1-\frac{3}{4} x\right) .
$$

Proof. By Entry 13(v) of Chapter 17 [5, p. 127],

$$
M\left(q^{4}\right)=z^{4}\left(1-x+\frac{1}{16} x^{2}\right) .
$$


Hence, by (9.7),

$$
\begin{aligned}
M\left(q_{4}^{2}\right) & =(1+\sqrt{x})^{2} z^{4}(4)\left(1-\frac{2 \sqrt{x}}{1+\sqrt{x}}+\frac{x}{4(1+\sqrt{x})^{2}}\right) \\
& =\frac{1}{4} z^{4}(4)\left\{4(1+\sqrt{x})^{2}-8 \sqrt{x}(1+\sqrt{x})+x\right\} \\
& =\frac{1}{4} z^{4}(4)(4-3 x) .
\end{aligned}
$$

Theorem 9.8 (p. 260). We have

$$
N\left(q_{4}^{2}\right)=z^{6}(4)\left(1-\frac{9}{8} x\right) .
$$

Proof. By Entry 13(vi) in Chapter 17 [5, p. 127],

$$
N\left(q^{4}\right)=z^{6}\left(1-\frac{1}{2} x\right)\left(1-x-\frac{1}{32} x^{2}\right) .
$$

Thus, from (9.7),

$$
\begin{aligned}
N\left(q_{4}^{2}\right) & =(1+\sqrt{x})^{3} z^{6}(4)\left(1-\frac{\sqrt{x}}{1+\sqrt{x}}\right)\left(1-\frac{2 \sqrt{x}}{1+\sqrt{x}}-\frac{x}{8(1+\sqrt{x})^{2}}\right) \\
& =\frac{1}{8} z^{6}(4)\left\{8(1+\sqrt{x})^{2}-16 \sqrt{x}(1+\sqrt{x})-x\right\} \\
& =\frac{1}{8} z^{6}(4)(8-9 x) .
\end{aligned}
$$

It is interesting that the two previous results have simpler formulations in the theory of signature 4 than in the classical theory.

Theorem 9.9 (p. 260). We have

$$
q_{4}^{\frac{1}{24}} f\left(-q_{4}\right)=\sqrt{z(4)} 2^{-\frac{1}{4}} x^{\frac{1}{24}}(1-x)^{\frac{1}{12}} .
$$

Proof. From Entry 12(iii) in Chapter 17 [5, p. 124],

$$
f\left(-q^{2}\right)=\sqrt{z} 2^{-\frac{1}{3}}\{x(1-x) / q\}^{\frac{1}{12}} .
$$

Hence, by (9.7),

$$
\begin{aligned}
q_{4}^{\frac{1}{24}} f\left(-q_{4}\right) & =(1+\sqrt{x})^{\frac{1}{4}} \sqrt{z(4)} 2^{-\frac{1}{3}}\left(\frac{2 \sqrt{x}}{1+\sqrt{x}} \frac{1-\sqrt{x}}{1+\sqrt{x}}\right)^{\frac{1}{12}} \\
& =\sqrt{z(4)} 2^{-\frac{1}{4}} x^{\frac{1}{24}}(1+\sqrt{x})^{\frac{1}{12}}(1-\sqrt{x})^{\frac{1}{12}} \\
& =\sqrt{z(4)} 2^{-\frac{1}{4}} x^{\frac{1}{24}}(1-x)^{\frac{1}{12}} .
\end{aligned}
$$

Theorem 9.10 (p. 260). We have

$$
q_{4}^{\frac{1}{12}} f\left(-q_{4}^{2}\right)=\sqrt{z(4)} 2^{-\frac{1}{2}} x^{\frac{1}{12}}(1-x)^{\frac{1}{24}} .
$$

Proof. By Entry 12(iv) in Chapter 17 [5, p. 124],

$$
q^{\frac{1}{6}} f\left(-q^{4}\right)=\sqrt{z} 4^{-\frac{1}{3}}(1-x)^{\frac{1}{24}} x^{\frac{1}{6}} .
$$


Thus, by (9.7),

$$
\begin{aligned}
q_{4}^{\frac{1}{12}} f\left(-q_{4}^{2}\right) & =(1+\sqrt{x})^{\frac{1}{4}} \sqrt{z(4)} 4^{-\frac{1}{3}}\left(\frac{1-\sqrt{x}}{1+\sqrt{x}}\right)^{\frac{1}{24}}\left(\frac{2 \sqrt{x}}{1+\sqrt{x}}\right)^{\frac{1}{6}} \\
& =\sqrt{z(4)} 2^{-\frac{1}{2}} x^{\frac{1}{12}}(1+\sqrt{x})^{\frac{1}{24}}(1-\sqrt{x})^{\frac{1}{24}} \\
& =\sqrt{z(4)} 2^{-\frac{1}{2}} x^{\frac{1}{12}}(1-x)^{\frac{1}{24}} .
\end{aligned}
$$

Recall the definition of $L(q)$ at the beginning of Section 4. The following intriguing formula does not appear in the second notebook but can be found in the first notebook [35].

Theorem 9.11 (p. 214, first notebook). We have

$$
{ }_{2} F_{1}^{2}\left(\frac{1}{4}, \frac{3}{4} ; 1 ; x\right)=2 L\left(q_{4}^{2}\right)-L\left(q_{4}\right) .
$$

Proof. For brevity, set $q=q_{4}$ and $z=z(4)$. From (4.2) and Theorem 9.9,

$$
\begin{aligned}
L(q) & =q \frac{d}{d q} \log \left(q f^{24}(-q)\right) \\
& =q \frac{d}{d q} \log \left(z^{12} 2^{-6} x(1-x)^{2}\right) \\
& =q \frac{d}{d x} \log \left(z^{12} x(1-x)^{2}\right) \frac{d x}{d q} .
\end{aligned}
$$

By Entry 30 in Chapter 11 of Ramanujan's second notebook [4, p. 87], with $q=q_{4}$,

$$
\frac{d q}{d x}=\frac{q}{x(1-x) z^{2}} .
$$

Using (9.9) in (9.8), we deduce that

$$
\begin{aligned}
L(q) & =\left(\frac{12}{z} \frac{d z}{d x}+\frac{1}{x}-\frac{2}{1-x}\right) x(1-x) z^{2} \\
& =12 x(1-x) z \frac{d z}{d x}+(1-3 x) z^{2} .
\end{aligned}
$$

Repeating the same argument, but with $q$ replaced by $q^{2}$ and with an application of Theorem 9.10 instead of Theorem 9.9, we find that

$$
\begin{aligned}
L\left(q^{2}\right) & =\frac{1}{2} q \frac{d}{d q} \log \left(q^{2} f^{24}\left(-q^{2}\right)\right) \\
& =\frac{1}{2} q \frac{d}{d x} \log \left(z^{12} 2^{-12} x^{2}(1-x)\right) \frac{d x}{d q} \\
& =\frac{1}{2}\left(\frac{12}{z} \frac{d z}{d x}+\frac{2}{x}-\frac{1}{1-x}\right) x(1-x) z^{2} \\
& =6 x(1-x) z \frac{d z}{d x}+\frac{1}{2}(2-3 x) z^{2} .
\end{aligned}
$$

Our theorem now easily follows from (9.10) and (9.11).

We conclude this section with a new transformation formula for ${ }_{2} F_{1}\left(\frac{1}{4}, \frac{3}{4} ; 1 ; x\right)$ found also on page 260 . We need to first establish some ancillary lemmas. 
Lemma 9.12. We have

$$
a(q)+a\left(q^{2}\right)=2 \frac{\psi^{3}(q)}{\psi\left(q^{3}\right)}
$$

and

$$
2 a\left(q^{2}\right)-a(q)=\frac{\varphi^{3}(-q)}{\varphi\left(-q^{3}\right)}
$$

Proof. By Entries 4(iii),(iv) in Chapter 19 of Ramanujan's second notebook [5, pp. 226-227, 229],

$$
\frac{\psi^{3}(q)}{\psi\left(q^{3}\right)}=1+3 \sum_{n=0}^{\infty}\left(\frac{q^{6 n+1}}{1-q^{6 n+1}}-\frac{q^{6 n+5}}{1-q^{6 n+5}}\right)
$$

and

$$
\frac{\varphi^{3}(-q)}{\varphi\left(-q^{3}\right)}=1-6 \sum_{n=0}^{\infty}\left(\frac{q^{3 n+1}}{1+q^{3 n+1}}-\frac{q^{3 n+2}}{1+q^{3 n+2}}\right) .
$$

Using (2.6) to calculate the Lambert series for $\frac{1}{2}\left\{a(q)+a\left(q^{2}\right)\right\}$ and comparing it with the Lambert series above, we deduce (9.12). The proof of (9.13) is similar and follows by substituting $x=q^{3 n+1}, q^{3 n+2}$ in the elementary identity

$$
\frac{2 x^{2}}{1-x^{2}}-\frac{x}{1-x}=-\frac{x}{1+x} \text {. }
$$

Lemma 9.13. We have

$$
\frac{\psi^{6}(q)}{\psi^{2}\left(q^{3}\right)}+18 q \psi^{2}(q) \psi^{2}\left(q^{3}\right)-27 q^{2} \frac{\psi^{6}\left(q^{3}\right)}{\psi^{2}(q)}=\varphi^{4}(q)+16 q \psi^{4}\left(q^{2}\right)
$$

and

$$
\frac{\psi^{6}(q)}{\psi^{2}\left(q^{3}\right)}-6 q \psi^{2}(q) \psi^{2}\left(q^{3}\right)-3 q^{2} \frac{\psi^{6}\left(q^{3}\right)}{\psi^{2}(q)}=\varphi^{4}\left(q^{3}\right)+16 q^{3} \psi^{4}\left(q^{6}\right)
$$

Proof. For brevity, set $a:=a(q)$ and $A:=a\left(q^{2}\right)$. Then, squaring (9.12), we find that

$$
4 \frac{\psi^{6}(q)}{\psi^{2}\left(q^{3}\right)}=(a+A)^{2}
$$

From (5.7), (6.3), and (9.12),

$$
12 q \psi^{2}(q) \psi^{2}\left(q^{3}\right)=a^{2}-A^{2}
$$

and

$$
36 q^{2} \frac{\psi^{6}\left(q^{3}\right)}{\psi^{2}(q)}=(a-A)^{2}
$$

Hence, from (9.16)-(9.18),

$$
\frac{\psi^{6}(q)}{\psi^{2}\left(q^{3}\right)}+18 q \psi^{2}(q) \psi^{2}\left(q^{3}\right)-27 q^{2} \frac{\psi^{6}\left(q^{3}\right)}{\psi^{2}(q)}=a^{2}+2 a A-2 A^{2} .
$$

Next, from (9.16) and (9.18),

$$
48 q \psi^{8}(q)=\left(8 \frac{\psi^{9}(q)}{\psi^{3}\left(q^{3}\right)}\right)\left(6 q \frac{\psi^{3}\left(q^{3}\right)}{\psi(q)}\right)=(a+A)^{3}(a-A)
$$


and

$$
432 q^{3} \psi^{8}\left(q^{3}\right)=\left(216 q^{3} \frac{\psi^{9}\left(q^{3}\right)}{\psi^{3}(q)}\right)\left(2 \frac{\psi^{3}(q)}{\psi\left(q^{3}\right)}\right)=(a-A)^{3}(a+A) .
$$

From (5.6) and (6.5),

$$
3 \frac{\varphi^{3}\left(-q^{3}\right)}{\varphi(-q)}=a+2 A .
$$

Thus, from (9.13) and (9.22),

$$
\varphi^{8}(-q)=\frac{\varphi^{9}(-q)}{\varphi^{3}\left(-q^{3}\right)} \frac{\varphi^{3}\left(-q^{3}\right)}{\varphi(-q)}=\frac{1}{3}(2 A-a)^{3}(a+2 A)
$$

and

$$
\varphi^{8}\left(-q^{3}\right)=\frac{\varphi^{9}\left(-q^{3}\right)}{\varphi^{3}(-q)} \frac{\varphi^{3}(-q)}{\varphi\left(-q^{3}\right)}=\frac{1}{27}(a+2 A)^{3}(2 A-a)
$$

Lastly, we need the elementary identity [5, p. 40, Entry 25(iv)]

$$
\psi\left(q^{4}\right) \varphi\left(q^{2}\right)=\psi^{2}\left(q^{2}\right)
$$

Hence, from (2.1), (9.25), (9.23), and (9.20),

$$
\begin{aligned}
\left\{\varphi^{4}(q)+16 q \psi^{4}\left(q^{2}\right)\right\}^{2} & =\left\{\varphi^{4}(q)-16 q \psi^{4}\left(q^{2}\right)\right\}^{2}+64 q \varphi^{4}(q) \psi^{4}\left(q^{2}\right) \\
& =\varphi^{8}(-q)+64 q \psi^{8}(q) \\
& =\frac{1}{3}(2 A-a)^{3}(a+2 A)+\frac{4}{3}(a+A)^{3}(a-A) \\
& =a^{4}+4 A^{4}+4 a A\left(a^{2}-2 A^{2}\right) \\
& =\left(a^{2}-2 A^{2}\right)^{2}+4 a A\left(a^{2}-2 A^{2}\right)+4 a^{2} A^{2} \\
& =\left(a^{2}+2 a A-2 A^{2}\right)^{2} .
\end{aligned}
$$

Equality (9.14) now follows from (9.19) and (9.26), upon taking the square root of both sides of (9.26) and checking agreement at $q=0$ to ensure that the correct square root is taken.

The proof of (9.15) is similar. Thus, from (9.16)-(9.18),

$$
\frac{\psi^{6}(q)}{\psi^{2}\left(q^{3}\right)}-6 q \psi^{2}(q) \psi^{2}\left(q^{3}\right)-3 q^{2} \frac{\psi^{6}\left(q^{3}\right)}{\psi^{2}(q)}=\frac{1}{3}\left(2 A^{2}+2 a A-a^{2}\right) .
$$

Proceeding as in the proof of (9.14), from (2.1), (9.25), (9.24), and (9.21), we find that

$$
\begin{aligned}
\left\{\varphi^{4}\left(q^{3}\right)+16 q^{3} \psi^{4}\left(q^{6}\right)\right\}^{2} & =\varphi^{8}\left(-q^{3}\right)+64 q^{3} \psi^{8}\left(q^{3}\right) \\
& =\frac{1}{27}(a+2 A)^{3}(2 A-a)+\frac{4}{27}(a-A)^{3}(a+A) \\
& =\frac{1}{9}\left(a^{4}+4 A^{4}+4 a A\left(2 A^{2}-a^{2}\right)\right) \\
& =\frac{1}{9}\left(\left(a^{2}-2 A^{2}\right)^{2}-4 a A\left(a^{2}-2 A^{2}\right)+4 a^{2} A^{2}\right) \\
& =\frac{1}{9}\left(a^{2}-2 A^{2}-2 a A\right)^{2}
\end{aligned}
$$


Equality (9.15) now follows from (9.27) and (9.28).

Lastly, we need the following lemma connecting ${ }_{2} F_{1}\left(\frac{1}{4}, \frac{3}{4} ; 1 ; x\right)$ with thetafunctions.

Lemma 9.14. We have

$$
{ }_{2} F_{1}^{2}\left(\frac{1}{4}, \frac{3}{4} ; 1 ;\left(\frac{8 \sqrt{q} \psi^{2}\left(q^{2}\right) \varphi^{2}(q)}{\varphi^{4}(q)+16 q \psi^{4}\left(q^{2}\right)}\right)^{2}\right)=\varphi^{4}(q)+16 q \psi^{4}\left(q^{2}\right) .
$$

Proof. Let

$$
x=\frac{8 q \psi^{2}\left(q^{4}\right) \varphi^{2}\left(q^{2}\right)}{\varphi^{4}\left(q^{2}\right)+16 q^{2} \psi^{4}\left(q^{4}\right)} .
$$

Then

$$
\frac{2 x}{1+x}=\frac{16 q \psi^{2}\left(q^{4}\right) \varphi^{2}\left(q^{2}\right)}{\left\{\varphi^{2}\left(q^{2}\right)+4 q \psi^{2}\left(q^{4}\right)\right\}^{2}}=16 q \frac{\psi^{4}\left(q^{2}\right)}{\varphi^{4}(q)},
$$

where we have employed (9.25) and the elementary identity

$$
\varphi^{2}\left(q^{2}\right)+4 q \psi^{2}\left(q^{4}\right)=\varphi^{2}(q),
$$

which is achieved by adding Entries $25(\mathrm{v}),(\mathrm{vi})$ in Chapter 16 of Ramanujan's second notebook [35], [5, p. 40]. Hence, from Theorem 9.1, (9.30), (9.31), (2.1), and (5.19), with $-q^{3}$ replaced by $q$,

$$
\begin{aligned}
{ }_{2} F_{1}^{2} & \left(\frac{1}{4}, \frac{3}{4} ; 1 ; x^{2}\right) \\
& =\frac{1}{1+x}{ }_{2} F_{1}^{2}\left(\frac{1}{2}, \frac{1}{2} ; 1 ; \frac{2 x}{1+x}\right) \\
& =\frac{\varphi^{4}\left(q^{2}\right)+16 q^{2} \psi^{4}\left(q^{4}\right)}{\left\{\varphi^{2}\left(q^{2}\right)+4 q \psi^{2}\left(q^{4}\right)\right\}^{2}}{ }_{2} F_{1}^{2}\left(\frac{1}{2}, \frac{1}{2} ; 1 ; 16 q \frac{\psi^{4}\left(q^{2}\right)}{\varphi^{4}(q)}\right) \\
& =\frac{\varphi^{4}\left(q^{2}\right)+16 q^{2} \psi^{4}\left(q^{4}\right)}{\varphi^{4}(q)} \varphi^{4}(q) \\
& =\varphi^{4}\left(q^{2}\right)+16 q^{2} \psi^{4}\left(q^{4}\right) .
\end{aligned}
$$

Replacing $q^{2}$ by $q$, we deduce (9.29).

Lemma 9.14 was first proved by J.M. and P.B. Borwein [12, p. 179, Prop. 5.7(a)], [16, Theorem 2.6(b)].

Theorem 9.15 (p. 260). If

$$
\alpha=\frac{64 p}{\left(3+6 p-p^{2}\right)^{2}} \quad \text { and } \quad \beta=\frac{64 p^{3}}{\left(27-18 p-p^{2}\right)^{2}},
$$

then, for $0 \leq p<1$,

$$
\sqrt{27-18 p-p^{2}}{ }_{2} F_{1}\left(\frac{1}{4}, \frac{3}{4} ; 1 ; \alpha\right)=3 \sqrt{3+6 p-p^{2}}{ }_{2} F_{1}\left(\frac{1}{4}, \frac{3}{4} ; 1 ; \beta\right) \text {. }
$$

Proof. Let

$$
p=9 q \frac{\psi^{4}\left(q^{3}\right)}{\psi^{4}(q)}
$$


Then, by (9.14) and (9.15), respectively,

$$
3+6 p-p^{2}=\frac{\sqrt{p}}{\sqrt{q} \psi^{4}(q)}\left\{\varphi^{4}(q)+16 q \psi^{4}\left(q^{2}\right)\right\}
$$

and

$$
27-18 p-p^{2}=\frac{p^{\frac{3}{2}}}{q^{\frac{3}{2}} \psi^{4}\left(q^{3}\right)}\left\{\varphi^{4}\left(q^{3}\right)+16 q^{3} \psi^{4}\left(q^{6}\right)\right\} .
$$

From (9.32) and (9.35),

$$
\alpha[q]:=\alpha=\frac{64 q \psi^{8}(q)}{\left\{\varphi^{4}(q)+16 q \psi^{4}\left(q^{2}\right)\right\}^{2}}=\left(\frac{8 \sqrt{q} \psi^{2}\left(q^{2}\right) \varphi^{2}(q)}{\varphi^{4}(q)+16 q \psi^{4}\left(q^{2}\right)}\right)^{2},
$$

by (9.25). Similarly, by (9.32) and (9.36),

$$
\beta[q]:=\beta=\left(\frac{8 q^{\frac{3}{2}} \psi^{2}\left(q^{6}\right) \varphi^{2}\left(q^{3}\right)}{\varphi^{4}\left(q^{3}\right)+16 q^{3} \psi^{4}\left(q^{6}\right)}\right)^{2}=\alpha\left[q^{3}\right],
$$

by $(9.37)$.

Hence, from (9.36), (9.37), Lemma 9.14, (9.38), (9.35), and (9.34),

$$
\begin{aligned}
\sqrt{27} & -18 p-p^{2} \\
2 & F_{1}\left(\frac{1}{4}, \frac{3}{4} ; 1 ; \alpha\right) \\
& =\frac{p^{\frac{3}{4}}}{q^{\frac{3}{4}} \psi^{2}\left(q^{3}\right)}\left\{\varphi^{4}\left(q^{3}\right)+16 q^{3} \psi^{4}\left(q^{6}\right)\right\}^{\frac{1}{2}}\left\{\varphi^{4}(q)+16 q \psi^{4}\left(q^{2}\right)\right\}^{\frac{1}{2}} \\
& =\frac{p^{\frac{3}{4}}}{q^{\frac{3}{4}} \psi^{2}\left(q^{3}\right)}{ }_{2} F_{1}\left(\frac{1}{4}, \frac{3}{4} ; 1 ; \beta\right) \frac{q^{\frac{1}{4}} \psi^{2}(q)}{p^{\frac{1}{4}}} \sqrt{3+6 p-p^{2}} \\
& =\frac{\sqrt{p} \psi^{2}(q)}{\sqrt{q} \psi^{2}\left(q^{3}\right)} \sqrt{3+6 p-p^{2}}{ }_{2} F_{1}\left(\frac{1}{4}, \frac{3}{4} ; 1 ; \beta\right) \\
& =3 \sqrt{3+6 p-p^{2}}{ }_{2} F_{1}\left(\frac{1}{4}, \frac{3}{4} ; 1 ; \beta\right) .
\end{aligned}
$$

Thus, (9.33) has been proved.

Lastly, it is easily checked that $\alpha=: \alpha(p)$ and $\beta=: \beta(p)$ are monotonically increasing functions of $p$ on $(0,1)$. Since $\alpha(0)=0=\beta(0)$ and $\alpha(1)=1=$ $\beta(1),(9.33)$ is valid for $0 \leq p<1$.

\section{Modular EQUATIONS IN THE THEORY OF SIGNATURE 4}

Page 261 in Ramanujan's second notebook is devoted to modular equations in the theory of signature 4. In each case, our proofs rely on (9.7). Thus, we shall employ modular equations from Chapters 19 and 20 of the second notebook [35], [5] and convert them via the transformations (10.1)

$$
\alpha \mapsto \frac{2 \sqrt{\alpha}}{1+\sqrt{\alpha}}, \quad 1-\alpha \mapsto \frac{1-\sqrt{\alpha}}{1+\sqrt{\alpha}}, \quad \beta \mapsto \frac{2 \sqrt{\beta}}{1+\sqrt{\beta}}, \quad 1-\beta \mapsto \frac{1-\sqrt{\beta}}{1+\sqrt{\beta}} .
$$

Theorem 10.1 (p. 261). If $\beta$ has degree 3, then

$$
(\alpha \beta)^{\frac{1}{2}}+\{(1-\alpha)(1-\beta)\}^{\frac{1}{2}}+4\{\alpha \beta(1-\alpha)(1-\beta)\}^{\frac{1}{4}}=1 .
$$


Proof. From Entry 5(ii) of Chapter 19 [5, p. 230],

$$
(\alpha \beta)^{\frac{1}{4}}+\{(1-\alpha)(1-\beta)\}^{\frac{1}{4}}=1 .
$$

By $(10.1),(10.3)$ is transformed from the classical theory to

$$
\frac{\sqrt{2}(\alpha \beta)^{\frac{1}{8}}}{\{(1+\sqrt{\alpha})(1+\sqrt{\beta})\}^{\frac{1}{4}}}+\left\{\frac{(1-\sqrt{\alpha})(1-\sqrt{\beta})}{(1+\sqrt{\alpha})(1+\sqrt{\beta})}\right\}^{\frac{1}{4}}=1
$$

in the theory of signature 4 , or

$$
\sqrt{2}(\alpha \beta)^{\frac{1}{8}}=\{(1+\sqrt{\alpha})(1+\sqrt{\beta})\}^{\frac{1}{4}}-\{(1-\sqrt{\alpha})(1-\sqrt{\beta})\}^{\frac{1}{4}} .
$$

Squaring both sides yields

$$
2(\alpha \beta)^{\frac{1}{4}}+2\{(1-\alpha)(1-\beta)\}^{\frac{1}{4}}=\{(1+\sqrt{\alpha})(1+\sqrt{\beta})\}^{\frac{1}{2}}+\{(1-\sqrt{\alpha})(1-\sqrt{\beta})\}^{\frac{1}{2}} .
$$

Squaring each side once again, we find that

$$
\begin{aligned}
2(\alpha \beta)^{\frac{1}{2}} & +2\{(1-\alpha)(1-\beta)\}^{\frac{1}{2}}+4\{\alpha \beta(1-\alpha)(1-\beta)\}^{\frac{1}{4}} \\
& =1+(\alpha \beta)^{\frac{1}{2}}+\{(1-\alpha)(1-\beta)\}^{\frac{1}{2}} .
\end{aligned}
$$

Collecting terms, we deduce (10.2).

Theorem 10.2 (p. 261). If $\beta$ has degree 5 , then

$$
\begin{aligned}
(\alpha \beta)^{\frac{1}{2}}+ & \{(1-\alpha)(1-\beta)\}^{\frac{1}{2}} \\
+ & 8\{\alpha \beta(1-\alpha)(1-\beta)\}^{\frac{1}{6}}\left((\alpha \beta)^{\frac{1}{6}}+\{(1-\alpha)(1-\beta)\}^{\frac{1}{6}}\right)=1 .
\end{aligned}
$$

Proof. By Entry 13(i) in Chapter 19 of Ramanujan's second notebook [5, p. 280],

$$
(\alpha \beta)^{\frac{1}{2}}+\{(1-\alpha)(1-\beta)\}^{\frac{1}{2}}+2\{16 \alpha \beta(1-\alpha)(1-\beta)\}^{\frac{1}{6}}=1 .
$$

Transforming (10.5) by (10.1) and simplifying, we find that, in the theory of signature 4 ,

$$
\begin{aligned}
2(\alpha \beta)^{\frac{1}{4}} & +2\{64 \sqrt{\alpha \beta}(1-\alpha)(1-\beta)\}^{\frac{1}{6}} \\
& =\{(1+\sqrt{\alpha})(1+\sqrt{\beta})\}^{\frac{1}{2}}-\{(1-\sqrt{\alpha})(1-\sqrt{\beta})\}^{\frac{1}{2}} .
\end{aligned}
$$

Squaring each side and collecting terms, we deduce that

$$
\begin{aligned}
& 2(\alpha \beta)^{\frac{1}{2}}+4\{64 \sqrt{\alpha \beta}(1-\alpha)(1-\beta)\}^{\frac{1}{3}}+8(\alpha \beta)^{\frac{1}{3}}\{64(1-\alpha)(1-\beta)\}^{\frac{1}{6}} \\
& =2-2\{(1-\alpha)(1-\beta)\}^{\frac{1}{2}} .
\end{aligned}
$$

Further simplification easily yields (10.4).

Theorem 10.3 (p. 261). If $\beta$ has degree 7, then

$$
\begin{aligned}
(\alpha \beta)^{\frac{1}{2}} & +\{(1-\alpha)(1-\beta)\}^{\frac{1}{2}}+20\{\alpha \beta(1-\alpha)(1-\beta)\}^{\frac{1}{4}} \\
& +8 \sqrt{2}\{\alpha \beta(1-\alpha)(1-\beta)\}^{\frac{1}{8}}\left((\alpha \beta)^{\frac{1}{4}}+\{(1-\alpha)(1-\beta)\}^{\frac{1}{4}}\right)=1 .
\end{aligned}
$$


Proof. From Entry 19(i) in Chapter 19 of Ramanujan's second notebook [5, p. 314],

$$
(\alpha \beta)^{\frac{1}{8}}+\{(1-\alpha)(1-\beta)\}^{\frac{1}{8}}=1 .
$$

Using (10.1) to transform (10.7) into the theory of signature 4 , we find that

$$
(16 \alpha \beta)^{\frac{1}{16}}=\{(1+\sqrt{\alpha})(1+\sqrt{\beta})\}^{\frac{1}{8}}-\{(1-\sqrt{\alpha})(1-\sqrt{\beta})\}^{\frac{1}{8}} .
$$

Squaring both sides, we deduce that

$$
\begin{aligned}
& (16 \alpha \beta)^{\frac{1}{8}}+2\{(1-\alpha)(1-\beta)\}^{\frac{1}{8}} \\
& \quad=\{(1+\sqrt{\alpha})(1+\sqrt{\beta})\}^{\frac{1}{4}}+\{(1-\sqrt{\alpha})(1-\sqrt{\beta})\}^{\frac{1}{4}} .
\end{aligned}
$$

Squaring again and simplifying slightly, we find that

$$
\begin{aligned}
&(16 \alpha \beta)^{\frac{1}{4}}+2\{(1-\alpha)(1-\beta)\}^{\frac{1}{4}}+4\{16 \alpha \beta(1-\alpha)(1-\beta)\}^{\frac{1}{8}} \\
&=\{(1+\sqrt{\alpha})(1+\sqrt{\beta})\}^{\frac{1}{2}}+\{(1-\sqrt{\alpha})(1-\sqrt{\beta})\}^{\frac{1}{2}} .
\end{aligned}
$$

Squaring one more time, we finally deduce that

$$
\begin{aligned}
4(\alpha \beta)^{\frac{1}{2}} & +4\{(1-\alpha)(1-\beta)\}^{\frac{1}{2}}+32\{\alpha \beta(1-\alpha)(1-\beta)\}^{\frac{1}{4}} \\
& +8\{\alpha \beta(1-\alpha)(1-\beta)\}^{\frac{1}{4}}+16(\alpha \beta)^{\frac{1}{4}}\{16 \alpha \beta(1-\alpha)(1-\beta)\}^{\frac{1}{8}} \\
& +16\{(1-\alpha)(1-\beta)\}^{\frac{1}{4}}\{16 \alpha \beta(1-\alpha)(1-\beta)\}^{\frac{1}{8}} \\
= & +2(\alpha \beta)^{\frac{1}{2}}+2\{(1-\alpha)(1-\beta)\}^{\frac{1}{2}} .
\end{aligned}
$$

Collecting terms and dividing both sides by 2 , we complete the proof of (10.6).

Theorem 10.4 (p. 261). If $\beta$ has degree 11 , then

$$
\begin{aligned}
(\alpha \beta)^{\frac{1}{2}}+ & \{(1-\alpha)(1-\beta)\}^{\frac{1}{2}}+68\{\alpha \beta(1-\alpha)(1-\beta)\}^{\frac{1}{4}} \\
& +16\{\alpha \beta(1-\alpha)(1-\beta)\}^{\frac{1}{12}}\left((\alpha \beta)^{\frac{1}{3}}+\{(1-\alpha)(1-\beta)\}^{\frac{1}{3}}\right) \\
& +48\{\alpha \beta(1-\alpha)(1-\beta)\}^{\frac{1}{6}}\left((\alpha \beta)^{\frac{1}{6}}+\{(1-\alpha)(1-\beta)\}^{\frac{1}{6}}\right)=1 .
\end{aligned}
$$

Proof. By Entry 7(i) of Chapter 20 [5, p. 363],

$$
(\alpha \beta)^{\frac{1}{4}}+\{(1-\alpha)(1-\beta)\}^{\frac{1}{4}}+2\{16 \alpha \beta(1-\alpha)(1-\beta)\}^{\frac{1}{12}}=1 .
$$

Transforming (10.9) into an equality in the theory of signature 4 , we find that

$$
\begin{aligned}
& (4 \sqrt{\alpha \beta})^{\frac{1}{4}}+2\{64 \sqrt{\alpha \beta}(1-\alpha)(1-\beta)\}^{\frac{1}{12}} \\
& \quad=\{(1+\sqrt{\alpha})(1+\sqrt{\beta})\}^{\frac{1}{4}}-\{(1-\sqrt{\alpha})(1-\sqrt{\beta})\}^{\frac{1}{4}} .
\end{aligned}
$$

Squaring both sides and simplifying slightly, we deduce that

$$
\begin{aligned}
2(\alpha \beta)^{\frac{1}{4}} & +2\{(1-\alpha)(1-\beta)\}^{\frac{1}{4}}+8(\alpha \beta)^{\frac{1}{12}}\{(1-\alpha)(1-\beta)\}^{\frac{1}{6}} \\
& +8(\alpha \beta)^{\frac{1}{6}}\{(1-\alpha)(1-\beta)\}^{\frac{1}{12}} \\
& =\{(1+\sqrt{\alpha})(1+\sqrt{\beta})\}^{\frac{1}{2}}+\{(1-\sqrt{\alpha})(1-\sqrt{\beta})\}^{\frac{1}{2}} .
\end{aligned}
$$


Squaring both sides again, we see that

$$
\begin{aligned}
4(\alpha \beta)^{\frac{1}{2}} & +4\{(1-\alpha)(1-\beta)\}^{\frac{1}{12}}+64(\alpha \beta)^{\frac{1}{6}}\{(1-\alpha)(1-\beta)\}^{\frac{1}{3}} \\
& +64(\alpha \beta)^{\frac{1}{3}}\{(1-\alpha)(1-\beta)\}^{\frac{1}{6}}+8\{\alpha \beta(1-\alpha)(1-\beta)\}^{\frac{1}{4}} \\
& +32(\alpha \beta)^{\frac{1}{3}}\{(1-\alpha)(1-\beta)\}^{\frac{1}{6}}+32(\alpha \beta)^{\frac{5}{12}}\{(1-\alpha)(1-\beta)\}^{\frac{1}{12}} \\
& +32(\alpha \beta)^{\frac{1}{12}}\{(1-\alpha)(1-\beta)\}^{\frac{5}{12}}+32(\alpha \beta)^{\frac{1}{6}}\{(1-\alpha)(1-\beta)\}^{\frac{1}{3}} \\
& +128\{\alpha \beta(1-\alpha)(1-\beta)\}^{\frac{1}{4}} \\
= & 2+2(\alpha \beta)^{\frac{1}{2}}+2\{(1-\alpha)(1-\beta)\}^{\frac{1}{2}} .
\end{aligned}
$$

Collecting terms and dividing both sides by 2 , we complete the proof.

The last six entries on page 261 in Ramanujan's second notebook give formulas for multipliers. By (9.1),

$$
\begin{aligned}
m(4) & =\frac{{ }_{2} F_{1}\left(\frac{1}{4}, \frac{3}{4} ; 1 ; \alpha\right)}{{ }_{2} F_{1}\left(\frac{1}{4}, \frac{3}{4} ; 1 ; \beta\right)} \\
& =\left(\frac{1+\sqrt{\beta}}{1+\sqrt{\alpha}}\right)^{\frac{1}{2}} \frac{{ }_{2} F_{1}\left(\frac{1}{2}, \frac{1}{2} ; 1 ; \frac{2 \sqrt{\alpha}}{1+\sqrt{\alpha}}\right)}{{ }_{2} F_{1}\left(\frac{1}{2}, \frac{1}{2} ; 1 ; \frac{2 \sqrt{\beta}}{1+\sqrt{\beta}}\right)} .
\end{aligned}
$$

Thus, to obtain formulas for multipliers in the theory of signature 4 , take formulas from the classical theory, replace $m$ by $\left(\frac{1+\sqrt{\alpha}}{1+\sqrt{\beta}}\right)^{\frac{1}{2}} m(4)$, and utilize the transformations in (10.1).

Theorem 10.5 (p. 261). The multiplier for degree 3 is given by

$$
m^{2}(4)=\left(\frac{\beta}{\alpha}\right)^{\frac{1}{2}}+\left(\frac{1-\beta}{1-\alpha}\right)^{\frac{1}{2}}-\frac{9}{m^{2}(4)}\left(\frac{\beta(1-\beta)}{\alpha(1-\alpha)}\right)^{\frac{1}{2}} .
$$

Proof. From Entry 5(vii) in Chapter 19 [5, p. 230],

$$
m^{2}=\left(\frac{\beta}{\alpha}\right)^{\frac{1}{2}}+\left(\frac{1-\beta}{1-\alpha}\right)^{\frac{1}{2}}-\left(\frac{\beta(1-\beta)}{\alpha(1-\alpha)}\right)^{\frac{1}{2}}
$$

and

$$
\frac{9}{m^{2}}=\left(\frac{\alpha}{\beta}\right)^{\frac{1}{2}}+\left(\frac{1-\alpha}{1-\beta}\right)^{\frac{1}{2}}-\left(\frac{\alpha(1-\alpha)}{\beta(1-\beta)}\right)^{\frac{1}{2}} .
$$

Using (10.1) and (10.10), we convert (10.12) into an equality in the theory of signature 4 , namely,

$$
\begin{aligned}
\frac{1+\sqrt{\alpha}}{1+\sqrt{\beta}} m^{2}(4)= & \left(\frac{\sqrt{\beta}(1+\sqrt{\alpha})}{\sqrt{\alpha}(1+\sqrt{\beta})}\right)^{\frac{1}{2}}+\left(\frac{(1-\sqrt{\beta})(1+\sqrt{\alpha})}{(1+\sqrt{\beta})(1-\sqrt{\alpha})}\right)^{\frac{1}{2}} \\
& -\left(\frac{\sqrt{\beta}(1-\sqrt{\beta})(1+\sqrt{\alpha})^{2}}{\sqrt{\alpha}(1-\sqrt{\alpha})(1+\sqrt{\beta})^{2}}\right)^{\frac{1}{2}}
\end{aligned}
$$


or, upon rearrangement,

$$
m^{2}(4)-\left(\frac{1-\beta}{1-\alpha}\right)^{\frac{1}{2}}=\left(\frac{\sqrt{\beta}(1+\sqrt{\beta})}{\sqrt{\alpha}(1+\sqrt{\alpha})}\right)^{\frac{1}{2}}-\left(\frac{\sqrt{\beta}(1-\sqrt{\beta})}{\sqrt{\alpha}(1-\sqrt{\alpha})}\right)^{\frac{1}{2}} .
$$

By (10.13), (10.14), and symmetry,

$$
\frac{9}{m^{2}(4)}-\left(\frac{1-\alpha}{1-\beta}\right)^{\frac{1}{2}}=\left(\frac{\sqrt{\alpha}(1+\sqrt{\alpha})}{\sqrt{\beta}(1+\sqrt{\beta})}\right)^{\frac{1}{2}}-\left(\frac{\sqrt{\alpha}(1-\sqrt{\alpha})}{\sqrt{\beta}(1-\sqrt{\beta})}\right)^{\frac{1}{2}} .
$$

Multiplying both sides of $(10.15)$ by $\left(\frac{\beta(1-\beta)}{\alpha(1-\alpha)}\right)^{\frac{1}{2}}$, we find that (10.16)

$$
\frac{9}{m^{2}(4)}\left(\frac{\beta(1-\beta)}{\alpha(1-\alpha)}\right)^{\frac{1}{2}}-\left(\frac{\beta}{\alpha}\right)^{\frac{1}{2}}=\left(\frac{\sqrt{\beta}(1-\sqrt{\beta})}{\sqrt{\alpha}(1-\sqrt{\alpha})}\right)^{\frac{1}{2}}-\left(\frac{\sqrt{\beta}(1+\sqrt{\beta})}{\sqrt{\alpha}(1+\sqrt{\alpha})}\right)^{\frac{1}{2}} .
$$

Comparing (10.14) and (10.16), we arrive at (10.11).

Theorem 10.6 (p. 261). If $m(4)$ is the multiplier of degree 5 , then

$$
m(4)=\left(\frac{\beta}{\alpha}\right)^{\frac{1}{4}}+\left(\frac{1-\beta}{1-\alpha}\right)^{\frac{1}{4}}-\frac{5}{m(4)}\left(\frac{\beta(1-\beta)}{\alpha(1-\alpha)}\right)^{\frac{1}{4}} \text {. }
$$

Proof. By Entry 13(xii) in Chapter 19 [5, pp. 281-282],

$$
m=\left(\frac{\beta}{\alpha}\right)^{\frac{1}{4}}+\left(\frac{1-\beta}{1-\alpha}\right)^{\frac{1}{4}}-\left(\frac{\beta(1-\beta)}{\alpha(1-\alpha)}\right)^{\frac{1}{4}}
$$

and

$$
\frac{5}{m}=\left(\frac{\alpha}{\beta}\right)^{\frac{1}{4}}+\left(\frac{1-\alpha}{1-\beta}\right)^{\frac{1}{4}}-\left(\frac{\alpha(1-\alpha)}{\beta(1-\beta)}\right)^{\frac{1}{4}} .
$$

Transforming (10.18) into the theory of signature 4 via (10.1) and (10.10), we find, after a slight amount of rearrangement, that

$$
m(4)=\left(\frac{\sqrt{\beta}(1+\sqrt{\beta})}{\sqrt{\alpha}(1+\sqrt{\alpha})}\right)^{\frac{1}{4}}+\left(\frac{1-\beta}{1-\alpha}\right)^{\frac{1}{4}}-\left(\frac{\sqrt{\beta}(1-\sqrt{\beta})}{\sqrt{\alpha}(1-\sqrt{\alpha})}\right)^{\frac{1}{4}} .
$$

From (10.19) and symmetry,

$$
\frac{5}{m(4)}=\left(\frac{\sqrt{\alpha}(1+\sqrt{\alpha})}{\sqrt{\beta}(1+\sqrt{\beta})}\right)^{\frac{1}{4}}+\left(\frac{1-\alpha}{1-\beta}\right)^{\frac{1}{4}}-\left(\frac{\sqrt{\alpha}(1-\sqrt{\alpha})}{\sqrt{\beta}(1-\sqrt{\beta})}\right)^{\frac{1}{4}} .
$$

Multiplying both sides of $(10.21)$ by $\left(\frac{\beta(1-\beta)}{\alpha(1-\alpha)}\right)^{\frac{1}{4}}$ and comparing the result with (10.20), we readily deduce $(10.17)$.

Theorem 10.7 (p. 261). Let $m(4)$ denote the multiplier of degree 9. Then

$$
\sqrt{m(4)}=\left(\frac{\beta}{\alpha}\right)^{\frac{1}{8}}+\left(\frac{1-\beta}{1-\alpha}\right)^{\frac{1}{8}}-\frac{3}{\sqrt{m(4)}}\left(\frac{\beta(1-\beta)}{\alpha(1-\alpha)}\right)^{\frac{1}{8}} \text {. }
$$


Proof. The proof is almost identical to the two previous proofs. By Entries $3(x),(x i)$, respectively, in Chapter 20 [5, p. 352],

$$
\sqrt{m}=\left(\frac{\beta}{\alpha}\right)^{\frac{1}{8}}+\left(\frac{1-\beta}{1-\alpha}\right)^{\frac{1}{8}}-\left(\frac{\beta(1-\beta)}{\alpha(1-\alpha)}\right)^{\frac{1}{8}}
$$

and

$$
\frac{3}{\sqrt{m}}=\left(\frac{\alpha}{\beta}\right)^{\frac{1}{8}}+\left(\frac{1-\alpha}{1-\beta}\right)^{\frac{1}{8}}-\left(\frac{\alpha(1-\alpha)}{\beta(1-\beta)}\right)^{\frac{1}{8}} .
$$

The transforming of (10.23) and (10.24) via (10.1) and (10.10) yields the equalities

$$
\sqrt{m(4)}=\left(\frac{\sqrt{\beta}(1+\sqrt{\beta})}{\sqrt{\alpha}(1+\sqrt{\alpha})}\right)^{\frac{1}{8}}+\left(\frac{1-\beta}{1-\alpha}\right)^{\frac{1}{8}}-\left(\frac{\sqrt{\beta}(1-\sqrt{\beta})}{\sqrt{\alpha}(1-\sqrt{\alpha})}\right)^{\frac{1}{8}}
$$

and

$$
\frac{3}{\sqrt{m(4)}}=\left(\frac{\sqrt{\alpha}(1+\sqrt{\alpha})}{\sqrt{\beta}(1+\sqrt{\beta})}\right)^{\frac{1}{8}}+\left(\frac{1-\alpha}{1-\beta}\right)^{\frac{1}{8}}-\left(\frac{\sqrt{\alpha}(1-\sqrt{\alpha})}{\sqrt{\beta}(1-\sqrt{\beta})}\right)^{\frac{1}{8}}
$$

respectively. A multiplication of $(10.26)$ by $\left(\frac{\beta(1-\beta)}{\alpha(1-\alpha)}\right)^{\frac{1}{8}}$ and a comparison of the resulting equality with (10.25) gives (10.22).

Theorem 10.8 (p. 261). If $m(4)$ denotes the multiplier of degree 7 , then

$$
\begin{aligned}
m^{2}(4)= & \left(\frac{\beta}{\alpha}\right)^{\frac{1}{2}}+\left(\frac{1-\beta}{1-\alpha}\right)^{\frac{1}{2}}-\frac{49}{m^{2}(4)}\left(\frac{\beta(1-\beta)}{\alpha(1-\alpha)}\right)^{\frac{1}{2}} \\
& -8\left(\frac{\beta(1-\beta)}{\alpha(1-\alpha)}\right)^{\frac{1}{6}}\left\{\left(\frac{\beta}{\alpha}\right)^{\frac{1}{6}}+\left(\frac{1-\beta}{1-\alpha}\right)^{\frac{1}{6}}\right\} .
\end{aligned}
$$

Proof. By Entry 19(v) in Chapter 19 [5, p. 314],

$$
m^{2}=\left(\frac{\beta}{\alpha}\right)^{\frac{1}{2}}+\left(\frac{1-\beta}{1-\alpha}\right)^{\frac{1}{2}}-\left(\frac{\beta(1-\beta)}{\alpha(1-\alpha)}\right)^{\frac{1}{2}}-8\left(\frac{\beta(1-\beta)}{\alpha(1-\alpha)}\right)^{\frac{1}{3}}
$$

and

$$
\frac{49}{m^{2}}=\left(\frac{\alpha}{\beta}\right)^{\frac{1}{2}}+\left(\frac{1-\alpha}{1-\beta}\right)^{\frac{1}{2}}-\left(\frac{\alpha(1-\alpha)}{\beta(1-\beta)}\right)^{\frac{1}{2}}-8\left(\frac{\alpha(1-\alpha)}{\beta(1-\beta)}\right)^{\frac{1}{3}} .
$$

Transforming (10.28) and (10.29) into the theory of signature 4 via (10.1) and (10.10), we find that, after simplification,

$$
\begin{aligned}
m^{2}(4)= & \left(\frac{\sqrt{\beta}(1+\sqrt{\beta})}{\sqrt{\alpha}(1+\sqrt{\alpha})}\right)^{\frac{1}{2}}+\left(\frac{1-\beta}{1-\alpha}\right)^{\frac{1}{2}} \\
& -\left(\frac{\sqrt{\beta}(1-\sqrt{\beta})}{\sqrt{\alpha}(1-\sqrt{\alpha})}\right)^{\frac{1}{2}}-8\left(\frac{\sqrt{\beta}(1-\beta)}{\sqrt{\alpha}(1-\alpha)}\right)^{\frac{1}{3}}
\end{aligned}
$$


and

$$
\begin{aligned}
\frac{49}{m^{2}(4)}= & \left(\frac{\sqrt{\alpha}(1+\sqrt{\alpha})}{\sqrt{\beta}(1+\sqrt{\beta})}\right)^{\frac{1}{2}}+\left(\frac{1-\alpha}{1-\beta}\right)^{\frac{1}{2}} \\
& -\left(\frac{\sqrt{\alpha}(1-\sqrt{\alpha})}{\sqrt{\beta}(1-\sqrt{\beta})}\right)^{\frac{1}{2}}-8\left(\frac{\sqrt{\alpha}(1-\alpha)}{\sqrt{\beta}(1-\beta)}\right)^{\frac{1}{3}},
\end{aligned}
$$

respectively. Multiplying both sides of $(10.31)$ by $\left(\frac{\beta(1-\beta)}{\alpha(1-\alpha)}\right)^{\frac{1}{2}}$, we deduce that

$$
\begin{aligned}
\frac{49}{m^{2}(4)}\left(\frac{\beta(1-\beta)}{\alpha(1-\alpha)}\right)^{\frac{1}{2}}= & \left(\frac{\sqrt{\beta}(1-\sqrt{\beta})}{\sqrt{\alpha}(1-\sqrt{\alpha})}\right)^{\frac{1}{2}}+\left(\frac{\beta}{\alpha}\right)^{\frac{1}{2}} \\
& -\left(\frac{\sqrt{\beta}(1+\sqrt{\beta})}{\sqrt{\alpha}(1+\sqrt{\alpha})}\right)^{\frac{1}{2}}-8\left(\frac{\beta \sqrt{1-\beta}}{\alpha \sqrt{1-\alpha}}\right)^{\frac{1}{3}} .
\end{aligned}
$$

Combining (10.30) and (10.32), we complete the proof of (10.27).

Theorem 10.9 (p. 261). If $m(4)$ denotes the multiplier of degree 13 , then

$$
\begin{aligned}
m(4)= & \left(\frac{\beta}{\alpha}\right)^{\frac{1}{4}}+\left(\frac{1-\beta}{1-\alpha}\right)^{\frac{1}{4}}-\frac{13}{m(4)}\left(\frac{\beta(1-\beta)}{\alpha(1-\alpha)}\right)^{\frac{1}{4}} \\
& -4\left(\frac{\beta(1-\beta)}{\alpha(1-\alpha)}\right)^{\frac{1}{12}}\left\{\left(\frac{\beta}{\alpha}\right)^{\frac{1}{12}}+\left(\frac{1-\beta}{1-\alpha}\right)^{\frac{1}{12}}\right\} .
\end{aligned}
$$

Proof. By Entries 8(iii),(iv) in Chapter 20 of Ramanujan's second notebook [5, p. 376],

$$
m=\left(\frac{\beta}{\alpha}\right)^{\frac{1}{4}}+\left(\frac{1-\beta}{1-\alpha}\right)^{\frac{1}{4}}-\left(\frac{\beta(1-\beta)}{\alpha(1-\alpha)}\right)^{\frac{1}{4}}-4\left(\frac{\beta(1-\beta)}{\alpha(1-\alpha)}\right)^{\frac{1}{6}}
$$

and

$$
\frac{13}{m}=\left(\frac{\alpha}{\beta}\right)^{\frac{1}{4}}+\left(\frac{1-\alpha}{1-\beta}\right)^{\frac{1}{4}}-\left(\frac{\alpha(1-\alpha)}{\beta(1-\beta)}\right)^{\frac{1}{4}}-4\left(\frac{\alpha(1-\alpha)}{\beta(1-\beta)}\right)^{\frac{1}{6}} .
$$

Transforming (10.34) and (10.35) into the system of signature 4 by means of (10.1) and (10.10), we find, after some simplification, that

$$
m(4)=\left(\frac{\sqrt{\beta}(1+\sqrt{\beta})}{\sqrt{\alpha}(1+\sqrt{\alpha})}\right)^{\frac{1}{4}}+\left(\frac{1-\beta}{1-\alpha}\right)^{\frac{1}{4}}-\left(\frac{\sqrt{\beta}(1-\sqrt{\beta})}{\sqrt{\alpha}(1-\sqrt{\alpha})}\right)^{\frac{1}{4}}-4\left(\frac{\sqrt{\beta}(1-\beta)}{\sqrt{\alpha}(1-\alpha)}\right)^{\frac{1}{6}}
$$

and

(10.37)

$$
\frac{13}{m(4)}=\left(\frac{\sqrt{\alpha}(1+\sqrt{\alpha})}{\sqrt{\beta}(1+\sqrt{\beta})}\right)^{\frac{1}{4}}+\left(\frac{1-\alpha}{1-\beta}\right)^{\frac{1}{4}}-\left(\frac{\sqrt{\alpha}(1-\sqrt{\alpha})}{\sqrt{\beta}(1-\sqrt{\beta})}\right)^{\frac{1}{4}}-4\left(\frac{\sqrt{\alpha}(1-\alpha)}{\sqrt{\beta}(1-\beta)}\right)^{\frac{1}{6}},
$$

respectively. Multiplying both sides of $(10.37)$ by $\left(\frac{\beta(1-\beta)}{\alpha(1-\alpha)}\right)^{\frac{1}{4}}$ and combining the resulting equality with (10.36), we finish the proof of (10.33). 
Theorem 10.10 (p. 261). If $m(4)$ denotes the multiplier of degree 25 , then

$$
\begin{aligned}
\sqrt{m(4)}= & \left(\frac{\beta}{\alpha}\right)^{\frac{1}{8}}+\left(\frac{1-\beta}{1-\alpha}\right)^{\frac{1}{8}}-\frac{5}{\sqrt{m(4)}}\left(\frac{\beta(1-\beta)}{\alpha(1-\alpha)}\right)^{\frac{1}{8}} \\
& -2\left(\frac{\beta(1-\beta)}{\alpha(1-\alpha)}\right)^{\frac{1}{24}}\left\{\left(\frac{\beta}{\alpha}\right)^{\frac{1}{24}}+\left(\frac{1-\beta}{1-\alpha}\right)^{\frac{1}{24}}\right\} .
\end{aligned}
$$

Proof. By Entries 15(i),(ii) in Chapter 19 of Ramanujan's second notebook [5, p. 291],

$$
\sqrt{m}=\left(\frac{\beta}{\alpha}\right)^{\frac{1}{8}}+\left(\frac{1-\beta}{1-\alpha}\right)^{\frac{1}{8}}-\left(\frac{\beta(1-\beta)}{\alpha(1-\alpha)}\right)^{\frac{1}{8}}-2\left(\frac{\beta(1-\beta)}{\alpha(1-\alpha)}\right)^{\frac{1}{12}}
$$

and

$$
\frac{5}{\sqrt{m}}=\left(\frac{\alpha}{\beta}\right)^{\frac{1}{8}}+\left(\frac{1-\alpha}{1-\beta}\right)^{\frac{1}{8}}-\left(\frac{\alpha(1-\alpha)}{\beta(1-\beta)}\right)^{\frac{1}{8}}-2\left(\frac{\alpha(1-\alpha)}{\beta(1-\beta)}\right)^{\frac{1}{12}} .
$$

Transforming (10.39) and (10.40) by means of (10.1) and (10.10) into equalities in the theory of signature 4 , we find that

$$
\begin{aligned}
\sqrt{m(4)}= & \left(\frac{\sqrt{\beta}(1+\sqrt{\beta})}{\sqrt{\alpha}(1+\sqrt{\alpha})}\right)^{\frac{1}{8}}+\left(\frac{1-\beta}{1-\alpha}\right)^{\frac{1}{8}} \\
& -\left(\frac{\sqrt{\beta}(1-\sqrt{\beta})}{\sqrt{\alpha}(1-\sqrt{\alpha})}\right)^{\frac{1}{8}}-2\left(\frac{\sqrt{\beta}(1-\beta)}{\sqrt{\alpha}(1-\alpha)}\right)^{\frac{1}{12}}
\end{aligned}
$$

and

$$
\begin{aligned}
\frac{5}{\sqrt{m(4)}}= & \left(\frac{\sqrt{\alpha}(1+\sqrt{\alpha})}{\sqrt{\beta}(1+\sqrt{\beta})}\right)^{\frac{1}{8}}+\left(\frac{1-\alpha}{1-\beta}\right)^{\frac{1}{8}} \\
& -\left(\frac{\sqrt{\alpha}(1-\sqrt{\alpha})}{\sqrt{\beta}(1-\sqrt{\beta})}\right)^{\frac{1}{8}}-2\left(\frac{\sqrt{\alpha}(1-\alpha)}{\sqrt{\beta}(1-\beta)}\right)^{\frac{1}{12}},
\end{aligned}
$$

respectively. Multiplying both sides of $(10.42)$ by $\left(\frac{\beta(1-\beta)}{\alpha(1-\alpha)}\right)^{\frac{1}{8}}$ and combining the resulting equality with (10.41), we finish the proof of (10.38).

\section{THE THEORY FOR SIGNATURE 6}

The most important theorem in this section is Theorem 11.3 below. This result allows us to employ formulas in the classical theory to prove corresponding theorems in the theory of signature 6 . To prove Theorem 11.3, we need the following two results.

Theorem 11.1 (p. 262). If

$$
\alpha=\frac{p(2+p)}{1+2 p} \quad \text { and } \quad \beta=\frac{27 p^{2}(1+p)^{2}}{4\left(1+p+p^{2}\right)^{3}},
$$

then, for $0 \leq p<1$,

$$
\sqrt{1+2 p}{ }_{2} F_{1}\left(\frac{1}{6}, \frac{5}{6} ; 1 ; \beta\right)=\sqrt{1+p+p^{2}}{ }_{2} F_{1}\left(\frac{1}{2}, \frac{1}{2} ; 1 ; \alpha\right) .
$$


Proof. From Erdélyi's treatise [21, p. 114, eq. (42)],

(11.3) ${ }_{2} F_{1}\left(\frac{1}{4}, \frac{1}{4} ; 1 ; z\right)=(1-z / 4)^{-\frac{1}{4}}{ }_{2} F_{1}\left(\frac{1}{12}, \frac{5}{12} ; 1 ;-27 z^{2}(z-4)^{-3}\right)$,

for $z$ sufficiently near the origin. By Example (ii) in Section 33 of Chapter 11 [35], [4, p. 95],

$$
{ }_{2} F_{1}\left(\frac{1}{2}, \frac{1}{2} ; 1 ; z\right)=(1-z)^{-\frac{1}{2}}{ }_{2} F_{1}\left(\frac{1}{4}, \frac{1}{4} ; 1 ;-\frac{4 z}{(1-z)^{2}}\right),
$$

for $|z|$ sufficiently small. Thus, combining (11.3) and (11.4), we find that (11.5)

$$
\begin{aligned}
{ }_{2} F_{1}\left(\frac{1}{2}, \frac{1}{2} ; 1 ; z\right)= & (1-z)^{-\frac{1}{2}}\left(1+\frac{z}{(1-z)^{2}}\right)^{-\frac{1}{4}} \\
& \cdot{ }_{2} F_{1}\left(\frac{1}{12}, \frac{5}{12} ; 1 ; \frac{27 z^{2}}{4(1-z)^{4}}\left(\frac{z}{(1-z)^{2}}+1\right)^{-3}\right) \\
= & \left(1-z+z^{2}\right)^{-\frac{1}{4}}{ }_{2} F_{1}\left(\frac{1}{12}, \frac{5}{12} ; 1 ; \frac{27 z^{2}(1-z)^{2}}{4\left(1-z+z^{2}\right)^{3}}\right) .
\end{aligned}
$$

Next, recall the well-known transformation [21, p. 111, eq. (2)],

$$
{ }_{2} F_{1}\left(\frac{1}{6}, \frac{5}{6} ; 1 ; z\right)={ }_{2} F_{1}\left(\frac{1}{12}, \frac{5}{12} ; 1 ; 4 z(1-z)\right)
$$

for $z$ in some neighborhood of the origin. Examining (11.5) and (11.6) in relation to (11.2), we see that we want to solve the equation

$$
4 x(1-x)=\frac{27 z^{2}(1-z)^{2}}{4\left(1-z+z^{2}\right)^{3}} .
$$

Solving this quadratic equation in $x$ and choosing that root which is near the origin when $z$ is close to 0 , we find that

$$
\begin{aligned}
x & =\frac{1}{2}\left(1-\left\{\frac{4\left(1-z+z^{2}\right)^{3}-27 z^{2}(1-z)^{2}}{4\left(1-z+z^{2}\right)^{3}}\right\}^{\frac{1}{2}}\right) \\
& =\frac{1}{2}\left(1-\frac{(1+z)\left(2-5 z+2 z^{2}\right)}{2\left(1-z+z^{2}\right)^{\frac{3}{2}}}\right) .
\end{aligned}
$$

Thus, by (11.5)-(11.7), we have shown that

$$
\begin{gathered}
{ }_{2} F_{1}\left(\frac{1}{6}, \frac{5}{6} ; 1 ; \frac{1}{2}\left(1-\frac{(1+z)\left(2-5 z+2 z^{2}\right)}{2\left(1-z+z^{2}\right)^{\frac{3}{2}}}\right)\right) \\
=\left(1-z+z^{2}\right)^{\frac{1}{4}}{ }_{2} F_{1}\left(\frac{1}{2}, \frac{1}{2} ; 1 ; z\right) .
\end{gathered}
$$

Now set

$$
z=\frac{p(2+p)}{1+2 p} .
$$

Then elementary calculations give

$$
1-z+z^{2}=\frac{\left(1+p+p^{2}\right)^{2}}{(1+2 p)^{2}}
$$


and

$$
1-\frac{(1+z)\left(2-5 z+2 z^{2}\right)}{2\left(1-z+z^{2}\right)^{\frac{3}{2}}}=\frac{27 p^{2}(1+p)^{2}}{2\left(1+p+p^{2}\right)^{3}}
$$

Using these calculations in (11.8), we deduce (11.2).

Lastly, $\alpha$ and $\beta$ are monotonically increasing functions of $p$ on $[0,1]$ with $\alpha(0)=0=\beta(0)$ and $\alpha(1)=1=\beta(1)$. It follows that (11.2) is valid for $0 \leq p<1$.

Corollary 11.2. Let $\alpha$ and $\beta$ be defined by (11.1). For $0<p \leq 1$,

$$
\begin{aligned}
& \sqrt{1+2 p}{ }_{2} F_{1}\left(\frac{1}{6}, \frac{5}{6} ; 1 ; 1-\beta\right) \\
& \quad=\sqrt{1+p+p^{2}}{ }_{2} F_{1}\left(\frac{1}{2}, \frac{1}{2} ; 1 ; 1-\alpha\right) .
\end{aligned}
$$

Proof. From (11.1),

$$
1-\alpha=\frac{1-p^{2}}{1+2 p} \quad \text { and } \quad 1-\beta=\frac{(1-p)^{2}(1+2 p)^{2}(2+p)^{2}}{4\left(1+p+p^{2}\right)^{3}}
$$

(Recall that $1-\beta$ was previously calculated in (5.35).) Setting

$$
z=\frac{1-p^{2}}{1+2 p}
$$

in (11.8), we complete the proof.

Theorem 11.3. Let $\alpha$ and $\beta$ be defined by (11.1). If $0<p<1$, then

$$
q_{6}=: q_{6}(\beta)=q^{2}(\alpha):=q^{2},
$$

where $q$ denotes the classical base.

Proof. Divide (11.9) by (11.2) to obtain the equality

$$
\frac{{ }_{2} F_{1}\left(\frac{1}{6}, \frac{5}{6} ; 1 ; 1-\beta\right)}{{ }_{2} F_{1}\left(\frac{1}{6}, \frac{5}{6} ; 1 ; \beta\right)}=\frac{{ }_{2} F_{1}\left(\frac{1}{2}, \frac{1}{2} ; 1 ; 1-\alpha\right)}{{ }_{2} F_{1}\left(\frac{1}{2}, \frac{1}{2} ; 1 ; \alpha\right)},
$$

valid for $0<p<1$. From (11.12) and (1.9), we immediately deduce (11.11).

From Theorem 11.1, we also can deduce that

$$
\begin{aligned}
\sqrt{1+2 p} z(6 ; \beta) & :=\sqrt{1+2 p} z(6)=\sqrt{1+p+p^{2}} z(2) \\
& =: \sqrt{1+p+p^{2}} z(2 ; \alpha) .
\end{aligned}
$$

Hence, from (11.1), (11.11), and (11.13), we derive the following principle. Suppose that we have an equality

$$
\Omega\left(q^{2}, z(2 ; \alpha(p))\right)=0
$$


in the classical theory. Then in the theory of signature 6 , we may deduce the corresponding equality

$$
\Omega\left(q_{6},\left(\frac{1+2 p}{1+p+p^{2}}\right)^{\frac{1}{2}} z(6 ; \beta(p))\right)=0 .
$$

We now give some applications of this principle.

Theorem 11.4 (p. 262). We have

$$
M\left(q_{6}\right)=z^{4}(6) .
$$

Proof. By Entry 13(i) in Chapter 17 of Ramanujan's second notebook [5, p. 126], (11.11), and (11.1),

$$
\begin{aligned}
M\left(q_{6}\right)=M\left(q^{2}\right) & =z^{4}\left(1-\alpha+\alpha^{2}\right) \\
& =z^{4}\left(1-\frac{p(2+p)}{1+2 p}+\frac{p^{2}(2+p)^{2}}{(1+2 p)^{2}}\right) \\
& =z^{4}\left(\frac{1+p+p^{2}}{1+2 p}\right)^{2} \\
& =z^{4}(6),
\end{aligned}
$$

by (11.13).

Theorem 11.5 (p. 262). We have

$$
N\left(q_{6}\right)=z^{6}(6)(1-2 \beta) .
$$

Proof. By Entry 13(ii) in Chapter 17 [5, p. 126], (11.11), and (11.1),

$$
\begin{aligned}
N\left(q_{6}\right)=N\left(q^{2}\right) & =z^{6}(1+\alpha)\left(1-\frac{1}{2} \alpha\right)(1-2 \alpha) \\
& =z^{6}\left(1+\frac{p(2+p)}{1+2 p}\right)\left(1-\frac{p(2+p)}{2(1+2 p)}\right)\left(1-\frac{2 p(2+p)}{1+2 p}\right) \\
& =z^{6} \frac{\left(1+4 p+p^{2}\right)\left(2+2 p-p^{2}\right)\left(1-2 p-2 p^{2}\right)}{2(1+2 p)^{3}} \\
& =z^{6} \frac{2\left(1+p+p^{2}\right)^{3}-27 p^{2}(1+p)^{2}}{2(1+2 p)^{3}} \\
& =z^{6} \frac{\left(1+p+p^{2}\right)^{3}}{(1+2 p)^{3}} \frac{2\left(1+p+p^{2}\right)^{3}-27 p^{2}(1+p)^{2}}{2\left(1+p+p^{2}\right)^{3}} \\
& =z^{6}(6)(1-2 \beta),
\end{aligned}
$$

by (11.13) and (11.1).

Theorem 11.6 (p. 262). We have

$$
q_{6}^{\frac{1}{24}} f\left(-q_{6}\right)=\sqrt{z(6)}\left(\frac{\beta(1-\beta)}{432}\right)^{\frac{1}{24}} .
$$


Proof. By Entry 12(iii) in Chapter 17 [5, p. 124], (11.11), (11.13), and (11.1),

$$
\begin{aligned}
q_{6}^{\frac{1}{24}} f\left(-q_{6}\right) & =q^{\frac{1}{12}} f\left(-q^{2}\right)=\sqrt{z} 2^{-\frac{1}{3}}\{\alpha(1-\alpha)\}^{\frac{1}{12}} \\
& =\sqrt{z(6)}\left(\frac{1+2 p}{1+p+p^{2}}\right)^{\frac{1}{4}} 2^{-\frac{1}{3}}\left(\frac{p(2+p)}{1+2 p} \frac{1-p^{2}}{1+2 p}\right)^{\frac{1}{12}} \\
& =\sqrt{z(6)} \frac{\left\{p\left(1-p^{2}\right)(1+2 p)(2+p)\right\}^{\frac{1}{12}}}{2^{\frac{1}{3}}\left(1+p+p^{2}\right)^{\frac{1}{4}}} \\
& =\sqrt{z(6)}\left(\frac{27 p^{2}(1+p)^{2}}{4\left(1+p+p^{2}\right)^{3}} \frac{(1-p)^{2}(1+2 p)^{2}(2+p)^{2}}{4\left(1+p+p^{2}\right)^{3}}\right)^{\frac{1}{24}}\left(\frac{1}{432}\right)^{\frac{1}{24}} \\
& =\sqrt{z(6)}\left(\frac{\beta(1-\beta)}{432}\right)^{\frac{1}{24}},
\end{aligned}
$$

by (11.1) and (11.10).

\section{AN IDENTITY FROM THE FIRST NOTEBOOK AND FURTHER HYPERGEOMETRIC TRANSFORMATIONS}

On page 96 in his first notebook, Ramanujan claims that

$$
\varphi\left(e^{-\frac{\pi y}{\sin (\pi h)}}\right)=\mu \sqrt{{ }_{2} F_{1}(h, 1-h ; 1 ; x)},
$$

where " $\mu$ can be expressed in radicals of $x$ and $h$ " and where

$$
y=\frac{{ }_{2} F_{1}(h, 1-h ; 1 ; 1-x)}{{ }_{2} F_{1}(h, 1-h ; 1 ; x)} .
$$

It is unclear what Ramanujan precisely means by the phrase, " $\mu$ can be expressed in radicals of $x$ and $h$." If Ramanujan means that $\mu$ is contained in some radical extension of the field $\mathbb{Q}(x, h)$, the field of rational functions in $x$ and $h$, then his statement is false for general $h$. We now sketch a proof.

By Corollary (ii) in Section 2 of Chapter 17 in Ramanujan's second notebook $[5, \mathrm{p} .90]$,

$$
\begin{aligned}
& \exp (-\pi y / \sin (\pi h))=x \exp (\psi(h)+\psi(1-h)+2 \gamma) \\
& \quad \times\left\{1+\left(2 h^{2}-2 h+1\right) x+\left(1-\frac{7}{2}\left(h-h^{2}\right)+\frac{13}{4}\left(h-h^{2}\right)^{2}\right) x^{2}+\cdots\right\},
\end{aligned}
$$

where $\psi(x)$ is the logarithmic derivative of the Gamma function. It should be noted that for $h=\frac{1}{2}, \frac{1}{3}, \frac{1}{4}, \frac{1}{6}$ the quantity $\exp (\psi(h)+\psi(1-h)+2 \gamma)$ is a rational number. In fact, from Chapter 8 of Ramanujan's second notebook [3, p.192],

$$
\begin{aligned}
\exp \left(2 \psi\left(\frac{1}{2}\right)+2 \gamma\right) & =\frac{1}{16}, \\
\exp \left(\psi\left(\frac{1}{3}\right)+\psi\left(\frac{2}{3}\right)+2 \gamma\right) & =\frac{1}{27}, \\
\exp \left(\psi\left(\frac{1}{4}\right)+\psi\left(\frac{3}{4}\right)+2 \gamma\right) & =\frac{1}{64},
\end{aligned}
$$

and 


$$
\exp \left(\psi\left(\frac{1}{6}\right)+\psi\left(\frac{5}{6}\right)+2 \gamma\right)=\frac{1}{432} .
$$

However, for $h=\frac{1}{5}$ the quantity is transcendental, for [3, p.192],

$$
\exp \left(\psi\left(\frac{1}{5}\right)+\psi\left(\frac{4}{5}\right)+2 \gamma\right)=5^{-5 / 2}\left(\frac{\sqrt{5}+1}{2}\right)^{-\sqrt{5}},
$$

which is transcendental by the Gelfond-Schneider theorem. If Ramanujan's statement were true for general $h$, this would imply that, for $h=\frac{1}{5}, \mu$ could be expressed in terms of radicals over some algebraic extension field of $\mathbb{Q}$. Now $\sqrt{{ }_{2} F_{1}(h, 1-h ; 1 ; x)}$ and, by (12.3), $\varphi\left(e^{-\frac{\pi y}{\sin (n h)}}\right)$ have Taylor expansions about $x=0$. Thus, (12.1) implies that $\mu$ has a Taylor expansion about $x=0$. But since $\mu$ can be expressed in terms of radicals in $x$, it follows that the coefficients in the Taylor expansion of $\mu$ are algebraic over $\mathbb{Q}$. The function $\sqrt{{ }_{2} F_{1}(h, 1-h ; 1 ; x)}$ has the same property. Hence, by equating coefficients on both sides of $(12.1)$, we deduce that $\exp \left(\psi\left(\frac{1}{5}\right)+\psi\left(\frac{4}{5}\right)+2 \gamma\right)$ is algebraic, which is a contradiction. Hence, Ramanujan's statement is false for general $h$.

However, the statement is true if we restrict the values of $h$ to $\frac{1}{2}, \frac{1}{3}, \frac{1}{4}, \frac{1}{6}$. Let $\mu=\mu(x, h)$. By $(1.15)$,

$$
\mu\left(x, \frac{1}{2}\right)=1 .
$$

We now describe $\mu\left(x, \frac{1}{3}\right)$ in terms of radicals in $x$. Recall that $m=z_{1} / z_{3}$.

Theorem 12.1. For $0<x<1$,

$$
\mu\left(x, \frac{1}{3}\right)=\frac{2 m^{3 / 4}}{\sqrt{m^{2}+6 m-3}},
$$

where

$$
m=\frac{M-\sqrt{(M+2)(M-6)}}{2},
$$

and $M$ is the root of the cubic equation

$$
x=\frac{27(M+2)^{2}}{(M+6)^{3}}
$$

that is greater then 6. More explicitly, $M$ may be given as

$$
\begin{aligned}
M=\frac{3}{x}\left(\sqrt[3]{8 x^{2}-36 x+27+8 i \sqrt{(1-x) x^{3}}}\right. & \\
& \left.+\sqrt[3]{8 x^{2}-36 x+27-8 i \sqrt{(1-x) x^{3}}}+3-2 x\right) .
\end{aligned}
$$

To make explicit which cube root is taken, we rewrite (12.6) as (12.7)

$$
M=\frac{3}{x}\left(2 \sqrt{9-8 x} \cos \left(\frac{1}{3} \arctan \left(8 \sqrt{(1-x) x^{3}}, 8 x^{2}-36 x+27\right)\right)+3-2 x\right),
$$


where $\arctan (\beta, \alpha)$ is that angle $\theta$ such that $-\pi<\theta \leq \pi$ and $\arg (\alpha+i \beta)=\theta$. Proof. By Lemma 2.9 and Theorem 2.10,

$$
\mu\left(\frac{c^{3}(q)}{a^{3}(q)}, \frac{1}{3}\right)=\frac{\varphi(q)}{\sqrt{a(q)}},
$$

for $0<q<1$. By Lemma 2.1,

$$
x:=\frac{c^{3}(q)}{a^{3}(q)}=\frac{27(m+1)^{3}\left(m^{2}-1\right)}{\left(m^{2}+6 m-3\right)^{3}},
$$

and

$$
\mu\left(x, \frac{1}{3}\right)=\frac{\varphi(q)}{\sqrt{a(q)}}=\frac{2 m^{3 / 4}}{\sqrt{m^{2}+6 m-3}},
$$

which is (12.4). From (3.12), (3.15), and the definition of $m$, we observe that $m=m(q)$ maps the interval $(0,1)$ monotonically onto the interval $(1,3)$, and if we view (12.8) as defining $x$ in terms of $m, x=x(m)$ maps the interval $(1,3)$ monotonically onto the interval $(0,1)$. We conclude that $x=\frac{c^{3}(q)}{a^{3}(q)}$ maps the interval $(0,1)$ monotonically onto itself. We see that equation $(12.8)$ is solvable by rewriting it as

$$
x=\frac{c^{3}(q)}{a^{3}(q)}=\frac{27(M+2)^{2}}{(M+6)^{3}}=: x(M),
$$

where

$$
M=\frac{m^{2}+3}{m-1} .
$$

In terms of $q$-series, $M$ is the sum of two theta-products, namely,

$$
M=m+\frac{m+3}{m-1}=\frac{\varphi^{2}(q)}{\varphi^{2}\left(q^{3}\right)}+\frac{\psi^{2}\left(q^{2}\right)}{q \psi^{2}\left(q^{6}\right)},
$$

by (1.17) and (2.13)-(2.15). Since $1<m<3$, we see that $M>6$. We may solve the quadratic equation (12.11) for $M$. The solution is given above in (12.5). We have taken the negative square root since $1<m<3$. Since (12.10) is a cubic equation in $M$, we may solve it in terms of radicals. Note that $x(-3)=x(6)=1, x(-2)=0$, and

$$
\frac{d x}{d M}=-\frac{27(M+2)(M-6)}{(M+6)^{4}} .
$$

Thus, $x(M)$ decreases from 1 to 0 on $(-3,-2)$, increases from 0 to 1 on $(-2,6)$, and decreases from 1 to 0 on $(6, \infty)$. Therefore, if $0<x<1$, the equation $(12.10)$ has three real roots, and each of the intervals $(-3,-2)$, $(-2,6)$, and $(6, \infty)$ contains exactly one root. Since $M>6$, we must take $M$ to be the largest of the roots. The solution is given in terms of radicals in (12.6) above and more explicitly in (12.7).

For $h=\frac{1}{4}, \mu$ has a particularly simple form. 
Theorem 12.2. For $0<x<1$,

$$
\mu\left(x, \frac{1}{4}\right)=\left(\frac{1+\sqrt{1-x}}{2}\right)^{\frac{1}{4}} .
$$

Proof. With $h=\frac{1}{r}$, we recall that

$$
q_{r}=q_{r}(x)=\exp ((-\pi y / \sin (\pi h)),
$$

where $y$ is defined by (12.2). We shall prove that

$$
q_{4}\left(\left(\frac{8 \sqrt{q} \psi^{2}\left(q^{2}\right) \varphi^{2}(q)}{\varphi^{4}(q)+16 q \psi^{4}\left(q^{2}\right)}\right)^{2}\right)=q .
$$

If $0<q<1$, then

$$
q=\exp (-\pi t)
$$

for some unique $t>0$. Set

$$
x:=\left(\frac{8 \sqrt{q} \psi^{2}\left(q^{2}\right) \varphi^{2}(q)}{\varphi^{4}(q)+16 q \psi^{4}\left(q^{2}\right)}\right)^{2} .
$$

In view of (12.15), we consider $x=x(t)$ as a function of $t$. Define

$$
z(t)=\varphi^{4}(q)+16 q \psi^{4}\left(q^{2}\right) .
$$

Then, by Lemma 9.14,

$$
{ }_{2} F_{1}\left(\frac{1}{4}, \frac{3}{4} ; 1 ; x(t)\right)=\sqrt{z(t)} .
$$

We shall prove that

$$
x(2 / t)=1-x(t)
$$

and

$$
z(2 / t)=\frac{1}{2} t^{2} z(t) .
$$

We first show that (12.19) and (12.20) imply (12.14). To that end, by (12.2), (12.19), (12.18), and (12.20),

$$
\begin{aligned}
y & =\frac{{ }_{2} F_{1}\left(\frac{1}{4}, \frac{3}{4} ; 1 ; 1-x(t)\right)}{{ }_{2} F_{1}\left(\frac{1}{4}, \frac{3}{4} ; 1 ; x(t)\right)} \\
& =\frac{{ }_{2} F_{1}\left(\frac{1}{4}, \frac{3}{4} ; 1 ; x(2 / t)\right)}{{ }_{2} F_{1}\left(\frac{1}{4}, \frac{3}{4} ; 1 ; x(t)\right)}=\sqrt{\frac{z(2 / t)}{z(t)}}=\frac{t}{\sqrt{2}} .
\end{aligned}
$$

Hence, by (12.13), (12.21), and (12.15),

$$
q_{4}(x(t))=\exp (-\pi \sqrt{2} y)=\exp (-\pi t)=q,
$$

which is (12.14). It remains to prove (12.19) and (12.20).

We shall need the transformation formulas [12, p. 40, eqs. (2.3.1)-(2.3.2)]

$$
2 e^{-\pi /(4 t)} \psi\left(e^{-2 \pi / t}\right)=\sqrt{t} \varphi\left(-e^{-\pi t}\right)
$$

and

$$
\varphi\left(e^{-\pi / t}\right)=\sqrt{t} \varphi\left(e^{-\pi t}\right) .
$$


Thus, by (12.23), (12.22), (2.1), (9.31), and (9.25),

$$
\begin{aligned}
z(2 / t) & =\frac{1}{4} t^{2}\left(\varphi^{4}(\sqrt{q})+\varphi^{4}(-\sqrt{q})\right) \\
& =\frac{1}{4} t^{2}\left(2 \varphi^{4}(\sqrt{q})-\left(\varphi^{4}(\sqrt{q})-\varphi^{4}(-\sqrt{q})\right)\right) \\
& =\frac{1}{4} t^{2}\left(2 \varphi^{4}(\sqrt{q})-16 \sqrt{q} \psi^{4}(q)\right) \\
& =\frac{1}{4} t^{2}\left(2\left(\varphi^{2}(q)+4 \sqrt{q} \psi^{2}\left(q^{2}\right)\right)^{2}-16 \sqrt{q} \psi^{2}\left(q^{2}\right) \varphi^{2}(q)\right) \\
& =\frac{1}{2} t^{2}\left(\varphi^{4}(q)+16 q \psi^{4}\left(q^{2}\right)\right) \\
& =\frac{1}{2} t^{2} z(t),
\end{aligned}
$$

by (12.17). This proves (12.20).

Next, define

$$
w(t):=64 q \psi^{4}\left(q^{2}\right) \varphi^{4}(q)=64 q \psi^{8}\left(e^{-\pi t}\right),
$$

by $(9.25)$. Hence, by (12.16) and (12.17),

$$
x(t)=\frac{w(t)}{z^{2}(t)} .
$$

Now, by (12.24), (12.22), and (2.1),

$$
\begin{aligned}
w(2 / t) & =\frac{1}{4} t^{4} \varphi^{8}(-q) \\
& =\frac{1}{4} t^{4}\left(\varphi^{4}(q)-16 q \psi^{4}\left(q^{2}\right)\right)^{2} \\
& =\frac{1}{4} t^{4}\left(\left(\varphi^{4}(q)+16 q \psi^{4}\left(q^{2}\right)\right)^{2}-64 q \psi^{4}\left(q^{2}\right) \varphi^{4}(q)\right) \\
& =\frac{1}{4} t^{4}\left(z^{2}(t)-w(t)\right),
\end{aligned}
$$

by (12.17) and (12.24). Hence, by (12.25), (12.26), and (12.20),

$$
x(2 / t)=\frac{w(2 / t)}{z^{2}(2 / t)}=\frac{z^{2}(t)-w(t)}{z^{2}(t)}=1-x(t),
$$

which is (12.19).

Hence, from (12.1) and (12.14),

$$
\mu\left(\left(\frac{8 \sqrt{q} \psi^{2}\left(q^{2}\right) \varphi^{2}(q)}{\varphi^{4}(q)+16 q \psi^{4}\left(q^{2}\right)}\right)^{2}, \frac{1}{4}\right)=\frac{\varphi(q)}{\left(\varphi^{4}(q)+16 q \psi^{4}\left(q^{2}\right)\right)^{\frac{1}{4}}} .
$$

If we let

$$
\alpha=\alpha(q)=\frac{16 q \psi^{4}\left(q^{2}\right)}{\varphi^{4}(q)},
$$

then

$$
x=\left(\frac{8 \sqrt{q} \psi^{2}\left(q^{2}\right) \varphi^{2}(q)}{\varphi^{4}(q)+16 q \psi^{4}\left(q^{2}\right)}\right)^{2}=\frac{4 \alpha}{(1+\alpha)^{2}},
$$

and so, by (12.27),

$$
\mu\left(x, \frac{1}{4}\right)=\frac{1}{(1+\alpha)^{\frac{1}{4}}} .
$$


By (2.1) and (5.1),

$$
\alpha=\frac{16 q \psi^{4}\left(q^{2}\right)}{\varphi^{4}(q)}=1-\frac{\varphi^{4}(-q)}{\varphi^{4}(q)}=1-\frac{\left(q ; q^{2}\right)_{\infty}^{8}}{\left(-q ; q^{2}\right)_{\infty}^{8}} .
$$

Thus, $\alpha(q)$ maps the unit interval $(0,1)$ monotonically onto itself. If we define $x$ as a function of $\alpha$ or $q$ via (12.28), then $x(\alpha)$ and hence $x(q)$ map the unit interval $(0,1)$ monotonically onto itself. Hence, given $0<x<1$,

$$
\alpha=\frac{2-x-2 \sqrt{1-x}}{x}
$$

since $0<\alpha<1$. An easy calculation gives

$$
\frac{1}{1+\alpha}=\frac{1+\sqrt{1-x}}{2}
$$

and putting this in (12.29), we deduce (12.12).

We now relate $\mu\left(x, \frac{1}{6}\right)$ in terms of radicals.

Theorem 12.3. If $0<x<1$, then

$$
\mu\left(x, \frac{1}{6}\right)=\frac{\sqrt{\sqrt{1+2 p}+\sqrt{1-p^{2}}}}{\sqrt{2}\left(1+p+p^{2}\right)^{\frac{1}{4}}},
$$

where

$$
p=\frac{-1+\sqrt{1+4 y}}{2}
$$

and $y$ is the root of the cubic equation

$$
x=\frac{27 y^{2}}{4(1+y)^{3}},
$$

which is between 0 and 2. Explicitly, $y$ is given by (12.32)

$$
\begin{array}{r}
y=\frac{1}{4 x}\left(6 \sqrt{9-8 x} \cos \left(\frac{1}{3} \arctan \left(8 \sqrt{(1-x) x^{3}}, 8 x^{2}-36 x+27\right)-\frac{2 \pi}{3}\right)\right. \\
+9-4 x) .
\end{array}
$$

Proof. Let $M(q)$ and $N(q)$ be the Eisenstein series defined at the beginning of Section 4. Let

$$
\gamma=\gamma(q)=\frac{\varphi^{4}\left(-q^{1 / 2}\right)}{\varphi^{4}\left(q^{1 / 2}\right)}
$$

and define

$$
p=p(q)=\sqrt{1-\gamma+\gamma^{2}}-\gamma .
$$

We note that both $\gamma(q)$ and $p(q)$ map the unit interval $(0,1)$ monotonically onto itself. Recall from Entries 13(i),(ii) in Chapter 17 [5, p. 126] that

$$
M\left(q^{2}\right)=\varphi^{8}(q)\left(1-\alpha+\alpha^{2}\right)
$$

and

$$
N\left(q^{2}\right)=\varphi^{12}(q)(1+\alpha)\left(1-\frac{1}{2} \alpha\right)(1-2 \alpha),
$$


where, by (5.18) and (5.19),

$$
\alpha=1-\frac{\varphi^{4}(-q)}{\varphi^{4}(q)} .
$$

Replace $q$ by $\sqrt{q}$, so that $\alpha$ is replaced by $1-\gamma$. We then deduce that

$$
M(q)=\varphi^{8}\left(q^{1 / 2}\right)\left(1-\gamma+\gamma^{2}\right)
$$

and

$$
N(q)=\frac{\varphi^{12}\left(q^{1 / 2}\right)}{2}(2-\gamma)(2 \gamma-1)(1+\gamma)
$$

From (12.34),

$$
\gamma=\frac{1-p^{2}}{1+2 p}
$$

so that

$$
\sqrt{1-\gamma+\gamma^{2}}=\frac{1+p+p^{2}}{1+2 p} .
$$

It is interesting to note that, by $(2.1)$,

$$
\frac{16 \sqrt{q} \psi^{4}(q)}{\varphi^{4}\left(q^{1 / 2}\right)}=1-\gamma=\frac{p(2+p)}{1+2 p},
$$

which gives an identification for $\alpha$ in (11.1). Thus, by (12.34)-(12.38),

$$
\begin{aligned}
x: & =\frac{M^{\frac{3}{2}}(q)-N(q)}{2 M^{\frac{3}{2}}(q)}=\frac{(\gamma-2)(2 \gamma-1)(1+\gamma)}{4\left(1-\gamma+\gamma^{2}\right)^{3 / 2}}+\frac{1}{2} \\
& =\frac{27 p^{2}(1+p)^{2}}{4\left(1+p+p^{2}\right)^{3}},
\end{aligned}
$$

which gives an identification for $\beta$ in (11.1). Hence, from Theorem 11.1, (12.40), (12.39), (12.38), (12.35), (5.18), and (5.19),

$$
\begin{aligned}
{ }_{2} F_{1}^{4}\left(\frac{1}{6}, \frac{5}{6} ; 1 ; \frac{M^{\frac{3}{2}}(q)-N(q)}{2 M^{\frac{3}{2}}(q)}\right) & =\left(1-\gamma+\gamma^{2}\right){ }_{2} F_{1}^{4}\left(\frac{1}{2}, \frac{1}{2} ; 1 ; 1-\gamma\right) \\
& =\frac{M(q)}{\varphi^{8}(\sqrt{q})} \varphi^{8}(\sqrt{q})=M(q) .
\end{aligned}
$$

We now prove that

$$
q_{6}\left(\frac{M^{\frac{3}{2}}(q)-N(q)}{2 M^{\frac{3}{2}}(q)}\right)=q,
$$

where $q_{6}(x)$ is defined by $(12.13)$ or (1.9). It is well known that [38, Chapter 5] $M(q)$ and $N(q)$ (with $q=\exp (2 \pi i \tau)$ ) are modular forms of weights 4 and 6 , respectively, and multiplier system identically equal to 1 on the full modular group. Hence, for $\operatorname{Im}(\tau)>0$,

$$
M\left(e^{-2 \pi i / \tau}\right)=\tau^{4} M\left(e^{2 \pi i \tau}\right)
$$


and

$$
N\left(e^{-2 \pi i / \tau}\right)=\tau^{6} N\left(e^{2 \pi i \tau}\right) .
$$

As in the previous proof, we set

$$
q=\exp (-2 \pi t)
$$

for $t>0$. Then (12.43) and (12.44) become

$$
M\left(e^{-2 \pi / t}\right)=t^{4} M\left(e^{-2 \pi t}\right)
$$

and

$$
N\left(e^{-2 \pi / t}\right)=-t^{6} N\left(e^{-2 \pi t}\right) .
$$

With $x$ defined by (12.40), we consider $x$ as a function of $t$. Set $z(t):=M(q)$, so that, from (12.46),

$$
z(1 / t)=t^{4} z(t)
$$

and, from (12.41),

$$
{ }_{2} F_{1}\left(\frac{1}{6}, \frac{5}{6} ; 1 ; x(t)\right)=(z(t))^{\frac{1}{4}} .
$$

By (12.40), (12.46), and (12.47),

$$
x(1 / t)=1-x(t) .
$$

Hence, from (12.2), (12.50), (12.49), and (12.48),

$$
\begin{aligned}
y & =\frac{{ }_{2} F_{1}\left(\frac{1}{6}, \frac{5}{6} ; 1 ; 1-x(t)\right)}{{ }_{2} F_{1}\left(\frac{1}{6}, \frac{5}{6} ; 1 ; x(t)\right)} \\
& =\frac{{ }_{2} F_{1}\left(\frac{1}{6}, \frac{5}{6} ; 1 ; x(1 / t)\right)}{{ }_{2} F_{1}\left(\frac{1}{6}, \frac{5}{6} ; 1 ; x(t)\right)}=\left(\frac{z(1 / t)}{z(t)}\right)^{\frac{1}{4}}=t .
\end{aligned}
$$

Therefore, by (12.13), (12.51), and (12.45),

$$
q_{6}(x(t))=\exp (-2 \pi y)=\exp (-2 \pi t)=q,
$$

which is (12.42).

It follows from (12.42), (12.41), and (12.1) that

$$
\mu\left(\frac{M^{\frac{3}{2}}(q)-N(q)}{2 M^{\frac{3}{2}}(q)}, \frac{1}{6}\right)=\frac{\varphi(q)}{(M(q))^{\frac{1}{8}}} .
$$

From Entry 25(vi) in Chapter 16 [5, p. 40],

$$
\varphi^{2}(q)=\frac{1}{2}\left(\varphi^{2}\left(q^{1 / 2}\right)+\varphi^{2}\left(-q^{1 / 2}\right)\right)
$$

and so by (12.33),

$$
\frac{\varphi^{2}(q)}{\varphi^{2}\left(q^{1 / 2}\right)}=\frac{1}{2}(1+\sqrt{\gamma}) .
$$

Hence, from (12.35) we find that

$$
M(q)=\varphi^{8}(q)\left(\frac{2}{1+\sqrt{\gamma}}\right)^{4}\left(1-\gamma+\gamma^{2}\right) .
$$


From (12.40), (12.52), (12.53), (12.37), and (12.38), we find that

$$
\begin{aligned}
\mu\left(x, \frac{1}{6}\right) & =\frac{\varphi(q)}{(M(q))^{\frac{1}{8}}}=\frac{\sqrt{\frac{1+\sqrt{\gamma}}{2}}}{\left(1-\gamma+\gamma^{2}\right)^{\frac{1}{8}}} \\
& =\frac{\sqrt{\sqrt{1+2 p}+\sqrt{1-p^{2}}}}{\sqrt{2}\left(1+p+p^{2}\right)^{\frac{1}{4}}},
\end{aligned}
$$

as claimed.

We now rewrite $(12.40)$ as

$$
x:=x(y):=\frac{27 y^{2}}{4(1+y)^{3}},
$$

where

$$
y=p(1+p) .
$$

Since $0<p<1$, we have $0<y<2$. Solving (12.55) for $p$, we deduce (12.31). We have taken the positive square root since $0<p<1$. Since (12.54) is a cubic equation in $y$, we may solve it in terms of radicals. Note that $x(-1 / 4)=x(2)=1, x(0)=0$, and

$$
\frac{d x}{d y}=-\frac{27 y(y-2)}{4(1+y)^{4}} \text {. }
$$

Thus, $x(y)$ decreases from 1 to 0 on $(-1 / 4,0)$, increases from 0 to 1 on $(0,2)$, and decreases from 1 to 0 on $(2, \infty)$. Therefore, if $0<x<1$, the equation (12.54) has three real roots, and each of the intervals $(-1 / 4,0)$, $(0,2)$, and $(2, \infty)$ contains exactly one root. We take $y$ to be the unique root that satisfies $0<y<2$. The root $y$ is given explicitly in (12.32) above.

Some consequences of our derivations of the values of $\mu(x, h)$ are some new hypergeometric transformations. From (2.13) and (2.14), we see that

$$
\alpha=\frac{16 q \psi^{4}\left(q^{2}\right)}{\varphi^{4}(q)}=\frac{(m-1)(m+3)^{3}}{16 m^{3}} .
$$

Hence, from (12.56), (12.30), (5.18), (5.19), (12.9), Lemma 2.6, and (12.8),

$$
\begin{aligned}
{ }_{2} F_{1} & \left(\frac{1}{2}, \frac{1}{2} ; 1 ; \frac{(m-1)(m+3)^{3}}{16 m^{3}}\right)={ }_{2} F_{1}\left(\frac{1}{2}, \frac{1}{2} ; 1 ; 1-\frac{\varphi^{4}(-q)}{\varphi^{4}(q)}\right) \\
& =\varphi^{2}(q)=\frac{4 m^{3 / 2}}{m^{2}+6 m-3} a(q) \\
& =\frac{4 m^{\frac{3}{2}}}{m^{2}+6 m-3}{ }_{2} F_{1}\left(\frac{1}{3}, \frac{2}{3} ; 1 ; \frac{27(m+1)^{3}\left(m^{2}-1\right)}{\left(m^{2}+6 m-3\right)^{3}}\right) .
\end{aligned}
$$

This transformation is remindful of Theorem 5.6 but is a different transformation. We have found a generalization of (12.57) via MAPLE, namely, (12.58)

$$
\begin{aligned}
& { }_{2} F_{1}\left(3 d, 2 d+\frac{1}{6} ; d+\frac{5}{6} ; \frac{(m-1)(m+3)^{3}}{16 m^{3}}\right) \\
& \quad=\left(\frac{4 m^{\frac{3}{2}}}{m^{2}+6 m-3}\right)^{6 d}{ }_{2} F_{1}\left(2 d, 2 d+\frac{1}{3} ; d+\frac{5}{6} ; \frac{27(m+1)^{3}\left(m^{2}-1\right)}{\left(m^{2}+6 m-3\right)^{3}}\right) .
\end{aligned}
$$


We omit the details. We have also found a generalization of Theorem 5.6 via MAPLE, viz.,

$$
\begin{aligned}
& { }_{2} F_{1}\left(3 d, d+\frac{1}{3} ; 2 d+\frac{2}{3} ; \frac{p^{3}(2+p)}{1+2 p}\right) \\
& \quad=\left(\frac{1+2 p}{\left(1+p+p^{2}\right)^{2}}\right)^{3 d}{ }_{2} F_{1}\left(2 d, 2 d+\frac{1}{3} ; 3 d+\frac{1}{2} ; \frac{27 p^{2}(1+p)^{2}}{4\left(1+p+p^{2}\right)^{3}}\right) .
\end{aligned}
$$

We have investigated the connection between (12.57) and Theorem 5.6 and found that (12.57) follows from Theorem 5.6 in the following way. Replace $m$ by $1+2 p$, so that $(12.57)$ can be rewritten as $(12.60)$

$$
{ }_{2} F_{1}\left(\frac{1}{2}, \frac{1}{2} ; 1 ; \frac{p(2+p)^{3}}{(1+2 p)^{3}}\right)=\frac{(1+2 p)^{\frac{3}{2}}}{1+4 p+p^{2}}{ }_{2} F_{1}\left(\frac{1}{3}, \frac{2}{3} ; 1 ; \frac{27 p(1+p)^{4}}{2\left(1+4 p+p^{2}\right)^{3}}\right) \text {. }
$$

Also, replace $p$ by $\frac{2\left(1+p+p^{2}\right)}{\left(2+2 p-p^{2}\right)}-1$ in Theorem 6.1 to obtain the identity

$$
\begin{aligned}
2(1+ & \left.p+p^{2}\right){ }_{2} F_{1}\left(\frac{1}{3}, \frac{2}{3} ; 1 ; \frac{27 p^{4}(1+p)}{2\left(2+2 p-p^{2}\right)^{3}}\right) \\
& =\left(2+2 p-p^{2}\right){ }_{2} F_{1}\left(\frac{1}{3}, \frac{2}{3} ; 1 ; \frac{27 p^{2}(1+p)^{2}}{4\left(1+p+p^{2}\right)^{3}}\right) .
\end{aligned}
$$

We now indicate the steps that lead from Theorem 5.6 to (12.60). First, apply Entry 6(i) in Chapter 17 of the second notebook [5, p. 238], i.e.,

$$
{ }_{2} F_{1}\left(\frac{1}{2}, \frac{1}{2} ; 1 ; \frac{p(2+p)^{3}}{(1+2 p)^{3}}\right)=(1+2 p){ }_{2} F_{1}\left(\frac{1}{2}, \frac{1}{2} ; 1 ; \frac{p^{3}(2+p)}{1+2 p}\right),
$$

to the left side of (12.60). Secondly, apply Theorem 5.6. Thirdly, invoke (12.61). Lastly, after employing Theorem 6.4, we deduce (12.60).

It is interesting to note that (12.61) and Theorem 5.6 give the transformation

$$
\begin{aligned}
(2+ & \left.2 p-p^{2}\right)_{2} F_{1}\left(\frac{1}{2}, \frac{1}{2} ; 1 ; \frac{p^{3}(2+p)}{1+2 p}\right) \\
& =2 \sqrt{1+2 p}{ }_{2} F_{1}\left(\frac{1}{3}, \frac{2}{3} ; 1 ; \frac{27 p^{4}(1+p)}{2\left(2+2 p-p^{2}\right)^{3}}\right) .
\end{aligned}
$$

We have found a generalization of (12.62) via MAPLE that is different from (12.58) and (12.59), namely,

$$
\begin{aligned}
& { }_{2} F_{1}\left(3 d, 2 d+\frac{1}{6} ; 4 d+\frac{1}{3} ; \frac{p^{3}(2+p)}{1+2 p}\right) \\
& \quad=\left(\frac{4(1+2 p)}{\left(2+2 p-p^{2}\right)^{2}}\right)^{3 d}{ }_{2} F_{1}\left(2 d, 2 d+\frac{1}{3} ; 3 d+\frac{1}{2} ; \frac{27 p^{4}(1+p)}{2\left(2+2 p-p^{2}\right)^{3}}\right) .
\end{aligned}
$$

Using Theorems 4.2 and 4.3 in (12.41) and then employing Theorem 2.10, we find that

$$
(1+8 x)_{2} F_{1}^{4}\left(\frac{1}{3}, \frac{2}{3} ; 1 ; x\right)={ }_{2} F_{1}^{4}\left(\frac{1}{6}, \frac{5}{6} ; 1 ; \frac{1}{2}-\frac{\left(1-20 x-8 x^{2}\right)}{2(1+8 x)^{\frac{3}{2}}}\right) .
$$


On replacing $x$ by $\frac{x^{2}-1}{8}$ we find that (12.64)

$$
{ }_{2} F_{1}\left(\frac{1}{6}, \frac{5}{6} ; 1 ; \frac{(x-1)(x+3)^{3}}{16 x^{3}}\right)=\sqrt{x}_{2} F_{1}\left(\frac{1}{3}, \frac{2}{3} ; 1 ; \frac{(x-1)(x+1)}{8}\right) .
$$

We have found a generalization via MAPLE, namely,

$$
\begin{aligned}
& { }_{2} F_{1}\left(d, d+\frac{2}{3} ; d+\frac{5}{6} ; \frac{(x-1)(x+3)^{3}}{16 x^{3}}\right) \\
& \quad=x^{3 d}{ }_{2} F_{1}\left(2 d, 2 d+\frac{1}{3} ; d+\frac{5}{6} ; \frac{(x-1)(x+1)}{8}\right) .
\end{aligned}
$$

Equation (12.64) has an elegant $q$-version; if we replace $x$ by $m$ in (12.64) and use (12.56) and Lemma 5.5, we find that

$$
\varphi\left(q^{3}\right){ }_{2} F_{1}\left(\frac{1}{6}, \frac{5}{6} ; 1 ; \frac{16 q \psi^{4}\left(q^{2}\right)}{\varphi^{4}(q)}\right)=\varphi(q){ }_{2} F_{1}\left(\frac{1}{3}, \frac{2}{3} ; 1 ;-\frac{c^{3}\left(q^{2}\right)}{c^{3}(-q)}\right)
$$

\section{CONCLUding REMARKS}

It seems inconceivable that Ramanujan could have developed the theory of signature 3 without being aware of the cubic theta function identity (2.5), and in Lemma 2.1 and Theorem 2.2 we showed how (2.5) follows from results of Ramanujan. Heng Huat Chan [19] has found a much shorter proof of (2.5) based upon results found in Ramanujan's notebooks.

H.M. Farkas and I. Kra [22, p. 124] have discovered two cubic theta-function identities different from that found by the Borweins. Let $\omega=\exp (2 \pi i / 6)$. Then, in Ramanujan's notation,

$$
\omega^{2} f^{3}\left(\omega q^{\frac{1}{3}}, \bar{\omega} q^{\frac{2}{3}}\right)+f^{3}\left(\omega q^{\frac{2}{3}}, \bar{\omega} q^{\frac{1}{3}}\right)=\omega f^{3}\left(-q^{\frac{1}{3}},-q^{\frac{2}{3}}\right)
$$

and

$$
f^{3}\left(\omega q^{\frac{2}{3}}, \bar{\omega} q^{\frac{1}{3}}\right)-f^{3}\left(\omega q^{\frac{1}{3}}, \bar{\omega} q^{\frac{2}{3}}\right)=q^{\frac{1}{3}} f^{3}(\omega q, \bar{\omega}) .
$$

Farkas and Kopeliovich [23] have generalized this to a $p$-th order identity. Garvan [27] has recently found elementary proofs of the cubic identity and the $p$-th order identities, and has found more general relations.

Almost all of the results on pages 257-262 in Ramanujan's second notebook devoted to his alternative theories are found in the first notebook, but they are scattered. In particular, they can be found on pages $96,162,204,210,212,214$, $216,218,220,242,300,301,310$, and 328 of the first notebook. Moreover, Theorem 9.11 is only found in the first notebook.

In Section 8, we crucially used properties of $b(z, q)$, a two variable analogue of $b(q)$. The theory of two variable analogues of $a(q), b(q)$, and $c(q)$ has been extensively developed by M. Hirschhorn, Garvan, and J.M. Borwein [31] and by Bhargava [11].

Some of Ramanujan's formulas for Eisenstein series in this paper were also established by K. Venkatachaliengar [41].

In [26], one of us describes how the computer algebra package MAPLE was used to understand, prove, and generalize some of the results in this paper.

Small portions of the material in this paper have been described in expository lectures by Berndt [7] and Garvan [25]. 


\section{REFERENCES}

1. A. O. L. Atkin and P. Swinnerton-Dyer, Some properties of partitions, Proc. London Math. Soc. (3) 4 (1954), 84-106.

2. W.N. Bailey, Generalized hypergeometric series, Stechert-Hafner, New York, 1964.

3. B.C. Berndt, Ramanujan's notebooks, Part I, Springer-Verlag, New York, 1985.

4. __ Ramanujan's notebooks, Part II, Springer-Verlag, New York, 1989.

5. __ Ramanujan's notebooks, Part III, Springer-Verlag, New York, 1991.

6. $\ldots$ On a certain theta-function in a letter of Ramanujan from Fitzroy House, Ganita $\mathbf{4 3}$ (1992), 33-43.

7. __ Ramanujan's theory of theta-functions, Theta Functions, From the Classical to the Modern (M. Ram Murty, ed.), Centre de Recherches Mathématiques Proceedings and Lecture Notes, Amer. Math. Soc., Providence, RI, 1993, pp. 1-63.

8. __ Ramanujan's Notebooks, Part IV, Springer-Verlag, New York, 1994.

9. B. C. Berndt and S. Bhargava, Ramanujan's inversion formulas for the lemniscate and allied functions, J. Math. Anal. Appl. 160 (1991), 504-524.

10. B.C. Berndt and L.-C. Zhang, Ramanujan's identities for eta-functions, Math. Ann. 292 (1992), 561-573.

11. S. Bhargava, On unification of the cubic analogues of the Jacobian theta-function, J. Math. Anal. Appl. 193 (1955), 543-558.

12. J.M. Borwein and P.B. Borwein, Pi and the AGM, Wiley, New York, 1987.

13. __ Explicit Ramanujan-type approximations to pi of high order, Proc. Indian Acad. Sci. (Math. Sci.) 97 (1987), 53-59.

14. _ Ramanujan's rational and algebraic series for $1 / \pi$, Indian J. Math. 51 (1987), 147-160.

15. _ More Ramanujan-type series for $1 / \pi$, Ramanujan Revisited, Academic Press, Boston, 1988, pp. 359-374.

16. _ A cubic counterpart of Jacobi's identity and the AGM, Trans. Amer. Math. Soc. 323 (1991), 691-701.

17. _ Class number three Ramanujan type series for $1 / \pi$, J. Comput. Appl. Math. 46 (1993), 281-290.

18. J.M. Borwein, P.B. Borwein, and F.G. Garvan, Some cubic modular identities of Ramanujan, Trans. Amer. Math. Soc. 343 (1994), 35-47.

19. H.H. Chan, On Ramanujan's cubic continued fraction, Acta Arith. (to appear).

20. D.V. Chudnovsky and G.V. Chudnovsky, Approximations and complex multiplication according to Ramanujan, Ramanujan Revisited, Academic Press, Boston, 1988, pp. 375-472.

21. A. Erdélyi, editor, Higher transcendental functions, Vol. 1, McGraw-Hill, New York, 1953.

22. H. M. Farkas and I. Kra, Automorphic forms for subgroups of the modular group, Israel J. Math. 82 (1993), 87-131.

23. H. M. Farkas and Y. Kopeliovich, New theta constant identities, Israel J. Math. 82 (1993), 133-141.

24. N. J. Fine, Basic hypergeometric series and applications, Math. Surveys Monographs, vol. 27, Amer. Math. Soc., Providence, RI, 1988.

25. F. G. Garvan, Cubic modular identities of Ramanujan, hypergeometric functions and analogues of the arithmetic-geometric mean iteration, The Rademacher Legacy to Mathematics (G.E. Andrews, D.M. Bressoud, and A. Parson, eds.), Contemp. Math., vol. 166, Amer. Math. Soc., Providence, RI, 1994, pp. 245-264.

26. __ Ramanujan's theories of elliptic functions to alternatiave bases-a symbolic excursion, J. Symbolic Comput. (to appear).

27. _ A combinatorial proof of the Farkas-Kra theta function identities and their generalizations, J. Math. Anal. Appl. 195 (1995). 
28. G. Gasper and M. Rahman, Basic hypergeometric series, Cambridge Univ. Press, Cambridge, 1990.

29. E. Goursat, Sur l'équation différentielle linéaire qui admet pour intégrale la série hypergéométrique, Ann. Sci. École Norm. Sup. (2) 10 (1881), 3-142.

30. P. Henrici, Applied and computational complex analysis, Vol. 1, Wiley, New York, 1990.

31. M. Hirschhorn, F. Garvan, and J. Borwein, Cubic analogues of the Jacobian theta function $\theta(z, q)$, Canad. J. Math. 45 (1993), 673-694.

32. M. I. Knopp, Modular functions in analytic number theory, Markham, Chicago, IL, 1970.

33. S. Ramanujan, Modular equations and approximations to $\pi$, Quart. J. Math. (Oxford) 45 (1914), 350-372.

34. __ On certain arithmetical functions, Trans. Cambridge Philos. Soc. 22 (1916), 159-184.

35. _ Notebooks (2 volumes), Tata Institute of Fundamental Research, Bombay, 1957.

36. Collected papers, Chelsea, New York, 1962.

37. _ The lost notebook and other unpublished papers, Narosa, New Delhi, 1988.

38. R.A. Rankin, Modular forms and functions, Cambridge Univ. Press, Cambridge, 1977.

39. B. Schoeneberg, Elliptic modular functions, Springer-Verlag, Berlin, 1974.

40. L.-C. Shen, On Ramanujan's theory of elliptic functions based on the hypergeometric series ${ }_{2} F_{1}\left(\frac{1}{3}, \frac{2}{3} ; 1 ; x\right)$, J. Number Theory (to appear).

41. K. Venkatachaliengar, Development of elliptic functions according to Ramanujan, Tech. Report 2, Madurai Kamaraj University, Madurai, 1988.

42. E.T. Whittaker and G.N. Watson, $A$ course of modern analysis, 4th ed., Cambridge Univ. Press, Cambridge, 1966.

Department of Mathematics, University of Illinois, 1409 West Green St., Urbana, ILLINOIS 61801

Department of Mathematics, University of Mysore, Manasa Gangotri, Mysore 570006, INDIA

Department of Mathematics, University of Florida, Gainesville, Florida 32611 